\title{
Top-quark pair production close to threshold: Top-quark mass, width, and momentum distribution
}

\author{
A. H. Hoang \\ Theory Division, CERN, CH-1211 Geneva 23, Switzerland \\ T. Teubner \\ Deutsches Elektronen-Synchrotron DESY, D-22603 Hamburg, Germany \\ (Received 27 April 1999; published 9 November 1999)
}

\begin{abstract}
The complete next-to-next-to-leading order (NNLO) QCD corrections to the total cross section $\sigma\left(e^{+} e^{-}\right.$ $\left.\rightarrow Z^{*}, \gamma^{*} \rightarrow t \bar{t}\right)$, in the kinematic region close to the top-quark-top-antiquark threshold, are calculated by solving the corresponding Schrödinger equations exactly in momentum space in a consistent momentum cutoff regularization scheme. The corrections coming from the same NNLO QCD effects to the top-quark threemomentum distribution $d \sigma / d\left|\vec{k}_{t}\right|$ are determined. We discuss the origin of the large NNLO corrections to the peak position and the normalization of the total cross section observed in previous works and propose a new top-quark mass definition, the $1 S$ mass $M_{1 S}$, which stabilizes the peak in the total cross section. If the influence of beamstrahlung and initial state radiation on the mass determination is small, a theoretical uncertainty on the $1 S$ top-quark mass measurement of $200 \mathrm{MeV}$ from the total cross section at the linear collider seems possible. We discuss how well the $1 S$ mass can be related to the $\overline{\mathrm{MS}}$ mass. We propose a consistent way to implement the top-quark width at NNLO by including electroweak effects into the NRQCD matching coefficients, which can then become complex. [S0556-2821(99)07219-7]
\end{abstract}

PACS number(s): 14.65.Ha, 12.38.Bx, 13.85.Lg

\section{INTRODUCTION}

It will be one of the primary goals of a future $e^{+} e^{-}$linear collider (LC) or $\mu^{+} \mu^{-}$pair collider (FMC) to measure and determine the properties of the top quark, whose existence has been confirmed at the Fermilab Tevatron $\left(M_{t}=173.8 \pm 5\right.$ $\mathrm{GeV}[1])$. Although the top quark will also be the object of intense studies at run II at the Tevatron and at the CERN Large Hadron Collider (LHC), the measurements at a LC are important to fill the gaps left by the measurements in the environment of the hadron colliders. One of the most dramatic improvements attainable at a LC can be expected in the determination of the top-quark mass. At the LHC, where the mass is extracted from the peak in the top-quark invariant mass spectrum of the $W$ and $b$ originating from the top-quark decay, a final (systematics-dominated) top-quark mass uncertainty at the level of $2-3 \mathrm{GeV}$ seems realistic. More precision will be difficult owing to unavoidable conceptual and practical problems and ambiguities in disentangling the top quark invariant mass from numerous effects in the environment of hadron colliders. At a LC the top-quark mass can be determined from a measurement of the line shape of the total cross section $\sigma\left(e^{+} e^{-} \rightarrow Z^{*}, \gamma^{*} \rightarrow t \bar{t}\right)$ for center-of-mass energies around the threshold, $\sqrt{q^{2}} \approx 350 \mathrm{GeV}$. The rise of the cross section with increasing center-of-mass energy is directly correlated to the mass of the top quark. Because the total cross section describes the rate of color singlet topquark-top-antiquark events, it is theoretically and practically much better under control than the top-quark invariant mass distribution. Because of the large top-quark width $[\Gamma(t$ $\left.\rightarrow b W)=\left(G_{F} / \sqrt{2}\right)\left(M_{t}^{3} / 8 \pi\right) \approx 1.5 \mathrm{GeV}\right]$ the top-quark-top- antiquark pair cannot hadronize into toponium resonances, and the cross section represents a smooth line shape showing only a moderate peaklike enhancement, which is the broad remnant of the $1 S$ resonance. At the same time the top-quark width effectively serves as an infrared cutoff [2] and as a natural smearing mechanism [3], which allows us to calculate the cross section in the threshold region to high precision using perturbative QCD. It is therefore possible to reliably relate the cross section line shape to the parameters of the standard model, most notably the top-quark mass and the strong coupling. LC simulation studies have demonstrated that for $50-100 \mathrm{fb}^{-1}$ total integrated luminosity an experimental uncertainty of order $100-200 \mathrm{MeV}$ can be expected in the top-quark mass determination from a line shape scan of the total cross section [4]. Evidently, at this level of precision an adequate control over theoretical uncertainties has to be achieved. In particular, a precise definition of the topquark mass extracted from the experiment has to be given.

For center-of-mass energies close to the top-quark-topantiquark threshold, the top quarks are produced with nonrelativistic velocities $\mathrm{V} \ll 1$. Therefore the relevant physical scales, which govern the top-quark-top-antiquark dynamics, the top-quark mass $M_{t}$, the relative momentum $M_{t} \vee$ and the top-quark kinetic energy $M_{t} \mathrm{v}^{2}$, are widely separated. Because ratios of the three scales arise, the cross section close to threshold cannot be calculated using the standard multiloop expansion in the strong coupling $\alpha_{s}$, but rather a double expansion in $\alpha_{s}$ and $\mathrm{v}$. In the nonrelativistic limit the most prominent indication of this feature is known as the "Coulomb singularity," which originates from the ratio $M_{t} /\left(M_{t} \mathrm{v}\right)$. The Coulomb singularity is visible as a singular 
$\left(\alpha_{s} / \mathrm{v}\right)^{n}$ behavior in the $n$-loop QCD correction to the amplitude $\gamma \rightarrow t \bar{t}$ for $\mathrm{v} \rightarrow 0$. The most economic and systematic way to tackle this problem is to employ the concept of effective theories by using the hierarchy $M_{t} \gg M_{t} \mathrm{v} \gg M_{t} \mathrm{v}^{2}$ $>\Gamma_{t} \gg \Lambda_{\mathrm{QCD}}$ and by successively integrating out higher momentum effects. At leading order (LO) and next-to-leading order (NLO) in the nonrelativistic expansion, ${ }^{1}$ the use of effective field theoretical methods seems not to be vital, because, clearly, in the first approximation the top-quark-topantiquark pair can be described by a nonrelativistic Schrödinger equation [2,5] and because, luckily, the relevant current operators do not have any anomalous dimension at the one-loop level. Beyond NLO, however, anomalous dimensions arise when relativistic effects suppressed by $v^{2}$ are included. This makes the use of effective field theoretical methods mandatory. In addition, the effective field theoretical approach allows for the development of a power counting scheme, which allows for a systematic identification of all effects contributing to a certain order of approximation. Those power counting rules in fact confirm that at next-tonext-to-leading order (NNLO) the top-quark-top-antiquark pair can be completely described by a conventional Schrödinger equation containing an instantaneous potential. In general, the same conclusions cannot be drawn for nonrelativistic bottom-quark-bottom-antiquark or charm-anticharm systems.

A large number of theoretical studies at LO and NLO [6-10] have been carried out in the past in order to study the feasibility of the threshold scan and other measurements at the top-quark-top-antiquark threshold. Recently, first NNLO QCD calculations for the total vector-current-induced cross section $\sigma\left(e^{+} e^{-} \rightarrow \gamma^{*} \rightarrow t \bar{t}\right)$ have been performed in Refs. [11-13]. These analyses were based on nonrelativistic quantum chromodynamics (NRQCD) $[14,15]$ and on the direct matching procedure $[16,17]$. In this work we extend the calculations and also determine the NNLO QCD relativistic corrections to the top quark three-momentum distribution. Interconnection effects caused by gluon exchange among top-quark-top-antiquark decay and production processes are not considered in this work. They are known to vanish at NLO for the total cross section [18-20], but can lead to sizeable corrections in the momentum distribution $[21,22]$. We also include the total cross section and the threemomentum distribution induced by the axial-vector current. Because the latter quantities are suppressed by $v^{2}$ with respect to the vector-current-induced ones, we only determine them at leading order in the nonrelativistic expansion. It turns out that the size of the axial-vector contributions is smaller than the theoretical uncertainties contained in the dominant vector-current-induced cross section. A discussion on the size of the axial-vector-current-induced contributions can also be found in Ref. [23]. The three-momentum distributions presented in this work represent a first step towards

\footnotetext{
${ }^{1}$ We will define what is meant by LO, NLO, NNLO, etc., in the framework of the nonrelativistic expansion at the beginning of Sec. II.
}

an exclusive treatment of the top-quark-top-antiquark final state at NNLO close to threshold. We analyze in detail the origin of the large NNLO corrections to the peak position and the normalization in the total cross section already observed in Refs. [11-13], and show that the instabilities in the peak position are a consequence of the use of the pole mass scheme. We show that the pole mass parameter is irrelevant to the peak position and define a new top quark mass, the $1 S$ mass, which is more suitable to parametrize the total cross section. Whereas the $1 S$ mass leads to a considerable stabilization of the peak position it does not affect the large corrections to the normalization. In this paper we also propose a NNLO generalization of the energy replacement rule " $E$ $\rightarrow E+i \Gamma_{t}$,' ' by Fadin and Khoze, for the implementation of the top-quark width by including electroweak corrections into the matching conditions of NRQCD. In general this leads to NRQCD short-distance coefficients that have an imaginary part.

The program of this paper is as follows: in Sec. II we review the conceptual framework of the effective theories NRQCD and potential (P)NRQCD as far as it is relevant to the NNLO calculations carried out in this work. Section III contains a derivation of the integral equations that have to be solved and Sec. IV describes our cutoff regularization scheme. In Secs. II-IV the top-quark width is neglected. In Sec. V we discuss the effects of the top-quark width from the point of view of (P)NRQCD. Some details about our numerical methods to solve the integral equations are given in Sec. VI. A first analysis of the total cross section and the three momentum distribution in the pole mass scheme is given in Sec. VII. Section VIII concentrates on the origin and interpretation of the large NNLO corrections in the pole mass scheme and introduces the $1 S$ mass. The relation of the $1 S$ mass to other mass definitions is discussed. Section IX contains our conclusions. In the Appendix, details of the NNLO matching calculation are given in the framework of our regularization scheme.

\section{THE CONCEPTUAL FRAMEWORK-NRQCD AND PNRQCD}

In this section we review the effective field theories NRQCD and PNRQCD, which form the conceptual framework in which the NNLO corrections to the top-quark-topantiquark cross section close to threshold are calculated. By $\mathrm{N}^{k} \mathrm{LO}(k=0,1,2, \ldots)$ for the total cross section we mean a resummation of all terms proportional to $\alpha_{s}^{m} \mathrm{v}^{n}$, with $m+n$ $=1, \ldots, k+1$, in perturbation theory in $\alpha_{s}$ supplemented by a subsequent expansion in the top-quark velocity, i.e., in the limit $\alpha_{s} \ll \mathrm{V} \ll 1$. Thus at the NNLO level all terms proportional to $\mathrm{v} \Sigma_{n=0}^{\infty}\left(\alpha_{s} / \mathrm{v}\right)^{n}\left[1 ; \alpha_{s}, \mathrm{v} ; \alpha_{s}^{2}, \alpha_{s} \mathrm{v}, \mathrm{v}^{2}\right]$ have to be resummed to all orders in conventional perturbation theory in $\alpha_{s}$, where the dominant terms in the nonrelativistic limit are determined by a nonrelativistic Schrödinger equation with a Coulomb potential $V(\boldsymbol{r})=-C_{F} \alpha_{s} / r, C_{F}=4 / 3$. In this context one has to count the strong coupling $\alpha_{s}$ of order $\mathrm{v}$, as long as the renormalization scale is much larger than the typical hadronization scale $\Lambda_{\mathrm{QCD}}$. For simplicity we postpone the effects of the top-quark width until Sec. V. 
NRQCD $[14,15]$ is an effective field theory of QCD specifically designed to handle nonrelativistic heavy quarkantiquark systems. NRQCD is based on the separation of the low momentum scales $M_{t} \vee$ and $M_{t} \mathrm{v}^{2}$, which govern the nonrelativistic quark-antiquark dynamics, from the high momentum scale $M_{t}$, which is relevant for hard effects involved in the quark-antiquark production process and quarkantiquark and quark-gluon interactions. The NRQCD Lagrangian for the top-quark-top-antiquark system is obtained from QCD by integrating out all hard quark and gluon momenta of order $M_{t}$ or larger, and the corresponding antiparticle poles of the small components. Treating all quarks except the top quark as massless, the resulting nonrenormalizable Lagrangian reads

$$
\begin{aligned}
\mathcal{L}_{\mathrm{NRQCD}}= & -\frac{1}{2} \operatorname{Tr} G^{\mu \nu} G_{\mu \nu}+\sum_{q=u, d, s, c, b} \bar{q} i \not D q \\
& +\psi^{\dagger}\left[i D_{t}+c_{2} \frac{\boldsymbol{D}^{2}}{2 M_{t}}+c_{4} \frac{\boldsymbol{D}^{4}}{8 M_{t}^{3}}+\cdots+\frac{c_{F} g_{s}}{2 M_{t}} \boldsymbol{\sigma} \cdot \boldsymbol{B}\right. \\
& +\frac{c_{\mathbf{D}} g_{s}}{8 M_{t}^{2}}(\boldsymbol{D} \cdot \boldsymbol{E}-\boldsymbol{E} \cdot \boldsymbol{D}) \\
& \left.+\frac{c_{S} g_{s}}{8 M_{t}^{2}} i \sigma(\boldsymbol{D} \times \boldsymbol{E}-\boldsymbol{E} \times \boldsymbol{D})+\cdots\right] \psi \\
& +\chi^{\dagger}\left[i D_{t}-c_{2} \frac{\boldsymbol{D}^{2}}{2 M_{t}}-c_{4} \frac{\boldsymbol{D}^{4}}{8 M_{t}^{3}}+\cdots-\frac{c_{F} g_{s}}{2 M_{t}} \boldsymbol{\sigma} \cdot \boldsymbol{B}\right. \\
& +\frac{c_{D} g_{s}}{8 M_{t}^{2}}(\boldsymbol{D} \cdot \boldsymbol{E}-\boldsymbol{E} \cdot \boldsymbol{D}) \\
& \left.+\frac{c_{S} g_{s}}{8 M_{t}^{2}} i \sigma(\boldsymbol{D} \times \boldsymbol{E}-\boldsymbol{E} \times \boldsymbol{D})+\cdots\right] \chi,
\end{aligned}
$$

where only those terms are displayed explicitly which are relevant to the NNLO calculations in this work. The gluonic and light quark degrees of freedom are described by the conventional relativistic Lagrangian, where $G^{\mu \nu}$ is the gluon strength field tensor, $q$ the Dirac spinor of a massless quark. The nonrelativistic top quark and antitop quark are described by the Pauli spinors $\psi$ and $\chi$, respectively. For convenience all color indices are suppressed and summations over color indices are understood. $D_{t}$ and $\boldsymbol{D}$ are the time and space components of the gauge covariant derivative $D_{\mu}$, and $E^{i}$ $=G^{0 i}$ and $B^{i}=\frac{1}{2} \epsilon^{i j k} G^{j k}$ the electric and magnetic components of the gluon field strength tensor. The short-distance coefficients $c_{2}, c_{4}, c_{F}, c_{D}, c_{S}$ are normalized to one at the Born level. The subscripts $F, D$, and $S$ stand for Fermi, Darwin, and spin-orbit. We emphasize that the mass parameter $M_{t}$ used for the formulation of NRQCD is the top-quark pole mass. Although it is known that this choice can lead to a bad behavior of the perturbative coefficients at large orders, the pole mass is still the most convenient mass parameter to be used at this stage, because the formulation of NRQCD is particularly simple in this scheme.
In addition to integrating out hard quark and gluon momenta and the small components in the QCD Lagrangian one also has to do the same in the vector $\left(j_{\mu}^{\vee}=\bar{t} \gamma_{\mu} t\right)$ and the axial-vector currents $\left(j_{\mu}^{a}=\bar{t} \gamma_{\mu} \gamma_{5} t\right)$, which produce and annihilate the top-quark-top-antiquark pair close to the threshold with center-of-mass energy $\sqrt{q^{2}}$. This means that we have to expand the respective QCD currents in terms of NRQCD currents carrying the proper quantum numbers. In momentum space representation the expansion of the QCD vector current in terms of ${ }^{3} S_{1}$ NRQCD currents reads $(k$ $=1,2,3)$

$$
\begin{gathered}
\widetilde{j}_{k}^{\vee}(q)=c_{1}^{\vee}\left(\tilde{\psi}^{\dagger} \sigma_{k} \tilde{\chi}\right)(q)-\frac{c_{2}^{\vee}}{6 M_{t}^{2}}\left[\tilde{\psi}^{\dagger} \sigma_{k}\left(-\frac{i}{2} \overleftrightarrow{\boldsymbol{D}}\right)^{2} \tilde{\chi}\right](q)+\cdots, \\
\widetilde{j}_{k}^{\vee}(-q)=c_{1}^{\vee}\left(\tilde{\chi}^{\dagger} \sigma_{k} \widetilde{\psi}\right)(-q)-\frac{c_{2}^{\vee}}{6 M_{t}^{2}}\left[\tilde{\chi}^{\dagger} \sigma_{k}\left(-\frac{i}{2} \overleftrightarrow{\boldsymbol{D}}\right)^{2} \widetilde{\psi}\right](-q) \\
+\cdots,
\end{gathered}
$$

and the expansion of the QCD axial-vector current in terms of ${ }^{3} P_{1}$ NRQCD currents

$$
\begin{gathered}
\widetilde{j}_{k}^{a}(q)=\frac{c_{1}^{a}}{M_{t}}\left[\widetilde{\psi}^{\dagger}\left(-\frac{i}{2} \overleftrightarrow{\boldsymbol{D}} \times \boldsymbol{\sigma}\right)_{k} \tilde{\chi}\right](q)+\cdots, \\
\widetilde{j}_{k}^{a}(-q)=\frac{c_{1}^{a}}{M_{t}}\left[\tilde{\chi}^{\dagger}\left(-\frac{i}{2} \overleftrightarrow{\boldsymbol{D}} \times \boldsymbol{\sigma}\right)_{k} \widetilde{\psi}\right](-q)+\cdots,
\end{gathered}
$$

where the constants $c_{1,2}^{\vee}$ and $c_{1}^{a}$ are the short-distance coefficients normalized to one at the Born level. The time components of the currents do not contribute because the trace over the massless lepton fields that describe the $e^{+} e^{-}$annihilation process is proportional to $\left(\delta^{i j}-e^{i} e^{j}\right),\left(e^{1}, e^{2}, e^{3}\right)$ being the unit-vector pointing into the center-of-mass electron direction. In addition, the zero component of the vector current vanishes. The dominant NRQCD current in the expansion of the QCD vector current has dimension 3. Thus for a NNLO description of the cross section we have to expand the QCD vector current in NRQCD currents up to dimension 5. The QCD axial-vector current only needs to be expanded up to dimension 4. For the NNLO calculation of the cross section only the $\mathcal{O}\left(\alpha_{s}^{2}\right)$ short-distance corrections to the coefficient $c_{1}^{v}$ have to be calculated.

To formulate the total $t \bar{t}$ production cross sections in $e^{+} e^{-}$annihilation in the nonrelativistic region at NNLO in NRQCD, we first define the vector and axial-vector-currentinduced cross sections using the optical theorem and starting from their corresponding expressions in full QCD:

$$
\begin{aligned}
R^{\vee}\left(q^{2}\right) & =\frac{4 \pi}{q^{2}} \operatorname{Im}\left[-i \int d^{4} x e^{i q \cdot x}\left\langle 0\left|T j_{i}^{\vee}(x) j^{\vee i}(0)\right| 0\right\rangle\right] \\
& \equiv \frac{4 \pi}{q^{2}} \operatorname{Im}\left[-i\left\langle 0\left|T \widetilde{j}_{i}^{\vee}(q) \widetilde{j}^{\vee i}(-q)\right| 0\right\rangle\right],
\end{aligned}
$$




$$
\begin{aligned}
R^{a}\left(q^{2}\right) & =\frac{4 \pi}{q^{2}} \operatorname{Im}\left[-i \int d^{4} x e^{i q \cdot x}\left\langle 0\left|T j_{i}^{a}(x) j^{a i}(0)\right| 0\right\rangle\right] \\
& \equiv \frac{4 \pi}{q^{2}} \operatorname{Im}\left[-i\left\langle 0\left|T \widetilde{j}_{i}^{a}(q) \widetilde{j}^{a i}(-q)\right| 0\right\rangle\right] .
\end{aligned}
$$

In terms of $R^{\vee}$ and $R^{a}$ the total cross section $\sigma_{\text {tot }}^{\gamma, Z}\left(e^{+} e^{-}\right.$ $\left.\rightarrow \gamma^{*}, Z^{*} \rightarrow t \bar{t}\right)$ reads

$$
\begin{aligned}
\sigma_{\text {tot }}^{\gamma, Z}\left(q^{2}\right)= & \sigma_{p t}\left[Q_{t}^{2}-2 \frac{q^{2}}{q^{2}-M_{Z}^{2}} \mathrm{v}_{e} \mathrm{v}_{t} Q_{t}\right. \\
& \left.+\left(\frac{q^{2}}{q^{2}-M_{Z}^{2}}\right)^{2}\left[\mathrm{v}_{e}^{2}+a_{e}^{2}\right] \mathrm{v}_{t}^{2}\right] R^{\mathrm{v}}\left(q^{2}\right) \\
& +\sigma_{p t}\left(\frac{q^{2}}{q^{2}-M_{Z}^{2}}\right)^{2}\left[\mathrm{v}_{e}^{2}+a_{e}^{2}\right] a_{t}^{2} R^{a}\left(q^{2}\right),
\end{aligned}
$$

where

$$
\begin{gathered}
\sigma_{p t}=\frac{4 \pi \alpha^{2}}{3 q^{2}}, \\
\mathrm{v}_{f}=\frac{T_{3}^{f}-2 Q_{f} \sin ^{2} \theta_{W}}{2 \sin \theta_{W} \cos \theta_{W}}, \\
a_{f}=\frac{T_{3}^{f}}{2 \sin \theta_{W} \cos \theta_{W}} .
\end{gathered}
$$

Here, $\alpha$ is the fine structure constant, $Q_{t}=2 / 3$ the electric charge of the top quark, $\theta_{W}$ the Weinberg angle, and $T_{3}^{f}$ refers to the third component of the weak isospin; $Q_{t}^{2} R^{\vee}$ is equal to the total normalized photon-induced cross section, which is usually referred to as the $R$ ratio. To determine $R^{v}$ and $R^{a}$ at NNLO in NRQCD we insert the expansions in Eqs. (2)-(5) into Eqs. (6) and (7). This leads to the expressions

$$
\begin{aligned}
& R_{\mathrm{NNLO}}^{\mathrm{v} \text { thr }}\left(q^{2}\right)=\frac{4 \pi}{q^{2}} C^{\mathrm{v}} \operatorname{Im}\left[\mathcal{A}^{\mathrm{v}}\left(q^{2}\right)\right]+\cdots, \\
& R_{\mathrm{NNLO}}^{a, \mathrm{thr}}\left(q^{2}\right)=\frac{4 \pi}{q^{2}} C^{a} \operatorname{Im}\left[\mathcal{A}^{a}\left(q^{2}\right)\right]+\cdots,
\end{aligned}
$$

where

$$
\begin{aligned}
\mathcal{A}^{\vee}= & i\langle 0|\left[\widetilde{\psi}^{\dagger} \vec{\sigma} \widetilde{\chi}+\frac{1}{6 M_{t}^{2}} \widetilde{\psi}^{\dagger} \overrightarrow{\boldsymbol{\sigma}}\left(-\frac{i}{2} \overleftrightarrow{\boldsymbol{D}}\right)^{2} \tilde{\chi}\right] \\
& \times\left[\tilde{\chi}^{\dagger} \vec{\sigma} \widetilde{\psi}+\frac{1}{6 M_{t}^{2}} \widetilde{\chi}^{\dagger} \vec{\sigma}\left(-\frac{i}{2} \overleftrightarrow{\boldsymbol{D}}\right)^{2} \widetilde{\psi}\right]|0\rangle, \\
\mathcal{A}^{a}= & i\left\langle 0\left|\left[\widetilde{\psi}^{\dagger}\left(-\frac{i}{2} \overleftrightarrow{\boldsymbol{D}} \times \boldsymbol{\sigma}\right) \tilde{\chi}\right]\left[\tilde{\chi}^{\dagger}\left(-\frac{i}{2} \overleftrightarrow{\boldsymbol{D}} \times \boldsymbol{\sigma}\right) \widetilde{\psi}\right]\right| 0\right\rangle,
\end{aligned}
$$

and

$$
\begin{aligned}
& C^{\vee}=\left(c_{1}^{\vee}\right)^{2}, \\
& C^{a}=1 .
\end{aligned}
$$

The expressions for $R^{\vee}$ and $R^{a}$ at NNLO in the nonrelativistic expansion in Eqs. (12) and (13) represent an application of the factorization formalism proposed by Bodwin, Braaten, and Lepage [15]. The cross sections are written as a sum of absorptive parts of nonrelativistic current correlators, each of which is multiplied by a short-distance coefficient. The vector correlator $\mathcal{A}^{\mathrm{v}}$ describes the top-quark-top-antiquark system produced in an $S$-wave spin triplet state and the axialvector correlator $\mathcal{A}^{a}$ describes the system in a corresponding $P$-wave triplet state. The axial vector-current-induced cross section is suppressed by $v^{2}$ with respect to the vectorcurrent-induced cross section. We note that the correlators are defined within a proper regularization scheme. For convenience we have not expanded $\mathcal{A}^{\mathrm{v}}$ into a sum of a dimension 6 and a dimension 8 current correlator. The short distance coefficients $C^{\vee}$ and $C^{a}$ encode the effects of quark and gluon momenta of order the top-quark mass or larger in topquark-top-antiquark production and annihilation vertex diagrams. As the nonrelativistic correlators they are regularization-scheme-dependent. In principle the nonrelativistic correlators $\mathcal{A}^{\vee}$ and $\mathcal{A}^{a}$ could be calculated from the Feynman rules derived from the NRQCD Lagrangian (1). Such a task, however, would be quite cumbersome, because an infinite number of diagrams would still have to be resummed. Clearly, we would like to derive an equation of motion for the off-shell top-quark four point Green function, which in the nonrelativistic limit reduces to the well known Schrödinger equation, which automatically carries out the resummation of the relevant diagrams. A formal and systematic way to achieve that is to also integrate out top-quark and gluon modes carrying momenta of the order the inverse Bohr radius $\sim M_{t} \vee$. The resulting effective field theory is called "potential NRQCD" (PNRQCD) [24]. The basic ingredient to construct PNRQCD is to identify the relevant physical momentum regions in the description of heavy quarkantiquark systems in the framework of NRQCD. Those momentum regions have been identified in Ref. [25] by constructing an asymptotic expansion of Feynman diagrams describing heavy quark-antiquark production or annihilation close to threshold in terms of the heavy quark center-of-mass velocity. Because NRQCD is not Lorentz covariant, the time and the spatial components of the momenta have to be treated independently. There are momentum regions where time and spatial components are of a different order in the velocity counting. The relevant momentum regions are "soft" $\quad\left(k^{0} \sim M_{t} \vee, \vec{k} \sim M_{t} \vee\right), \quad$ "potential" $\quad\left(k^{0} \sim M_{t} \mathrm{v}^{2}, \vec{k}\right.$ $\left.\sim M_{t} \mathrm{v}\right)$, and "ultrasoft" $\left(k^{0} \sim M_{t} \mathrm{v}^{2}, \vec{k} \sim M_{t} \mathrm{v}^{2}\right)$. It can be shown that heavy quarks and gluons can have soft and potential momenta, but only gluons can have ultrasoft momenta. A momentum region with $k^{0} \sim M_{t} \vee, \vec{k} \sim M_{t} \mathrm{v}^{2}$ does not exist. It is in principle not excluded that there are momentum regions scaling as $M_{t} \mathrm{v}^{n}$ with $n>2$, but even if such regions existed they would be irrelevant to top-quark production because they would represent modes below the hadronization scale. 
To construct PNRQCD one integrates out "soft" heavy quarks and gluons $\left(k^{0} \sim M_{t} \mathrm{v}, \vec{k} \sim M_{t} \vee\right)$ and "potential" gluons $\left(k^{0} \sim M_{t} \vee^{2}, \vec{k} \sim M_{t} \vee\right)$, supplemented by an expansion in momentum components of order $M_{t} \mathrm{v}^{2}$. Heavy quarks carrying potential momenta and gluons with ultrasoft momenta are kept as dynamical fields. This leads to spatially nonlocal four (heavy) quark operators which represent a coupling of a quark-antiquark pair separated by distances of order of the Bohr radius $\sim 1 / M_{t} \vee$. For a quark-antiquark pair in a color singlet state this nonlocal interaction is nothing else than the instantaneous potential of a quark-antiquark separated by a distance of order the inverse Bohr radius. Generically the PNRQCD Lagrangian has the form

$\mathcal{L}_{\mathrm{PNRQCD}}=\tilde{L}_{\mathrm{NRQCD}}+\int d^{3} \boldsymbol{r}\left(\psi^{\dagger} \psi\right)(\boldsymbol{r}) V(\boldsymbol{r})\left(\chi^{\dagger} \chi\right)(0)$,

where the tilde above $\mathcal{L}_{\mathrm{NRQCD}}$ on the right-hand side (RHS) of Eq. (18) indicates that the corresponding operators only describe potential quark and ultrasoft gluonic degrees of freedom. In addition, an expansion in momentum components $\sim M_{t} \mathrm{v}^{2}$ is understood. $V$ is the heavy quark-antiquark potential and is given below. Using the velocity counting rules for potential heavy quarks mentioned above we see that the LO contribution to $V$, the Coulomb potential $-C_{F} \alpha_{s} /|\boldsymbol{r}|$, counts as $\mathrm{v}^{2}$, i.e., it is of the same order as the kinetic energy. Thus, as is well known, in the nonrelativistic limit, the Coulombic interaction between the heavy quark pair has to be treated exactly rather than perturbatively. We note that the expansion of the heavy quark currents in terms of NRQCD currents [Eqs. (2)-(5)] is in general also affected by going from NRQCD to PNRQCD, because also in the NRQCD currents soft and potential gluonic degrees of freedom have to be integrated out. However, at NNLO this does not affect the results displayed in Eqs. (2) and (5). The only (and fortunate) practical consequence is that we can neglect the gluonic contribution in the covariant derivatives. Collecting all terms from the PNRQCD Lagrangian which contribute at NNLO, i.e., count as $\mathrm{v}^{2}, \mathrm{v}^{3}$, or $\mathrm{v}^{4}$, one can derive the following equation of motion in momentum space representation for the Green function of the time-independent Schrödinger equation, valid at NNLO in the nonrelativistic expansion

$$
\begin{aligned}
& {\left[\frac{\boldsymbol{k}^{2}}{M_{t}}-\frac{\boldsymbol{k}^{4}}{4 M_{t}^{3}}-\left(\frac{p_{0}^{2}}{M_{t}}-\frac{p_{0}^{4}}{4 M_{t}^{3}}\right)\right] \tilde{G}\left(\boldsymbol{k}, \boldsymbol{k}^{\prime} ; q^{2}\right)} \\
& \quad+\int \frac{d^{3} \boldsymbol{p}^{\prime}}{(2 \pi)^{3}} \tilde{V}\left(\boldsymbol{k}, \boldsymbol{p}^{\prime}\right) \tilde{G}\left(\boldsymbol{p}^{\prime}, \boldsymbol{k}^{\prime} ; q^{2}\right)=(2 \pi)^{3} \delta^{(3)}\left(\boldsymbol{k}-\boldsymbol{k}^{\prime}\right),
\end{aligned}
$$

where

$$
\widetilde{V}\left(\boldsymbol{k}, \boldsymbol{k}^{\prime}\right)=\widetilde{V}_{c}\left(\boldsymbol{k}-\boldsymbol{k}^{\prime}\right)+\widetilde{V}_{\mathrm{BF}}\left(\boldsymbol{k}, \boldsymbol{k}^{\prime}\right)+\widetilde{V}_{\mathrm{NA}}\left(\boldsymbol{k}-\boldsymbol{k}^{\prime}\right)
$$

and

$$
p_{0}^{2}=\frac{q^{2}}{4}-M_{t}^{2}
$$

The parameter $p_{0}$ is equal to the center-of-mass threemomentum of the top quarks. We have chosen this rather unusual representation for the energy parameter since it greatly simplifies, because of its symmetric form in Eq. (19), the analytic matching calculations we carry out in the Appendix. The same trick has already been used in Ref. [17] and later in Refs. [11-13]. It is a nontrivial fact that the PNRQCD operators, which describe interactions of the heavy quarks with ultrasoft gluons, do not contribute at NNLO, and that a conventional two-body Schrödinger equation with an instantaneous potential is fully capable of resumming all terms $\propto \alpha_{s}^{m} \mathrm{v}^{n}$ that belong to NNLO. We come back to this issue at the end of this section.

The individual potentials in momentum space representation read $\left(a_{s} \equiv \alpha_{s}(\mu), C_{A}=3, C_{F}=4 / 3, T=1 / 2, Q \equiv \boldsymbol{k}-\boldsymbol{k}^{\prime}\right)$ :

$$
\begin{aligned}
\tilde{V}_{c}(\boldsymbol{k})= & -\frac{4 \pi C_{F} a_{s}}{\boldsymbol{k}^{2}}\left\{1+\left(\frac{a_{s}}{4 \pi}\right)\left[-\beta_{0} \ln \left(\frac{\boldsymbol{k}^{2}}{\mu^{2}}\right)+a_{1}\right]+\left(\frac{a_{s}}{4 \pi}\right)^{2}\right. \\
\times & \left.\left.\left.\times \beta_{0}^{2} \ln ^{2}\left(\frac{\boldsymbol{k}^{2}}{\mu^{2}}\right)-\left(2 \beta_{0} a_{1}+\beta_{1}\right) \ln \left(\frac{\boldsymbol{k}^{2}}{\mu^{2}}\right)+a_{2}\right]\right\}, \quad 22\right) \\
\tilde{V}_{\mathrm{BF}}\left(\boldsymbol{k}, \boldsymbol{k}^{\prime}\right)= & \frac{\pi C_{F} a_{s}}{M_{t}^{2}}+\frac{4 \pi C_{F} a_{s}}{M_{t}^{2}}\left[\boldsymbol{S}_{t} \boldsymbol{S}_{t}^{-}-\frac{\left(\boldsymbol{Q S} \boldsymbol{S}_{t}\right)\left(\boldsymbol{Q S} \boldsymbol{S}_{t}^{-}\right)}{\boldsymbol{Q}^{2}}\right] \\
& -\frac{\pi C_{F} a_{s}}{M_{t}^{2}}\left[\frac{\left(\boldsymbol{k}+\boldsymbol{k}^{\prime}\right)^{2}}{\boldsymbol{Q}^{2}}-\frac{\left(\boldsymbol{k}^{2}-\boldsymbol{k}^{\prime 2}\right)^{2}}{\boldsymbol{Q}^{4}}\right] \\
& +6 i \frac{\pi C_{F} a_{s}}{M_{t}^{2}} \frac{\left(\boldsymbol{S}_{t}+\boldsymbol{S}_{t}^{-}\right)\left(\boldsymbol{k} \times \boldsymbol{k}^{\prime}\right)}{\boldsymbol{Q}^{2}}, \\
\tilde{V}_{\mathrm{NA}}(\boldsymbol{k})= & -\frac{\pi^{2} C_{A} C_{F} a_{s}^{2}}{M_{t} \mid \boldsymbol{k}},
\end{aligned}
$$

where $S_{t}$ and $S_{t}^{-}$are the top and antitop spin operators and $\left(n_{l}=5\right)$

$$
\beta_{0}=\frac{11}{3} C_{A}-\frac{4}{3} T n_{l},
$$

$$
\begin{gathered}
\beta_{1}=\frac{34}{3} C_{A}^{2}-\frac{20}{3} C_{A} T n_{l}-4 C_{F} T n_{l}, \\
a_{1}=\frac{31}{9} C_{A}-\frac{20}{9} \operatorname{Tn}_{l},
\end{gathered}
$$

$$
\begin{aligned}
a_{2}= & \left(\frac{4343}{162}+4 \pi^{2}-\frac{\pi^{4}}{4}+\frac{22}{3} \zeta_{3}\right) C_{A}^{2} \\
& -\left(\frac{1798}{81}+\frac{56}{3} \zeta_{3}\right) C_{A} T n_{l}-\left(\frac{55}{3}-16 \zeta_{3}\right) C_{F} T n_{l} \\
& +\left(\frac{20}{9} T n_{l}\right)^{2} .
\end{aligned}
$$


The constants $\beta_{0}$ and $\beta_{1}$ are the one- and two-loop coefficients of the QCD beta function and $\gamma=0.577216 \cdots$ is the Euler constant; $V_{c}$ is the Coulomb (static) potential. Its $\mathcal{O}\left(\alpha_{s}\right)$ and $\mathcal{O}\left(\alpha_{s}^{2}\right)$ corrections, which come from loops carrying soft momenta, have been determined in Refs. [26,27] and $[28,29]$, respectively. ${ }^{2} V_{\mathrm{BF}}$ is the Breit-Fermi potential known from positronium. It describes the Darwin and the spin-orbit interactions mediated by longitudinal gluons and the hyperfine interactions mediated by transverse gluons in the potential momentum region. Then, $V_{\mathrm{NA}}$ is a purely nonAbelian potential generated (in Coulomb gauge) through a nonanalytic term in a soft momentum one-loop vertex correction to the Coulomb potential involving the triple gluon vertex [30,31] (see also Ref. [32]).

We have already noted that it is a nontrivial fact that ultrasoft gluons do not contribute at NNLO, which means that a common two-body Schrödinger equation, i.e., a wave equation containing an instantaneous interaction potential, is indeed capable of resumming all the terms that we count as NNLO. Although this is a well accepted fact for positronium and the hydrogen atom in QED [33], it is not at all trivial to understand, even in the QED case, if one has to rely on arguments that are not in the framework of (P)NRQCD. In QED the corrections caused by ultrasoft photons are known as retardation effects. The Lamb shift in hydrogen is the most famous example. Based on the identification of the various momentum regions for quarks and gluons mentioned above, however, one has transparent power counting rules at hand; these show that the non-instantaneous exchange of gluons among the top quarks does not lead to any effects at NNLO. For the validity of the argument it is important that the scale $M_{t} \mathrm{v}^{2}$ is much larger than the typical hadronization scale $\Lambda_{\mathrm{QCD}} \cdot{ }^{3}$ To see that retardation effects are suppressed by at least three powers of $v$ with respect to the nonrelativistic limit (LO), we recall that the only source of noninstantaneous interactions in PNRQCD are the ultrasoft gluons, for which all momentum components are of order $M_{t} \mathrm{~V}^{2}$. In addition, only transversely polarized gluons need to be considered as ultrasoft, since we can work in the Coulomb gauge where the time component of the longitudinal gluons vanishes. Thus, an exchange of an ultrasoft gluon among the heavy quark-antiquark pair is already suppressed by $v^{2}$ with respect to LO from the coupling of transverse gluons to the heavy quarks. To see that an additional power of $v$ arises from the loop integration over the ultrasoft gluon momentum, we compare the $v$ counting of the integration measure and the gluon propagator for ultrasoft and potential momenta. In the ultrasoft case, the product of the integration measure $d^{4} k$ and the gluon propagator $1 / k^{2}$ counts as $\mathrm{v}^{8}$ $\times \mathrm{v}^{-4}=\mathrm{v}^{4}$, whereas in the potential case the result reads

\footnotetext{
${ }^{2}$ The constant $a_{2}$ was first calculated in Ref. [29]. In Ref. [28] an error in the coefficient of the term $\propto \pi^{2} C_{A}^{2}$ was corrected.

${ }^{3}$ Even for energies very close to threshold, the scale $M_{t} \mathrm{v}^{2}$ cannot become smaller than $\Gamma_{t}$ since the dominant effect of the top width is to effectively shift the energy into the positive complex plane by an amount $\Gamma_{t}$ (see Sec. V).
}

$v^{5} \times v^{-2}=v^{3}$. Because the potential momenta contribute at LO we find that the ultrasoft gluons can indeed only lead to effects beyond NNLO. Even if the gluon self-coupling is taken into account, this conclusion remains true, because it also leads to additional powers of $\mathrm{v}$ if ultrasoft momenta are involved. We note that in arbitrary gauge the conclusions are true only after all gauge cancellations have been taken into account. The relation $M_{t} \mathrm{v}^{2} \gg \Lambda_{\mathrm{QCD}}$ is needed for the above argumentation: otherwise, the coupling of ultrasoft gluons to the heavy quarks or among themselves, $\alpha_{s}\left(M_{t} \mathrm{v}^{2}\right)$, could be of order 1 . Therefore our conclusion that retardation effects do not contribute at NNLO would not be valid for the bottom quark $^{4}$ or even the charm quark case.

\section{LIPPMANN-SCHWINGER EQUATION}

The nonrelativistic current correlators in the NRQCD factorization formulas for the total top-quark-top-antiquark cross section close to threshold, Eqs. (12) and (13), are directly related to the Green function $\widetilde{G}\left(\boldsymbol{k}, \boldsymbol{k}^{\prime} ; q^{2}\right)$ of the Schrödinger equation (19), which describes off-shell elastic scattering of a top-quark-top-antiquark pair with center-of-mass three momentum $\pm \boldsymbol{k}$ into a top-quark-top-antiquark pair with three momentum $\pm \boldsymbol{k}^{\prime}$. We emphasize that the Green function does not describe the scattering of on-shell top quarks because the common three-dimensional formulation in the form of the Schrödinger equation (19) already contains an implicit integration over the zero components of the momenta in the heavy quark propagators. Because the heavy quark potential is energy independent this integration is trivial by residues. In the first part of this section we give the relations of the current correlators to the Green function in the three-dimensional formulation. In the second part, we present the generalization to four dimensions for those results that are essential for a proper treatment of the $W^{+} W^{-} b \bar{b}$ phase space once the top-quark width is taken into account. For this section we still assume that the top quark is stable.

Taking into account the partial wave decomposition of the Green function of the Schrödinger equation (19)

$$
\tilde{G}\left(\boldsymbol{k}, \boldsymbol{k}^{\prime}\right)=\sum_{l=0}^{\infty} \widetilde{G}^{l}\left(\boldsymbol{k}, \boldsymbol{k}^{\prime}\right),
$$

where $l$ is the total angular momentum quantum number, we find the following relation between the NRQCD nonrelativistic current correlators (14) and (15) and the $S$ and $P$ wave contributions to $\widetilde{G}$ :

$$
\begin{aligned}
\mathcal{A}^{\mathrm{v}}\left(q^{2}\right)= & N_{c} \operatorname{Tr} \int \frac{d^{3} \boldsymbol{k}}{(2 \pi)^{3}} \int \frac{d^{3} \boldsymbol{k}^{\prime}}{(2 \pi)^{3}} \boldsymbol{\sigma}\left(1+\frac{\boldsymbol{k}^{2}}{6 M_{t}^{2}}\right) \\
& \times \widetilde{G}^{0}\left(\boldsymbol{k}, \boldsymbol{k}^{\prime}\right)\left(1+\frac{\boldsymbol{k}^{\prime 2}}{6 M_{t}^{2}}\right) \boldsymbol{\sigma}
\end{aligned}
$$

\footnotetext{
${ }^{4}$ For the case of bottom-quark-bottom-antiquark quark sum rules the conclusions can, however, still be correct if the effective smearing range is chosen larger than $\Lambda_{\mathrm{QCD}}$ [34].
} 


$$
\begin{aligned}
= & 6 N_{c} \int \frac{d^{3} \boldsymbol{k}}{(2 \pi)^{3}} \int \frac{d^{3} \boldsymbol{k}^{\prime}}{(2 \pi)^{3}}\left(1+\frac{\boldsymbol{k}^{2}}{6 M_{t}^{2}}\right) \\
& \times \widetilde{G}^{0}\left(\boldsymbol{k}, \boldsymbol{k}^{\prime}\right)\left(1+\frac{\boldsymbol{k}^{\prime 2}}{6 M_{t}^{2}}\right), \\
\mathcal{A}^{a}\left(q^{2}\right)= & N_{c} \operatorname{Tr} \int \frac{d^{3} \boldsymbol{k}}{(2 \pi)^{3}} \int \frac{d^{3} \boldsymbol{k}^{\prime}}{(2 \pi)^{3}} \frac{\boldsymbol{k} \times \boldsymbol{\sigma}}{M_{t}} \tilde{G}^{1}\left(\boldsymbol{k}, \boldsymbol{k}^{\prime}\right) \frac{\boldsymbol{k}^{\prime} \times \boldsymbol{\sigma}}{M_{t}} \\
= & 4 N_{c} \int \frac{d^{3} \boldsymbol{k}}{(2 \pi)^{3}} \int \frac{d^{3} \boldsymbol{k}^{\prime}}{(2 \pi)^{3}} \frac{\boldsymbol{k} \boldsymbol{k}^{\prime}}{M_{t}^{2}} \tilde{G}^{1}\left(\boldsymbol{k}, \boldsymbol{k}^{\prime}\right),
\end{aligned}
$$

where a proper UV regularization is understood. For simplicity we have dropped the energy argument of the Green function $\widetilde{G}$. In this work we use the Lippmann-Schwinger equation, the Fourier transform of Eq. (19) with respect to $\boldsymbol{k}^{\prime}$,

$$
\begin{aligned}
& {\left[\frac{\boldsymbol{k}^{2}}{M_{t}}-\frac{\boldsymbol{k}^{4}}{4 M_{t}^{3}}-\left(\frac{p_{0}^{2}}{M_{T}}-\frac{p_{0}^{4}}{4 M_{t}^{3}}\right)\right] G(\boldsymbol{k}, \boldsymbol{x})} \\
& \quad=\exp (i \boldsymbol{k} \boldsymbol{x})-\int \frac{d^{3} \boldsymbol{p}^{\prime}}{(2 \pi)^{3}} \tilde{V}\left(\boldsymbol{k}, \boldsymbol{p}^{\prime}\right) G\left(\boldsymbol{p}^{\prime}, \boldsymbol{x}\right),
\end{aligned}
$$

to derive integral equations for $\widetilde{G}^{0}$ and $\widetilde{G}^{1}$, which are then solved numerically. Using the partial wave decomposition of $\exp (i \boldsymbol{k} \boldsymbol{x})(k \equiv|\boldsymbol{k}|, x \equiv|\boldsymbol{x}|)$ :

$$
\exp (i \boldsymbol{k} \boldsymbol{x})=\exp (i k x \cos \theta)=\sum_{l=0}^{\infty} i^{l}(2 l+1) j_{l}(k x) P_{l}(\cos \theta),
$$

where $j_{l}$ are the spherical Bessel functions

$$
j_{l}(x)=(-x)^{l}\left(\frac{1}{x} \frac{d}{d x}\right)^{l}\left[\frac{\sin x}{x}\right]
$$

and $P_{l}$ the Legendre polynomials, one arrives at the following equations for $G^{0}$ and $G^{1}$ :

$$
\begin{gathered}
{\left[\frac{k^{2}}{M_{t}}-\frac{k^{4}}{4 M_{t}^{3}}-\left(\frac{p_{0}^{2}}{M_{t}}-\frac{p_{0}^{4}}{4 M_{t}^{3}}\right)\right] G^{0}(\boldsymbol{k}, \boldsymbol{x})} \\
=\frac{\sin (k x)}{k x}-\int \frac{d^{3} \boldsymbol{p}^{\prime}}{(2 \pi)^{3}} \tilde{V}\left(\boldsymbol{k}, \boldsymbol{p}^{\prime}\right) G^{0}\left(\boldsymbol{p}^{\prime}, \boldsymbol{x}\right), \\
{\left[\frac{\boldsymbol{k}^{2}}{M_{t}}-\frac{p_{0}^{2}}{M_{t}}\right] G^{1}(\boldsymbol{k}, \boldsymbol{x})} \\
=3 i \frac{\boldsymbol{k} \boldsymbol{x}}{k^{2} x^{2}}\left(\frac{\sin (k x)}{k x}-\cos (k x)\right) \\
\quad-\int \frac{d^{3} \boldsymbol{p}^{\prime}}{(2 \pi)^{3}} \tilde{V}\left(\boldsymbol{k}, \boldsymbol{p}^{\prime}\right) G^{1}\left(\boldsymbol{p}^{\prime}, \boldsymbol{x}\right) .
\end{gathered}
$$

Because in Eq. (30) only $S$-wave states are considered, one can, instead of the complicated form of $\widetilde{V}_{\mathrm{BF}}$, use the angular average with respect to the angle between $\boldsymbol{p}^{\prime}$ and $\boldsymbol{k}$ of the
$1 / Q^{4}$ term on the RHS of Eq. (23). Evaluating also the spin matrices for the $S$-wave state, the Breit-Fermi potential simplifies to

$$
\begin{aligned}
\tilde{V}_{\mathrm{BF}}^{s}\left(\boldsymbol{k}, \boldsymbol{k}^{\prime}\right) & =\frac{1}{4 \pi} \int d \Omega \widetilde{V}_{\mathrm{BF}}\left(\boldsymbol{k}, \boldsymbol{k}^{\prime}\right) \\
& =\frac{11}{3} \frac{\pi C_{F} a_{s}}{M_{t}^{2}}-2 \frac{\pi C_{F} a_{s}}{M_{t}^{2}} \frac{\boldsymbol{k}^{2}+\boldsymbol{k}^{\prime 2}}{\boldsymbol{Q}^{2}} .
\end{aligned}
$$

In Eq. (31), on the other hand, we will just use the LO Coulomb potential because the axial-vector contribution to the total cross section is already suppressed by $v^{2}$. For the same reason we do not include any kinematic relativistic corrections in Eq. (31). Defining the $S$-wave and the $P$-wave vertex Green function as

$$
S(\boldsymbol{k})=\int \frac{d^{3} \boldsymbol{p}^{\prime}}{(2 \pi)^{3}} \widetilde{G}^{0}\left(\boldsymbol{k}, \boldsymbol{p}^{\prime}\right)\left(1+\frac{\mathbf{p}^{\prime 2}}{6 M_{t}^{2}}\right),
$$

$$
P(\boldsymbol{k})=\int \frac{d^{3} \mathbf{p}^{\prime}}{(2 \pi)^{3}} \frac{\boldsymbol{k} \boldsymbol{p}^{\prime}}{\boldsymbol{k}^{2}} \tilde{G}^{1}\left(\boldsymbol{k}, \boldsymbol{p}^{\prime}\right),
$$

we finally arrive at the integral equations for $S(\boldsymbol{k})$ and $P(\boldsymbol{k})$,

$$
S(\boldsymbol{k})=G^{f}(\boldsymbol{k})\left(1+\frac{\boldsymbol{k}^{2}}{6 M_{t}^{2}}\right)-G^{f}(\boldsymbol{k}) \int \frac{d^{3} \boldsymbol{p}^{\prime}}{(2 \pi)^{3}} \tilde{V}\left(\boldsymbol{k}, \boldsymbol{p}^{\prime}\right) S\left(\boldsymbol{p}^{\prime}\right),
$$

$$
P(\boldsymbol{k})=G^{f}(\boldsymbol{k})-G^{f}(\boldsymbol{k}) \int \frac{d^{3} \boldsymbol{p}^{\prime}}{(2 \pi)^{3}} \frac{\boldsymbol{k} \boldsymbol{p}^{\prime}}{\boldsymbol{k}^{3}} \widetilde{V}_{c}^{\mathrm{LO}}\left(\boldsymbol{k}, \boldsymbol{p}^{\prime}\right) P\left(\boldsymbol{p}^{\prime}\right),
$$

where

$$
G^{f}(\boldsymbol{k})=\frac{M_{t}}{\boldsymbol{k}^{2}-p_{0}^{2}-i \epsilon}\left[1+\frac{\boldsymbol{k}^{2}+p_{0}^{2}}{4 M_{t}^{2}}\right]
$$

is the free vertex function. We note that $(2 \pi)^{3} \delta^{(3)}(\boldsymbol{k}$ $\left.-\boldsymbol{k}^{\prime}\right) G^{f}(\boldsymbol{k})$ is the Green function of Eq. (19) for $\widetilde{V}=0$.

The vertex functions $S(\boldsymbol{k})$ and $P(\boldsymbol{k})$ only depend on the spatial momentum $\boldsymbol{k}$. As mentioned at the beginning of this section, their dependence on the time component $k^{0}$ has been eliminated by a trivial integration by residues. For a proper integration over the phase space of the top-quark-topantiquark decay products, which is carried out in Sec. V, we also need the full dependence of the vertex functions on $k^{0}$. It can be recovered by comparing $G^{f}(\boldsymbol{k})$ to the product of a free PNRQCD top-quark and top-antiquark propagator at 
NNLO carrying momenta $k_{t}=\left[k^{0}+\left(p_{0}^{2} / 2 M_{t}-p_{0}^{4} / 8 M_{t}^{3}\right), \boldsymbol{k}\right]$ and $k_{t}^{-}=\left[k^{0}-\left(p_{0}^{2} / 2 M_{t}-p_{0}^{4} / 8 M_{t}^{3}\right), \boldsymbol{k}\right]$, respectively: ${ }^{5}$

$$
\begin{aligned}
G^{f}\left(k^{0}, \boldsymbol{k}\right) & \equiv \frac{i}{k^{0}+\left(p_{0}^{2} / 2 M_{t}-p_{0}^{4} / 8 M_{t}^{3}\right)-\left(\boldsymbol{k}^{2} / 2 M_{t}-\boldsymbol{k}^{4} / 8 M_{t}^{3}\right)+i \epsilon} \frac{i}{k^{0}-\left(p_{0}^{2} / 2 M_{t}-p_{0}^{4} / 8 M_{t}^{3}\right)+\left(\boldsymbol{k}^{2} / 2 M_{t}-\boldsymbol{k}^{4} / 8 M_{t}^{3}\right)-i \boldsymbol{\epsilon}} \\
& =\frac{-1}{k^{0^{2}}-\left(p_{0}^{2} / 2 M_{t}-\boldsymbol{k}^{2} / 2 M_{t}+i \epsilon\right)^{2}}+\frac{\left(p_{0}^{2} / 2 M_{t}-\boldsymbol{k}^{2} / 2 M_{t}\right)^{2}\left(p_{0}^{2} / 2 M_{t}^{2}+\boldsymbol{k}^{2} / 2 M_{t}^{2}\right)}{\left[k^{0^{2}}-\left(p_{0}^{2} / 2 M_{t}-\boldsymbol{k}^{2} / 2 M_{t}+i \epsilon\right)^{2}\right]^{2}} .
\end{aligned}
$$

We emphasize that an expansion of the NNLO relativistic effects is understood. The relation between $G^{f}(\boldsymbol{k})$ and $G^{f}\left(k^{0}, \boldsymbol{k}\right)$ reads

$$
G^{f}(\boldsymbol{k})=-i \int_{-\infty}^{+\infty} \frac{d k^{0}}{2 \pi} G^{f}\left(k^{0}, \boldsymbol{k}\right)
$$

Recalling that the potentials as well as the production and annihilation vertex corrections do not depend on the zero components of the momenta, the integral equations for the generalized vertex functions read

$$
\begin{aligned}
& S\left(k^{0}, \boldsymbol{k}\right)=G^{f}\left(k^{0}, \boldsymbol{k}\right)\left(1+\frac{\boldsymbol{k}^{2}}{6 M_{t}^{2}}\right)+i G^{f}\left(k^{0}, \boldsymbol{k}\right) \int \frac{d^{3} \boldsymbol{p}^{\prime}}{(2 \pi)^{3}} \\
& \times \int_{-\infty}^{+\infty} \frac{d p^{\prime 0}}{2 \pi} \tilde{V}\left(\boldsymbol{k}, \boldsymbol{p}^{\prime}\right) S\left(p^{\prime 0}, \boldsymbol{p}^{\prime}\right), \\
& P\left(k^{0}, \boldsymbol{k}\right)=G^{f}\left(k^{0}, \boldsymbol{k}\right)+i G^{f}\left(k^{0}, \boldsymbol{k}\right) \int \frac{d^{3} \boldsymbol{p}^{\prime}}{(2 \pi)^{3}} \\
& \times \int_{-\infty}^{+\infty} \frac{d p^{\prime 0}}{2 \pi} \frac{\boldsymbol{k} \boldsymbol{p}^{\prime}}{k^{2}} \tilde{V}_{c}^{\mathrm{LO}}\left(\boldsymbol{k}, \boldsymbol{p}^{\prime}\right) P\left(p^{\prime 0}, \boldsymbol{p}^{\prime}\right) .
\end{aligned}
$$

The three- and four-dimensional versions of the vertex functions $S$ and $P$ are related by an equation similar to Eq. (39). We note that, by construction, the relation

$$
\frac{X\left(k^{0}, \boldsymbol{k}\right)}{G^{f}\left(k^{0}, \boldsymbol{k}\right)}=\frac{X(\boldsymbol{k})}{G^{f}(\boldsymbol{k})}, \quad(X=S, P)
$$

holds for the amputated vertex functions.

It is straightforward to formulate the optical theorem, which relates the imaginary part of the correlators $\mathcal{A}^{\mathrm{v}}$ and $\mathcal{A}^{a}$ to explicit phase space integrals over the modulus squared of the vertex functions $S$ and $P$. The relations read

\footnotetext{
${ }^{5}$ This choice corresponds to a situation in top-quark-topantiquark ladder diagrams where half of the center-of-mass energy is flowing through the top and half through the antitop line, see Fig. 1.
}

$$
\begin{aligned}
\operatorname{Im}\left[\mathcal{A}^{\mathrm{v}}\left(q^{2}\right)\right]= & 12 \pi^{2} N_{c} \int \frac{d^{3} \boldsymbol{k}}{(2 \pi)^{3}} \int_{-\infty}^{+\infty} \frac{d k^{0}}{2 \pi}\left|\frac{S\left(k^{0}, \boldsymbol{k}\right)}{G^{f}\left(k^{0}, \boldsymbol{k}\right)}\right|^{2} \\
& \times \delta\left[\frac{\boldsymbol{k}^{2}}{2 M_{t}}-\frac{\boldsymbol{k}^{4}}{8 M_{t}^{3}}-\left(\frac{p_{0}^{2}}{2 M_{t}}-\frac{p_{0}^{4}}{8 M_{t}^{3}}\right)+k^{0}\right] \\
& \times \delta\left[\frac{\boldsymbol{k}^{2}}{2 M_{t}}-\frac{\boldsymbol{k}^{4}}{8 M_{t}^{3}}-\left(\frac{p_{0}^{2}}{2 M_{t}}-\frac{p_{0}^{4}}{8 M_{t}^{3}}\right)-k^{0}\right] \\
= & 6 \pi N_{c} \int \frac{d^{3} \boldsymbol{k}}{(2 \pi)^{3}}\left|\frac{S(\boldsymbol{k})}{G^{f}(\boldsymbol{k})}\right|^{2} \\
& \times \delta\left[\frac{\boldsymbol{k}^{2}}{M_{t}}-\frac{\boldsymbol{k}^{4}}{4 M_{t}^{3}}-\left(\frac{p_{0}^{2}}{M_{t}}-\frac{p_{0}^{4}}{4 M_{t}^{3}}\right)\right], \\
\operatorname{Im}\left[\mathcal{A}^{a}\left(q^{2}\right)\right]= & 8 \pi^{2} N_{c} \int \frac{d^{3} \boldsymbol{k}}{(2 \pi)^{3}} \int_{-\infty}^{+\infty} \frac{d k^{0}}{2 \pi} \frac{\boldsymbol{k}^{2}}{M_{t}^{2}}\left|\frac{P\left(k^{0}, \boldsymbol{k}\right)}{G^{f}\left(k^{0}, \boldsymbol{k}\right)}\right|^{2} \\
& \times \delta\left(\frac{\boldsymbol{k}^{2}}{2 M_{t}}-\frac{p_{0}^{2}}{2 M_{t}}+k^{0}\right) \delta\left(\frac{\boldsymbol{k}^{2}}{2 M_{t}}-\frac{p_{0}^{2}}{2 M_{t}}-k^{0}\right) \\
= & 4 \pi N_{c} \int \frac{d^{3} \boldsymbol{k}}{(2 \pi)^{3}} \frac{\boldsymbol{k}^{2}}{M_{t}^{2}}\left|\frac{P(\boldsymbol{k})}{G^{f}(\boldsymbol{k})}\right|^{2} \delta\left(\frac{\boldsymbol{k}^{2}}{M_{t}}-\frac{p_{0}^{2}}{M_{t}}\right) .
\end{aligned}
$$

\section{REGULARIZATION SCHEME AND SHORT-DISTANCE COEFFICIENTS}

All equations derived previously have to be considered in the framework of a proper UV regularization scheme. In fact, UV linear and logarithmic divergences arise in Eq. (35) from the NNLO non-Coulombic potentials and from the kinetic energy and vertex corrections. However, we emphasize that even in the case when no UV divergences arise, all integrals have to be consistently regularized, because only in a consistent regularization scheme can the short-distance coefficients be defined properly. In principle, the regularization scheme "of choice" would be an analytic scheme such as $\overline{\mathrm{MS}}$ as it is usually used in modern perturbative QCD calculations. The preference for the $\overline{\mathrm{MS}}$ scheme arises from the fact that it naturally preserves gauge invariance, Ward identities and, particularly important in the framework of effec- 


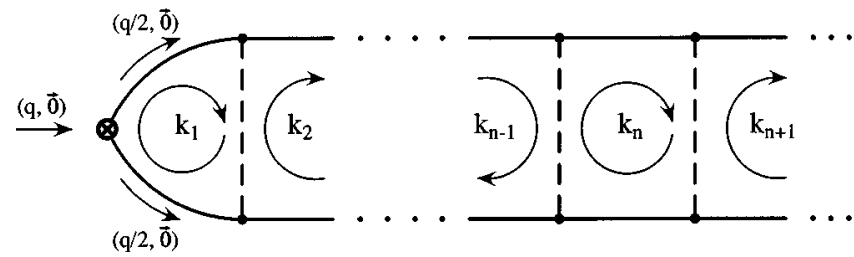

FIG. 1. Routing convention for loop momenta in ladder diagrams.

tive field theories, power counting rules. Unfortunately an analytic solution of Eqs. (19), (35), and (36) is not even known for four dimensions. Thus, the only sensible way to use the $\overline{\mathrm{MS}}$ scheme is to start from the known Coulomb solution of the nonrelativistic Schrödinger equation and include NLO and NNLO corrections via time-independent perturbation theory, and then explicitly construct the spectral representation of the Green function at the NNLO level. While this program might still be feasible for the determination of the total cross section, it is rather cumbersome for the calculations of distributions at NNLO.

In this work we use a momentum cutoff regularization scheme by simply excluding momenta that have a spatial component larger than the cutoff $\Lambda$. This is in fact the most natural regularization scheme for a numerical solution of Eqs. (35) and (36). However, a cutoff scheme contains a number of subtleties, which shall be briefly discussed in the following. As indicated before, a cutoff scheme leads to violations of gauge invariance and Ward identities in the (P)NRQCD calculation. These effects, however, are generated at the cutoff and are therefore cancelled by corresponding terms with a different sign in the short-distance coefficients. Thus the cross section, which contains the proper combination of nonrelativistic correlators and short-distance coefficients, is gauge-invariant and satisfies all Ward identities up to terms beyond the order at which the matching calculation has been carried out. Another subtlety of a cutoff prescription is that it is only well defined if a specific routing convention for the momenta in loops is adopted. For the calculations of the top-antitop cross section at NNLO, it is straightforward and easy to find such a routing convention, because only ladder-type diagrams are involved. It is natural to choose the routing used in the integral equations (35) and (36), which we have, for clarity, depicted graphically in Fig. 1. It is important to use exactly the same routing for the (P)NRQCD diagrams calculated in the matching procedure to obtain consistent results for the NNLO short-distance coefficients. Finally, it has to be mentioned that a cutoff scheme inevitably leads to power counting breaking effects. This means in our case that a term in the Schrödinger equation (19), which is NNLO according to the velocity counting, can in principle lead to lower order contributions in the nonrelativistic current correlator. Similar to the terms that violate gauge invariance and Ward identities, the power counting breaking terms are also generated at the cutoff; they are therefore cancelled in the combination of the correlators and the corresponding short-distance coefficients. However, all this happens only if the cutoff is chosen of the order of $M_{t}$.
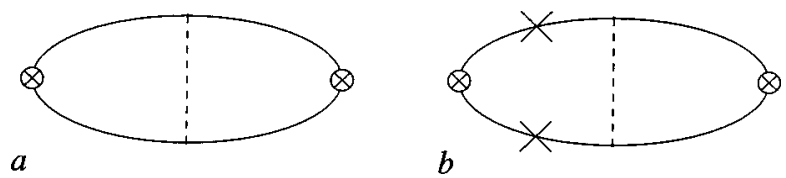

FIG. 2. The two-loop NRQCD vector current correlator with the exchange of one Coulomb gluon without relativistic corrections (a) and with the kinetic energy corrections (b).

To illustrate this issue let us first consider the LO two-loop NRQCD vector current correlator, which contains the exchange of a Coulomb gluon as shown in Fig. 2(a). It is straightforward to calculate the absorptive part of the diagram as an expansion in $p_{0}$ (see the Appendix):

$$
I_{1}^{(1)}=\frac{C_{F} \alpha_{s} M_{t}^{2}}{4 \pi^{2}}\left[\frac{\pi^{2}}{2}-\frac{4 p_{0}}{\Lambda}+\mathcal{O}\left(p_{0}^{3}\right)\right] .
$$

The first term in the brackets on the RHS of Eq. (45) is the well known Coulomb singularity, which leads to a finite cross section at order $\alpha_{s}$. The second term is cutoffdependent and leads to a short-distance correction $\propto\left(\alpha_{s} / \pi\right)\left(M_{t} / \Lambda\right)$. (In the Appendix the reader can convince her/himself that the scale of $\alpha_{s}$ in this term is indeed of order $M_{t}$.) Obviously, to avoid a breakdown in the separation of long- and short-distance contributions, ${ }^{6} \Lambda$ has to be chosen of order $M_{t}$. (Choosing $\Lambda$ of order $M_{t} \vee$ would be absurd anyway, because this would cut off a large part of the dynamics. In this respect the cutoff $\Lambda$ acts in a way completely different from the "cutoff" scale in the $\overline{\mathrm{MS}}$ scheme, which would naturally be chosen of order $M_{t} \mathrm{v}$.) Let us now consider the kinetic energy corrections in the same two-loop NRQCD diagram, as shown in Fig. 2(b). Using the result obtained in the Appendix the expression for the diagram including combinatorial factors reads

$$
I_{3}^{(1)}=\frac{C_{F} \alpha_{s} M_{t}^{2}}{4 \pi^{2}}\left[\frac{\Lambda p_{0}}{M_{t}^{2}}+\frac{p_{0}^{2} \pi^{2}}{2 M_{t}^{2}}+\mathcal{O}\left(p_{0}^{3}\right)\right] .
$$

As expected from power counting arguments, the kinetic energy correction leads to a contribution suppressed by $p_{0}^{2} / M_{t}^{2}$ with respect to the pure Coulomb exchange diagram in Eq. (45). However, there is also a term proportional to $p_{0} / M_{t}$ because $\Lambda$ is of order $M_{t}$. This is an example for a power counting breaking term. In the short-distance coefficient $C^{\mathrm{v}}$ this term leads to a NNLO contribution $\propto \alpha_{s}\left(\Lambda / M_{t}\right)$. Because this term only arises if NNLO relativistic effects are taken into account, we have to count it as NNLO. Similar terms are caused by the Breit-Fermi potential $V_{\mathrm{BF}}$, the nonAbelian potential $V_{\mathrm{NA}}$ and the dimension-5 NRQCD vector current. We emphasize again that, as is the case for the terms that violate gauge invariance and Ward identities, all power counting breaking terms are automatically cancelled in the

\footnotetext{
"In this context "long-distance", effects are not understood as "nonperturbative", effects, which come from scales of order $\Lambda_{\mathrm{QCD}}$, but rather as effects governed by scales of order $M_{t} \mathrm{v}$ or $M_{t} \mathrm{v}^{2}$.
} 
combination with the short-distance coefficients up to terms beyond the order at which one carries out the matching procedure. In our case, where the matching is carried out at order $\alpha_{s}^{2}$, power counting breaking terms of order $\alpha_{s}^{3}$ remain uncancelled, but they are beyond NNLO accuracy.

Taking into account the issues discussed above, it is straightforward to determine the matching coefficient $C^{\mathrm{v}}$. Details of this calculation are given in the Appendix. The result reads $\left[a_{s} \equiv \alpha_{s}(\mu)\right]$

$$
\begin{aligned}
C^{\vee}= & +\left\{\frac{4 C_{F} a_{s}}{\pi}\left[-1+\frac{M_{t}}{\Lambda}\right]\right\}^{\mathrm{NLO}}+\left(\frac{4 C_{F} a_{s} \Lambda}{3 \pi M_{t}}\right. \\
& +\frac{C_{F}^{2} a_{s}^{2}}{\pi^{2}}\left\{\frac { \beta _ { 0 } } { C _ { F } } \left[-\frac{\Lambda^{2}+12 M_{t}^{2}}{6 \Lambda M_{t}}+\frac{\Lambda^{2}-12 M_{t}^{2}}{6 \Lambda M_{t}} \ln \left(\frac{\Lambda}{\mu}\right)\right.\right. \\
& \left.+2 \ln \left(\frac{M_{t}}{\mu}\right)\right]-\frac{a_{1}}{C_{F}} \frac{\Lambda^{2}-12 M_{t}^{2}}{12 \Lambda M_{t}}+\pi^{2}\left(\frac{2}{3}+\frac{C_{A}}{C_{F}}\right) \ln \left(\frac{2 M_{t}}{\Lambda}\right) \\
& +\frac{\pi^{2} \kappa}{C_{F}^{2}}+\frac{16 \Lambda^{2}}{9 M_{t}^{2}}-\frac{16 \Lambda}{3 M_{t}}-\frac{16 M_{t}}{\Lambda}+\frac{M_{t}^{2}\left(20+\pi^{2}\right)}{2 \Lambda^{2}}-\frac{53 \pi^{2}}{24} \\
& \left.\left.-\frac{C_{A} \pi^{2}}{C_{F}}+\frac{25}{6}+\frac{7}{3} \zeta_{3}\right\}\right)
\end{aligned}
$$

As explained in the Appendix we have displayed the NLO and NNLO contributions to $C^{\mathrm{V}}$ separately. The Appendix also contains a discussion on the convergence of the perturbative series in $C^{\mathrm{v}}$ in our cutoff scheme compared to the $\overline{\mathrm{MS}}$ scheme [35,36].

\section{TOP-QUARK WIDTH}

Up to now we have treated the top quark as a stable particle. For top-quark production close to threshold this is not appropriate because the kinetic energy of the top quark $\sim M_{t} \mathrm{v}^{2} \sim M_{t} \alpha_{s}^{2} \sim 2-3 \mathrm{GeV}$ is of the same order as its decay width. It has been shown by Fadin and Khoze [2] that in the nonrelativistic limit the top-quark width can be consistently implemented by calculating the cross section for stable top quarks supplemented by the replacement $E \rightarrow E+i \Gamma_{t}$, where $E$ is the center-of-mass energy measured with respect to the two-particle threshold. For the total cross section, this prescription remains valid even at NLO because, in this case, order $\alpha_{s}$ QCD radiative corrections in form of a gluon that connects top quark production and decay vanish [20]. A consistent implementation of the top-quark width at NNLO has been missing so far.

In the framework of NRQCD, and if one is not interested in any differential information on the top-quark decay products, the top-quark width can be understood as a modification of the NRQCD matching conditions caused by electroweak corrections. Because the particles involved in these corrections can be lighter than the top quark (i.e., if the top quark can decay weakly) they can lead to nonzero imaginary parts in the matching conditions and, likewise, in the shortdistance coefficients of the NRQCD Lagrangian and the NRQCD currents. This is a well known concept in quantum mechanics of inelastic processes where particle decay and absorption processes are represented by potentials and couplings carrying complex coefficients, if one is not interested in the details of the decay and absorption process. In this context the effects of the top-quark decay are only a small (but nevertheless the most important) part of a whole array of electroweak corrections relevant to top-quark-top-antiquark pair production close to threshold. In this work we only consider the effects from the on-shell top-quark decay into a $W$ boson and a bottom quark, assuming that the $W$ and the $b$ are themselves stable. Thus the results presented here are, by definition, gauge invariant. The consistent treatment of all electroweak effects, including a proper handling of the offshell decays of the top quark, interconnection effects and of gauge-invariance-violating contributions, is beyond the scope of this paper. Such a treatment is carried out in Ref. [37].

Before we determine the modifications of the NRQCD Lagrangian, Eq. (1), through the top-quark decay, some remarks about the velocity counting of the top quark width are in order. Comparing the numerical size of the top-quark width

$$
\Gamma_{t} \approx \frac{G_{F}}{\sqrt{2}} \frac{M_{t}^{3}}{8 \pi} \sim 1.5 \mathrm{GeV}
$$

with the binding energy of a fictitious toponium $1 S$ bound state in the pole mass scheme $\left(\alpha_{s} \sim 0.15\right)$

$$
E_{1 S} \approx \frac{M_{t}\left(C_{F} \alpha_{s}\right)^{2}}{4} \sim 1.75 \mathrm{GeV},
$$

we find that we have to count $\Gamma_{t} / M_{t}$ as order $\mathrm{v}^{2}$, i.e.,

$$
\frac{\Gamma_{t}}{M_{t}} \sim \alpha_{s}^{2} \sim \mathrm{v}^{2}
$$

Recalling the velocity counting of the operators of the NRQCD Lagrangian, this means that at NNLO and to first order in $\Gamma_{t} / M_{t}$ we have to determine the coefficients of the NRQCD operators $\psi^{\dagger} i \Gamma_{t} \psi, \quad \psi^{\dagger} i\left(\Gamma_{t} / M_{t}\right) D_{t} \psi$, $\psi^{\dagger} i \Gamma_{t}\left(\boldsymbol{D}^{2} / M_{t}^{2}\right) \psi$ and those operators, where the top-quark Pauli spinors are replaced by the top-antiquark ones. As we show later in this section there are also contributions proportional to $i \Gamma_{t}$ to the photon and $Z$ boson wave function renormalization constants, which have to be included to account for a proper treatment of the top-quark decay phase space. We note that the velocity counting (50) implies that $g \sim \alpha_{s}$ $\sim \mathrm{V}$, where $g$ is the $\mathrm{SU}(2)$ gauge coupling. Thus in a complete calculation of all electroweak effects one also has to determine $\mathcal{O}\left(g^{4}\right)$ electroweak contributions and $\mathcal{O}\left(\alpha_{s}^{2}\right)$ QCD corrections to the top decay width. In the framework of the standard model those corrections are known [38-40]. For our purposes, however, it is sufficient to consider the top- 
quark width as an independent parameter, which we treat only to first order and which is not subject to higher-order corrections.

The matching coefficients of the bilinear top-quark spinor operators given above can be obtained by sandwiching the absorptive part of the top-quark self-energy $\Sigma_{t}$ (in the full electroweak theory) between top-quark Dirac spinors and expanding the result around the complex pole position. Keeping only the terms proportional to $\Gamma_{t}$, the result for the bilinear top-quark Pauli spinor terms reads $\left[k=\left(k^{0}, \boldsymbol{k}\right)\right]$

$$
\begin{aligned}
\bar{u}(\boldsymbol{k})\left[\operatorname{Im} \Sigma_{t}(k)\right] u(\boldsymbol{k}) & \Rightarrow \bar{u}(\boldsymbol{k}) i \frac{\Gamma_{t}}{2} u(\boldsymbol{k}) \\
& =\tilde{\psi}^{\dagger}\left[i \frac{\Gamma_{t}}{2} \frac{M_{t}}{E_{k}}\right] \tilde{\psi} \\
& =\tilde{\psi}^{\dagger}\left[i \frac{\Gamma_{t}}{2}\left(1-\frac{\boldsymbol{k}^{2}}{2 M_{t}^{2}}\right)\right] \tilde{\psi} .
\end{aligned}
$$

The corresponding result for the bilinear top-antiquark Pauli spinor terms reads

$$
\begin{aligned}
\overline{\mathrm{v}}(-\boldsymbol{k})\left[\operatorname{Im} \Sigma_{t}(k)\right] \mathrm{v}(-\boldsymbol{k}) & \Rightarrow \overline{\mathbf{v}}(-\boldsymbol{k}) i \frac{\Gamma_{t}}{2} \mathrm{v}(-\boldsymbol{k}) \\
& =-\tilde{\chi}^{\dagger}\left[i \frac{\Gamma_{t}}{2} \frac{M_{t}}{E_{k}}\right] \tilde{\chi} \\
& =-\tilde{\chi}^{\dagger}\left[i \frac{\Gamma_{t}}{2}\left(1-\frac{\boldsymbol{k}^{2}}{2 M_{t}^{2}}\right)\right] \tilde{\chi},
\end{aligned}
$$

where $E_{k} \equiv\left(\boldsymbol{k}^{2}+M_{t}^{2}\right)^{1 / 2}$ and

$$
\begin{gathered}
u(\boldsymbol{k})=\sqrt{\frac{E_{k}+M_{t}}{2 E_{k}}}\left(\begin{array}{c}
\tilde{\psi} \\
\frac{\boldsymbol{\sigma} \boldsymbol{k}}{E_{k}+M_{t}} \tilde{\psi}
\end{array}\right), \\
\mathbf{v}(-\boldsymbol{k})=\sqrt{\frac{E_{k}+M_{t}}{2 E_{k}}}\left(\begin{array}{c}
-\frac{\boldsymbol{\sigma} \boldsymbol{k}}{E_{k}+M_{t}} \tilde{\chi} \\
\tilde{\chi}
\end{array}\right),
\end{gathered}
$$

using the usual nonrelativistic normalization for Dirac spinors. Thus in the presence of top decay we have to modify the NRQCD Lagrangian by adding the terms

$\delta \mathcal{L}_{\mathrm{NRQCD}}=\psi^{\dagger} i \frac{\Gamma_{t}}{2}\left[1+\frac{D^{2}}{2 M_{t}^{2}}\right] \psi-\chi^{\dagger} i \frac{\Gamma_{t}}{2}\left[1+\frac{\boldsymbol{D}^{2}}{2 M_{t}^{2}}\right] \chi$.

Physically, the dimension-5 operators multiplying the top width correspond to the time dilatation correction. This leads to the following modified versions of the Schrödinger equation (19) in momentum space representation

$$
\begin{gathered}
{\left[\frac{\boldsymbol{k}^{2}}{M_{t}}-\frac{\boldsymbol{k}^{4}}{4 M_{t}^{3}}-\left(\frac{p_{0}^{2}}{M_{t}}-\frac{p_{0}^{4}}{4 M_{t}^{3}}\right)-i \Gamma_{t}\left(1-\frac{\boldsymbol{k}^{2}}{2 M_{t}^{2}}\right)\right] \widetilde{G}\left(\boldsymbol{k}, \boldsymbol{k}^{\prime} ; q^{2}\right)} \\
\quad+\int \frac{d^{3} \boldsymbol{p}^{\prime}}{(2 \pi)^{3}} \tilde{V}\left(\boldsymbol{k}, \boldsymbol{p}^{\prime}\right) \tilde{G}\left(\boldsymbol{p}^{\prime}, \boldsymbol{k}^{\prime} ; q^{2}\right)=(2 \pi)^{3} \delta^{(3)}\left(\boldsymbol{k}-\boldsymbol{k}^{\prime}\right),
\end{gathered}
$$

and the free vertex functions $G^{f}(\boldsymbol{k})$ and $G^{f}\left(k^{0}, \boldsymbol{k}\right)$ in the integral equations (35), (36), (40), and (41):

$$
\begin{aligned}
G^{f}(\boldsymbol{k}) & =\frac{M_{t}}{\boldsymbol{k}^{2}-p_{0}^{2}-\Gamma_{t}^{2} / 4-i M_{t} \Gamma_{t}}\left[1+\frac{\boldsymbol{k}^{2}+p_{0}^{2}}{4 M_{t}^{2}}-i \frac{\Gamma_{t}}{4 M_{t}}\right], \\
G^{f}\left(k^{0}, \boldsymbol{k}\right) & =\frac{-1}{k^{0^{2}}-\left[\left(1 / 2 M_{t}\right)\left(p_{0}^{2}+\Gamma_{t}^{2} / 4\right)-\boldsymbol{k}^{2} / 2 M_{t}+i \Gamma_{t} / 2\right]^{2}}+\frac{\left[p_{0}^{2} / 2 M_{t}-\boldsymbol{k}^{2} / 2 M_{t}+i\left(\Gamma_{t} / 2\right)\right]^{2}\left[p_{0}^{2} / 2 M_{t}^{2}+\boldsymbol{k}^{2} / 2 M_{t}^{2}-i\left(\Gamma_{t} / 2 M_{t}\right)\right]}{\left\{k^{0^{2}}-\left[1 / 2 M_{t}\left(p_{0}^{2}+\Gamma_{t}^{2} / 4\right)-\boldsymbol{k}^{2} / 2 M_{t}+i\left(\Gamma_{t} / 2\right)\right]^{2}\right\}^{2}} .
\end{aligned}
$$

We note that it is not coercive to keep the term $-\Gamma_{t}^{2} / 4$ in the LO propagator, because it is of NNLO according to the power counting. We have adopted this convention because this choice leads to a simplification of the analytic form for the rest of the NNLO corrections in Eqs. (56) and (57). From the modified version of the Schrödinger equation (55) we can immediately see that the Fadin-Khoze replacement rule is valid at LO and NLO in the nonrelativistic expansion, but it is inappropriate at NNLO. In fact it is not possible at all to consistently implement the top-quark width at NNLO simply by shifting the center-of-mass energy in a calculation for stable quarks. The resulting form of the optical theorem relations reads

$$
\begin{gathered}
\operatorname{Im}\left[\mathcal{A}^{\mathrm{v}}\left(q^{2}\right)\right]=3 N_{c} \int \frac{d^{3} \boldsymbol{k}}{(2 \pi)^{3}} \int_{-\infty}^{+\infty} \frac{d k^{0}}{2 \pi}\left|S\left(k^{0}, \boldsymbol{k}\right)\right|^{2} \Gamma_{t}^{2}\left(1-\frac{\boldsymbol{k}^{2}}{M_{t}^{2}}\right)=6 N_{c} \int \frac{d^{3} \boldsymbol{k}}{(2 \pi)^{3}}|S(\boldsymbol{k})|^{2} \Gamma_{t}\left(1-\frac{\boldsymbol{k}^{2}}{2 M_{t}^{2}}\right), \\
\operatorname{Im}\left[\mathcal{A}^{a}\left(q^{2}\right)\right]=2 N_{c} \int \frac{d^{3} \boldsymbol{k}}{(2 \pi)^{3}} \int_{-\infty}^{+\infty} \frac{d k^{0}}{2 \pi} \frac{\boldsymbol{k}^{2}}{M_{t}^{2}}\left|P\left(k^{0}, \boldsymbol{k}\right)\right|^{2} \Gamma_{t}^{2}=4 N_{c} \int \frac{d^{3} \boldsymbol{k}}{(2 \pi)^{3}} \frac{\boldsymbol{k}^{2}}{M_{t}^{2}}|P(\boldsymbol{k})|^{2} \Gamma_{t} .
\end{gathered}
$$


The first equality in Eqs. (58) and (59) is an explicit integration over the phase space of the top-quark decay products for off-shell top decay keeping only the term proportional to the top-quark and top-antiquark width for the top-quark decay subphase space. We emphasize again that in Eqs. (58) and (59) no physical phase space boundaries for the integration over the top-quark-top-antiquark four momentum are implemented. All integrations are defined in the framework of the regularization scheme.

The reader might ask whether the inclusion of the topquark width into the top-quark propagators leads to nontrivial modifications of the NRQCD short-distance coefficient $C^{\mathrm{v}}{ }^{7}$ Because $\Gamma_{t} / M_{t} \sim \mathrm{v}^{2} \sim \alpha_{s}^{2}$, it is not inconceivable that there could be terms $\propto \Gamma_{t} / M_{t}$ in $C^{\vee}$ at NNLO. To show that this is not the case we recall that $C^{\mathrm{V}}$ is the modulus squared of the short-distance coefficient $c_{1}^{\vee}$ of the nonrelativistic current $\psi^{\dagger} \boldsymbol{\sigma} \chi$ in Eq. (2). As mentioned in the Appendix, the short-distance coefficient $c_{1}^{v}$ is the sum of amputated vertex diagrams in the full theory where the three momenta in the loop integrations are larger than the cutoff $\Lambda \sim M_{t}$ for $\sqrt{s}=2 M_{t}$. Thus, there is no integration over the top-quarktop-antiquark pole located at three momenta $\sim p_{0} \sim M_{t} \mathrm{v}$, and we can conclude that the short-distance coefficient does not contain nonanalytic terms involving the top-quark width. Therefore $c_{1}^{v}$ can be expanded in $i \Gamma_{t}$. For the same reason, the coefficients of an expansion in $i \Gamma_{t}$ do not contain a topquark-top-antiquark cut and are real numbers. Because $C^{\mathrm{v}}$ is the modulus squared of $c_{1}^{\mathrm{v}}$, the first nonvanishing term of an expansion of $C^{\mathrm{v}}$ is proportional to $\left(\Gamma_{t} / M_{t}\right)^{2} \sim \alpha_{s}^{4}$, which is indeed beyond NNLO. However, we emphasize that, in a complete calculation of all electroweak effects, $C^{\mathrm{v}}$ will receive corrections $\propto g^{2}, g^{\prime 2} \sim G_{F} M_{W}^{2}, g^{\prime}$ being the U(1) gauge coupling, which are formally of order $\Gamma_{t} / M_{t}$. These corrections do not come from the width contained in the top-quark and top-antiquark propagators but, for instance, from electroweak corrections to the vertices $\gamma, Z \rightarrow t \bar{t}$ [41]. These corrections exist even in the case when the top quark is treated as a stable particle.

If the top-quark width is included in the NRQCD framework there is only one additional source of a linear dependence on $\Gamma_{t}$ which comes from the phase space integration in Eqs. (58) and (59). In contrast to the case of a stable top quark, where the phase space is restricted to those top-quark four momenta allowed by the center-of-mass energy, the physical boundaries of the phase space integration $\int\left[d^{4} k /(2 \pi)^{4}\right]$ in the case of unstable top quarks are determined by the allowed invariant masses of the top-quark decay products. Assuming that the bottom quark is massless and that bottom quark and $W$ boson are stable, and taking into account our routing convention (see Fig. 1), the boundaries of the physical four-dimensional phase space integrations read $\left(k^{2} \equiv|\boldsymbol{k}|^{2}\right)$

\footnotetext{
${ }^{7}$ One could equally well ask whether there is a need to introduce the operators $\left(\Gamma_{t} / M_{t}\right) \psi^{\dagger} \sigma \chi$ and $\left(\Gamma_{t} / M_{t}\right) \chi^{\dagger} \sigma \psi$ in the nonrelativistic expansion of the currents, which produce and annihilate the top-quark-top-antiquark pair. At this point we prefer the language used in the text.
}

$$
\begin{aligned}
\int_{\text {phase space }} \frac{d^{4} k}{(2 \pi)^{4}}= & \int_{0} \sqrt{q^{2} / 4-M_{W}^{2}} \frac{d^{3} \boldsymbol{k}}{(2 \pi)^{3}} \\
& \times \int_{-\left(\sqrt{q^{2}} / 2-\sqrt{k^{2}+M_{W}^{2}}\right)}^{\left(\sqrt{q^{2}} / 2-\sqrt{k^{2}+M_{W}^{2}}\right)} \frac{d k^{0}}{2 \pi} .
\end{aligned}
$$

It is obvious that the physical limits of integration are not equivalent to the actual limits of integration on the RHS of Eqs. (58) and (59) as defined through our cutoff regularization scheme:

$$
\int_{\text {cutoff scheme }} \frac{d^{4} k}{(2 \pi)^{4}}=\int_{0}^{|\boldsymbol{k}|<\Lambda} \frac{d^{3} \boldsymbol{k}}{(2 \pi)^{3}} \int_{-\infty}^{+\infty} \frac{d k^{0}}{2 \pi}
$$

For the total cross section on the LHS of Eqs. (58) and (59), the difference between using the phase space integrations (61) or (60) can be expanded in $p_{0}^{2}$ and $\Gamma_{t}$, and can be accounted for by introducing additional photon and $\mathrm{Z}$ boson wave function renormalization constants into the NRQCD Lagrangian, which are proportional to $i \Gamma_{t}$. The calculation of these counterterms is straightforward. This leads to the following form of the optical theorem relations for the vector- and axial-vector-current-induced correlators

$$
\begin{aligned}
& \operatorname{Im}\left[\mathcal{A}^{\mathrm{v}}+i \frac{3 N_{c} M_{t} \Gamma_{t}}{2 \pi^{2}}\left(2 \frac{M_{t}}{\Lambda}-(1+\sqrt{3})\right)\right] \\
& \quad=3 N_{c} \int_{\text {phase space }} \frac{d^{4} k}{(2 \pi)^{4}}\left|S\left(k^{0}, \boldsymbol{k}\right)\right|^{2} \Gamma_{t}^{2}\left(1-\frac{\boldsymbol{k}^{2}}{M_{t}^{2}}\right) \\
& \operatorname{Im}\left[\mathcal{A}^{a}+i \frac{N_{c} M_{t} \Gamma_{t}}{\pi^{2}}\left(-2 \frac{\Lambda}{M_{t}}+2(\sqrt{3}-1)\right)\right] \\
& \quad=2 N_{c} \int_{\text {phase space }} \frac{d^{4} k}{(2 \pi)^{4}} \frac{\boldsymbol{k}^{2}}{M_{t}^{2}}\left|P\left(k^{0}, \boldsymbol{k}\right)\right|^{2} \Gamma_{t}^{2},
\end{aligned}
$$

where we have displayed the counterterms for the phase space, each at the Born level, to first order in $\Gamma_{t}$ and first order in the nonrelativistic expansion. For simplicity we have set the bottom quark and the $W$ boson masses to zero. As far as the velocity counting of the width is concerned, we in principle should have included also the $\mathcal{O}\left(\alpha_{s}\right)$ contributions for the counterterm in Eq. (62), because we formally have to count the vector-current-induced total cross section as order v. However, the counterterm contributions are only at the per cent level and not (yet) important phenomenologically, compared with the much larger uncertainties in the normalization of the total cross section from QCD, which are discussed in Sec. VIII B. We also note that the phase space counterterms can be calculated entirely within the nonrelativistic effective theory, and that there is no need to match to the full theory. This is because within the physical phase space boundaries the nonrelativistic expansion of the phase 
space integration should be convergent. ${ }^{8}$ We also note that the calculation of the phase space counterterm for the S-Pwave interference, contributing, e.g., in the top-quark angular distribution, is in complete analogy to the calculation of the counterterms in Eqs. (62) and (63). We emphasize that the nonrelativistic current correlators $\mathcal{A}^{\mathrm{v}}, \mathcal{A}^{a}$ and the vertex functions $S$ and $P$ in relations (62) and (63) are still calculated in our cutoff scheme presented in Sec. IV.

The expressions for the vector-current- and axial-vectorcurrent-induced total cross sections valid at NNLO in the nonrelativistic expansion, and properly including all effects of the top-quark width at the Born level and leading order in the nonrelativistic expansion, read

$$
\begin{aligned}
R_{\mathrm{NNLO}}^{\mathrm{v}, \mathrm{thr}}\left(q^{2}\right)= & \frac{4 \pi}{q^{2}} C^{\mathrm{v}} \operatorname{Im}\left[\mathcal{A}^{\mathrm{v}}\left(q^{2}\right)\right] \\
& +\frac{3 N_{c} \Gamma_{t}}{2 \pi M_{t}}\left[2 \frac{M_{t}}{\Lambda}-(1+\sqrt{3})\right], \\
R_{\mathrm{NNLO}}^{a, \mathrm{thr}}\left(q^{2}\right)= & \frac{4 \pi}{q^{2}} C^{a} \operatorname{Im}\left[\mathcal{A}^{a}\left(q^{2}\right)\right] \\
& +\frac{N_{c} \Gamma_{t}}{\pi M_{t}}\left[-2 \frac{\Lambda}{M_{t}}+2(\sqrt{3}-1)\right] .
\end{aligned}
$$

From Eqs. (62) and (63) we can derive the center-of-mass three-momentum distributions of the top quarks $(k \equiv|\boldsymbol{k}|)$,

$$
\begin{aligned}
\frac{d R_{\mathrm{NNLO}}^{\mathrm{v}, \mathrm{thr}}\left(q^{2}\right)}{d|\boldsymbol{k}|}= & C^{\mathrm{v}} \frac{6 N_{c}}{\pi q^{2}} \Gamma_{t}^{2}\left(1-\frac{\boldsymbol{k}^{2}}{M_{t}^{2}}\right) \boldsymbol{k}^{2} \\
& \times \int_{-\left(\sqrt{\left.q^{2} / 2-\sqrt{k^{2}+M_{W}^{2}}\right)}\right.}^{\left(\sqrt{q^{2}} / 2-\sqrt{k^{2} M_{W}^{2}}\right)} \frac{d k^{0}}{2 \pi}\left|S\left(k^{0}, \boldsymbol{k}\right)\right|^{2},
\end{aligned}
$$

$$
\begin{aligned}
\frac{d R_{\mathrm{NNLO}}^{a, \mathrm{thr}}\left(q^{2}\right)}{d|\boldsymbol{k}|}= & C^{a} \frac{4 N_{c}}{\pi q^{2}} \Gamma_{t}^{2} \frac{\boldsymbol{k}^{4}}{M_{t}^{2}} \\
& \times \int_{-\left(\sqrt{q^{2}} / 2-\sqrt{k^{2}+M_{W}^{2}}\right)}^{\left(\sqrt{q^{2}} / 2-\sqrt{k^{2}+M_{2}^{2}}\right)} \frac{d k^{0}}{2 \pi}\left|P\left(k^{0}, \boldsymbol{k}\right)\right|^{2} .
\end{aligned}
$$

Unless $|\boldsymbol{k}|$ is chosen close to the endpoint $\left(q^{2} / 4-M_{W}^{2}\right)^{1 / 2}$ the numerical difference obtained by replacing the physical limits of the $k^{0}$ integrations by $\pm \infty$ is negligible. We note that the three-momentum distributions shown in Eqs. (66) and

\footnotetext{
${ }^{8}$ This statement is a conjecture. In this context "convergence" for the phase space counter-terms is not meant to be associated with an expansion in $\Gamma_{t}$ or $p_{0}$, but to the convergence in the coefficient multiplying the term linear in $\Gamma_{t}$. All higher-order terms in the nonrelativistic expansion under the phase space integral can contribute to the term linear in $\Gamma_{t}$.
}

(67) are not equal to the physical observable threemomentum distributions, because the exchange of gluons between the top decay and production processes leads to additional non-negligible corrections at NLO [21,22] and NNLO. These "interconnection" effects belong to the electroweak corrections, which are not treated in this work. The NNLO corrections to the three-momentum distribution calculated here are only a first step towards a complete NNLO treatment of the three-momentum distribution. We also want to mention that the three momentum distribution is strictly speaking an ambiguous quantity since the three (as well as the four) momentum of a colored particle is an ambiguous concept. This is in contrast to the total cross section, which describes the rate of color singlet top-quark-top-antiquark events.

We conclude this section with some remarks on the inconsistencies that can arise if the Fadin-Khoze replacement rule " $E \rightarrow E+i \Gamma_{t}$ " is employed at NNLO for the calculation of the top-quark-top-antiquark cross section close to threshold. We emphasize that there is nothing wrong, in principle, in calculating the current correlators for stable top quarks via the Schrödinger equation (19), supplemented afterwards by the replacement $\sqrt{q^{2}}-2 M_{t} \rightarrow \sqrt{q^{2}}-2 M_{t}+i \Gamma_{t}$. This corresponds essentially to the modification

$$
\oint_{n} \frac{|n\rangle\langle n|}{E_{n}-E-i \epsilon} \rightarrow \oiint_{n} \frac{\mid n\langle n|}{E_{n}-E-i \Gamma_{t}}
$$

in the spectral representation of the Green function of Eq. (19) and is equivalent to keeping only the terms $\psi^{\dagger} i \Gamma_{t} \psi$ and $\chi^{\dagger} i \Gamma_{t} \chi$ in the modified version of the NRQCD Lagrangian. In this approach, also the optical theorem remains valid in the form

$$
\operatorname{Im}\left[\mathcal{A}^{\mathrm{v}}\left(q^{2}\right)\right]=6 N_{c} \int \frac{d^{3} \boldsymbol{k}}{(2 \pi)^{3}}|S(\boldsymbol{k})|^{2} \Gamma_{t},
$$

for the vector current correlator, as an example. (For simplicity, we neglect the subtleties of the phase space effects, because they are irrelevant to this discussion.) However, there is a caveat, since it is possible, for the case of zero width, to simplify the form of Eq. (19) in a way that for the stable top-quark case the results remain correct, whereas inconsistencies arise if the results undergo the replacement rule " $E$ $\rightarrow E+i \Gamma_{t}$." Such simplifications, based on the assumption that certain singular terms, which arise during the simplification, can be neglected, have in fact been carried out in Refs. $[17,11,12,13]$. In Refs. $[17,12,34]$ it was shown that the NNLO kinetic energy corrections and the Breit-Fermi potential in Eq. (19), if they are treated as a perturbation to first order, can be rewritten in terms of an energy dependent Coulomb potential $\propto C_{F} \alpha_{s} / \boldsymbol{Q}^{2} \times\left(p_{0}^{2} / M_{t}^{2}\right)$, a Darwin-like constant potential, and a potential $\propto \alpha_{s}^{2} /|\boldsymbol{Q}|$. Neglecting all NNLO corrections except the energy-dependent correc- 
tions to the Coulomb potential, the simplified version of the NNLO Schrödinger equation has the form ${ }^{9}$

$$
\begin{aligned}
& {\left[\frac{\boldsymbol{k}^{2}}{M_{t}}-\frac{p_{0}^{2}}{M_{t}}\right] \tilde{G}\left(\boldsymbol{k}, \boldsymbol{k}^{\prime}\right)+\int \frac{d^{3} \boldsymbol{p}^{\prime}}{(2 \pi)^{3}} \widetilde{V}^{\operatorname{sim}}\left(\boldsymbol{k}-\boldsymbol{p}^{\prime}\right) \widetilde{G}\left(\boldsymbol{p}^{\prime}, \boldsymbol{k}^{\prime}\right)} \\
& \quad=(2 \pi)^{3} \delta^{(3)}\left(\boldsymbol{k}-\boldsymbol{k}^{\prime}\right),
\end{aligned}
$$

where

$$
\widetilde{V}^{\operatorname{sim}}(\boldsymbol{Q})=-\frac{C_{F} 4 \pi \alpha_{s}}{\boldsymbol{Q}^{2}}\left(1+\frac{3 p_{0}^{2}}{2 M_{t}^{2}}\right)
$$

Equation (70) is much easier to solve than the original Schrödinger equation (19). For real energies, and if the corrections from the NNLO terms in Eqs. (19) and (70) are treated as a perturbation to first order only, the results obtained from Eqs. (19) and (70) are indeed equivalent, after a proper renormalization has been carried out. This was in fact the case for which the form of Eq. (70) has been derived in Refs. [17,12,34]. However, Eq. (70) leads to inconsistencies for complex energies. This can be seen from the fact that for the total cross section calculated from Eq. (70), after applying the replacement rule $p_{0}^{2} / M_{t} \rightarrow p_{0}^{2} / M_{t}+i \Gamma_{t}$, the actual form of the optical theorem relation reads

$$
\begin{aligned}
\operatorname{Im} \mathcal{A}^{\mathrm{v}=} & 6 N_{c} \operatorname{Im}\left[\int \frac{d^{3} \boldsymbol{k}}{(2 \pi)^{3}} \int \frac{d^{3} \boldsymbol{k}^{\prime}}{(2 \pi)^{3}} \tilde{G}\left(\boldsymbol{k}, \boldsymbol{k}^{\prime}\right)\right] \\
= & 6 N_{c} \int \frac{d^{3} \boldsymbol{k}}{(2 \pi)^{3}}|S(\boldsymbol{k})|^{2} \Gamma_{t} \\
& -6 N_{c} \int \frac{d^{3} \boldsymbol{k}}{(2 \pi)^{3}} \int \frac{d^{3} \boldsymbol{k}^{\prime}}{(2 \pi)^{3}} S^{*}(\boldsymbol{k}) \\
& \times \operatorname{Im}\left[\widetilde{V}^{\mathrm{sim}}\left(\boldsymbol{k}-\boldsymbol{k}^{\prime}\right)\right] S\left(\boldsymbol{k}^{\prime}\right),
\end{aligned}
$$

rather than Eq. (69). The additional term on the RHS of Eq. (72) originates from the energy dependent Coulomb-type potential in Eq. (70). However, the additional term does not correspond to any physical final state, because it corresponds to an absorption process in the potential. In other words, it is impossible to recover the total cross section from the momentum distribution, if one defines it as the integral over the physical final states represented by the first term on the RHS of Eq. (72). Including also the rest of the NNLO corrections not displayed in Eq. (70), we have checked, with the numerical methods described in the next section, that the size of the second term on the RHS of Eq. (72) is between about 5\% (for $\sqrt{q^{2}}-2 M_{t} \sim 5 \mathrm{GeV}$ ) and $20 \%$ (for $\sqrt{q^{2}}-2 M_{t} \sim$ $-5 \mathrm{GeV}$ ) for the choices of parameters employed in the analysis of Sec. VII. (Similar results can already be obtained by analyzing the known analytic solutions of the nonrelativ-

\footnotetext{
${ }^{9} \mathrm{We}$ emphasize that the neglect of the rest of the NNLO corrections does not affect the validity of the following arguments, because they are independent of the top-quark width after the replacement rule " $E \rightarrow E+i \Gamma_{t}$ "' has been applied.
}

istic Coulomb problem $[42,43,44]$ for a Coulomb potential with a complex coupling.) Thus for the determination of the total top-quark-top-antiquark cross section close to threshold, there is an unacceptable discrepancy between the integrated momentum distribution over physical final states and the absorptive part of the nonrelativistic current correlator. We believe that the size of the second term on the RHS of Eq. (72) should in principle be taken as an estimate for the inherent uncertainties of using the simplified NNLO Schrödinger equation (70) supplemented by the replacement rule of Fadin and Khoze. We have checked, however, that the LHS of Eq. (72) is much closer to the correct result, obtained from the original Schrödinger equation (55), than the integrated momentum distribution. From this point of view the use of the simplified Schrödinger equation (70) might be justified for the total cross section, but is questionable for the momentum distribution.

\section{NUMERICAL IMPLEMENTATION}

In this work we use numerical methods described in Refs. $[8,45,46]$ to determine the vertex functions $S$ and $P$, which are the building blocks for the calculation of the total topquark-top-antiquark production cross section and the three momentum distribution. Because the three- and fourdimensional versions of the vertex functions are related through Eq. (42), it is sufficient to determine the amputated vertex functions $S / G^{f}$ and $P / G^{f}$ from the three-dimensional integral equations (35) and (36). The amputated vertex functions are spherically symmetric and depend only on the modulus of the three momentum $\boldsymbol{k}$. It is therefore possible to reduce Eqs. (35) and (36) to one-dimensional integral equations.

Obviously, when solving Eqs. (35) and (36), the singular behavior of the potentials $\widetilde{V}\left(\boldsymbol{k}, \boldsymbol{p}^{\prime}\right)$ and $\tilde{V}_{c}^{\mathrm{LO}}\left(\boldsymbol{k}, \boldsymbol{p}^{\prime}\right)$ for $\boldsymbol{p}^{\prime}$ $\rightarrow \boldsymbol{k}$ requires special treatment. To avoid numerical problems we rewrite the integral equations for the amputated vertex functions as

$$
\begin{aligned}
\mathcal{K}^{\vee}(\boldsymbol{k}) \equiv & \frac{S(\boldsymbol{k})}{G^{f}(\boldsymbol{k})} \\
= & 1+\frac{\boldsymbol{k}^{2}}{6 M_{t}^{2}}-\int \frac{d^{3} \boldsymbol{p}^{\prime}}{(2 \pi)^{3}} \tilde{V}\left(\boldsymbol{k}, \boldsymbol{p}^{\prime}\right) G^{f}(\boldsymbol{k}) \mathcal{K}^{\vee}\left(\boldsymbol{p}^{\prime}\right) \\
= & 1+\frac{\boldsymbol{k}^{2}}{6 M_{t}^{2}}-\int \frac{d^{3} \boldsymbol{p}^{\prime}}{(2 \pi)^{3}} \tilde{V}\left(\boldsymbol{k}, \boldsymbol{p}^{\prime}\right) G^{f}(\boldsymbol{k})\left[\mathcal{K}^{\vee}\left(\boldsymbol{p}^{\prime}\right)\right. \\
& \left.-\mathcal{K}^{\vee}(\boldsymbol{k})\right]-\mathcal{B}^{\vee}(\boldsymbol{k}) \mathcal{K}^{\vee}(\boldsymbol{k}), \\
\mathcal{K}^{a}(\boldsymbol{k}) \equiv & \frac{P(\boldsymbol{k})}{G^{f}(\boldsymbol{k})} \\
= & 1-\int \frac{d^{3} \boldsymbol{p}^{\prime}}{(2 \pi)^{3}} \frac{\boldsymbol{k} \boldsymbol{p}^{\prime}}{k^{2}} \tilde{V}_{c}^{\mathrm{LO}}\left(\boldsymbol{k}, \boldsymbol{p}^{\prime}\right) G^{f}(\boldsymbol{k}) \mathcal{K}^{a}\left(\boldsymbol{p}^{\prime}\right) \\
= & 1-\int \frac{d^{3} \boldsymbol{p}^{\prime}}{(2 \pi)^{3}} \tilde{V}_{c}^{\mathrm{LO}}\left(\boldsymbol{k}, \boldsymbol{p}^{\prime}\right) G^{f}(\boldsymbol{k})\left(\frac{\boldsymbol{k} \boldsymbol{p}^{\prime}}{k^{2}} \mathcal{K}^{a}\left(\boldsymbol{p}^{\prime}\right)-\mathcal{K}^{a}(\boldsymbol{k})\right) \\
& -\mathcal{B}^{a}(\boldsymbol{k}) \mathcal{K}^{a}(\boldsymbol{k}),
\end{aligned}
$$


where $\mathcal{B}^{\vee}$ and $\mathcal{B}^{a}$ are defined as

$$
\begin{aligned}
\mathcal{B}^{\vee}(\boldsymbol{k}) & \equiv \int \frac{d^{3} \boldsymbol{p}^{\prime}}{(2 \pi)^{3}} \tilde{V}\left(\boldsymbol{k}, \boldsymbol{p}^{\prime}\right) G^{f}(\boldsymbol{k}), \\
\mathcal{B}^{a}(\boldsymbol{k}) & \equiv \int \frac{d^{3} \boldsymbol{p}^{\prime}}{(2 \pi)^{3}} \widetilde{V}_{c}^{\mathrm{LO}}\left(\boldsymbol{k}, \boldsymbol{p}^{\prime}\right) G^{f}(\boldsymbol{k}) .
\end{aligned}
$$

As mentioned above, both $\mathcal{K}^{\vee}$ and $\mathcal{K}^{a}$ depend only on the modulus of the three momentum. The angular dependence of the integrand in Eqs. (73) and (74) (including $\mathcal{B}^{\vee}$ and $\mathcal{B}^{a}$ ) is only coming from the potentials and the dot product $\boldsymbol{k} \boldsymbol{p}^{\prime}$. The angular integration can be carried out analytically. The remaining one-dimensional integral equations are then solved numerically by discretization: the integrals $\int d p^{\prime} \quad\left(p^{\prime} \equiv\left|\boldsymbol{p}^{\prime}\right|\right)$ are transformed into sums $\Sigma_{i}$ over a fixed set of momenta $p^{\prime i}$, and the integral equations for $\mathcal{K}^{\mathrm{V}}(\boldsymbol{k})$ and $\mathcal{K}^{a}(\boldsymbol{k})$ are each reduced to a system of linear equations, where the same set of momenta has to be used for the $k^{i}{ }^{10}$ The resulting (complex) matrices are then inverted numerically to give the amputated vertex functions $\mathcal{K}^{\mathrm{V}}$ and $\mathcal{K}^{a}$ for the momenta $k^{i}$.

In practice it turned out that the use of the Gaussian quadrature formulas is very efficient for the discretization. A surprisingly small number of points (of the order of 100) already leads to a high numerical accuracy. In addition, integrals were split into two (or more) parts and a suitable transformation of integration variables was applied wherever needed. It should also be noted that the finite width of the top quark is essential for the numerical stability of the method. It makes potentially dangerous denominators in the integrands of Eqs. (73) and (74), which originate from the free Green function, well behaved [compare Eqs. (37) and (56)]. Clearly, the UV regularization by a momentum cutoff as discussed in Sec. IV is most naturally implemented in our numerical approach. It is sufficient to choose the momenta of the (Gauss-Legendre) grid to be limited by the value of the cutoff. Such a cutoff is, in principle, not needed in the case of pure Coulomb potentials. There the solution of the integral equations is possible without any cutoff and would correspond to a different regularization scheme with different short-distance coefficients. However, the potentials $\widetilde{V}_{\mathrm{BF}}$, $\widetilde{V}_{\mathrm{NA}}$ and the kinematic corrections introduced at NNLO require a UV regularization already for purely numerical reasons, which can be seen from naive power counting in Eq. (73).

\section{A FIRST ANALYSIS IN THE POLE MASS SCHEME}

In this section we carry out a first brief analysis of the total cross sections $Q_{t}^{2} R^{\vee}$ and $R^{a}$ and their three-momentum distributions in the pole mass scheme. We do this even

\footnotetext{
${ }^{10}$ At this point the subtraction carried out in Eqs. (73) and (74) becomes crucial. Without it the singularities in the potentials for $p^{\prime} \rightarrow k$ would be manifest for $p^{i^{\prime}}=k^{i}$, even in the case of integrable singularities.
}

though it is known that in the pole mass scheme there are uncomfortably large NNLO corrections in the location of the $1 S$ peak position as well as in the normalization of the total vector-current-induced cross section $R^{\vee}[11-13]$. We will show in Sec. VIII that these large corrections are a consequence of the pole mass scheme, and that the pole mass definition has to be abandoned as far as a precise extraction of a top-quark mass from experimental data is concerned. Nevertheless, the pole mass is a well defined quantity in the framework of perturbation theory $[47,48]$ and, despite all its problems at larger orders of perturbation theory, remains a very convenient mass parameter to use for the formulation of (P)NRQCD and for calculations of the cross section. Thus a brief analysis in the pole mass scheme serves as a reference point with which results obtained with different approaches can be compared and from which we can visualize in which way alternative top quark mass definitions can improve the situation and in which way they cannot.

In Fig. 3 the total vector-current-induced cross section $Q_{t}^{2} R^{\vee}$ is displayed for $344 \mathrm{GeV}<\sqrt{q^{2}}<352 \mathrm{GeV}$ at $\mathrm{LO}$ (dotted lines), NLO (dashed lines), and NNLO (solid lines). The LO curves are determined from Eq. (55), excluding all relativistic corrections and taking into account only the $\mathcal{O}\left(\alpha_{s}\right)$ contribution to the Coulomb potential and setting $C^{\vee}=1$. In addition, the NLO curves also include the NLO corrections to $C^{\mathrm{v}}$ and the $\mathcal{O}\left(\alpha_{s}^{2}\right)$ contributions of the Coulomb potential. At NNLO all contributions mentioned in this paper are included. Only at NNLO have we taken into account the phase space counterterms displayed in Eqs. (64) and (65). In all figures shown in this section the top-quark width is chosen as $\Gamma_{t}=1.43 \mathrm{GeV}$ and the top-quark pole mass as $M_{t}$ $=175 \mathrm{GeV}$. Figure 3(a) displays the dependence on the renormalization scale for $\mu=15,30$, and $60 \mathrm{GeV}$ for $\alpha_{s}\left(M_{Z}\right)=0.118, \Lambda=175 \mathrm{GeV}$. For $\sqrt{q^{2}} \leqslant 347 \mathrm{GeV}, \mu=15$, 30 , and $60 \mathrm{GeV}$ corresponds to the upper, middle, and lower curves, respectively. We note that in Refs. [11-13] the renormalization scale governing the strong coupling of the potentials in the Schrödinger equation (19) has been chosen between 50 and $100 \mathrm{GeV}$. Regarding the fact that the inverse Bohr radius $\sim M_{t} \alpha_{s}$ is the scale that governs the cross section at NNLO, the natural renormalization scale is of order $30 \mathrm{GeV}$. This causes logarithms of the ratio $\Lambda / \mu \sim 1 / \alpha_{s}$ in the vector current correlator (see the Appendix), which, however, are connected only to the running of the strong coupling. As has already been demonstrated in previous publications on the NNLO corrections [11-13], the position of the $1 S$ peak varies considerably at the different orders of approximation and for the different choices of the renormalization scale $\mu$. (For explicit numbers see Table I.) It is also evident that the normalization of the total cross section is subject to large corrections. We see that, in general, the NNLO corrections to the normalization are of order $20 \%$ and as large as the NLO ones. Further, the dependence on the renormalization scale is even larger at NNLO than at NLO. In Fig. 3(b) the dependence of $Q_{t}^{2} R^{\mathrm{V}}$ on the choice of the cutoff $\Lambda$ is shown for $\alpha_{s}\left(M_{Z}\right)=0.118, \mu=30 \mathrm{GeV}$ and $\Lambda=90,175$, and $350 \mathrm{GeV}$. For LO and NNLO $\Lambda=90,175$, and $350 \mathrm{GeV}$ correspond to the lower, middle, and upper 

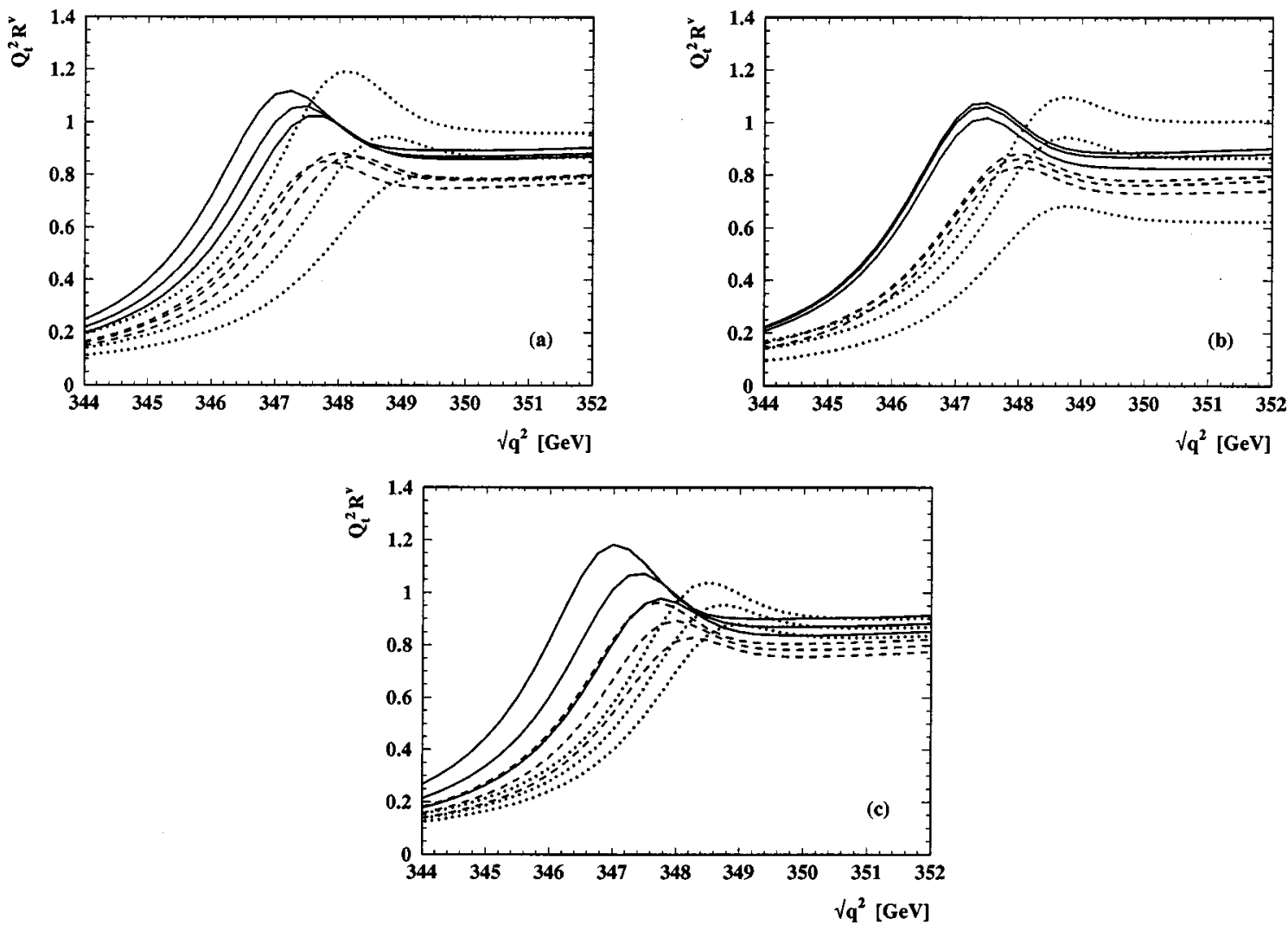

FIG. 3. The total vector-current-induced cross section $Q_{t}^{2} R^{\vee}$ for center-of-mass energies $344 \mathrm{GeV}<\sqrt{q^{2}}<352 \mathrm{GeV}$ in the pole mass scheme. The dependence on the renormalization scale $\mu(\mathrm{a})$, on the cutoff $\Lambda(\mathrm{b})$, and on $\alpha_{s}\left(M_{Z}\right)(\mathrm{c})$ is displayed. More details and the choice of parameters are given in the text.

curves, respectively. For NLO $\Lambda=90,175$, and $350 \mathrm{GeV}$ correspond to the lower, upper, and middle curve, respectively. Whereas the dependence of the LO cross section on the choice of $\Lambda$ is quite dramatic, because at LO there is no short-distance correction that could compensate for a variation in $\Lambda$, the variations at NLO and NNLO are significantly smaller (or order $5-10 \%$ ). However, there is again no reduc-

TABLE I. LO, NLO, and NNLO contributions to the peak position of the total vector current induced cross section $R^{\vee}$ in GeV in the pole mass scheme for $M_{t}=175 \mathrm{GeV}, \Gamma_{t}=1.43 \mathrm{GeV}$, $\alpha_{s}\left(M_{Z}\right)=0.113,0.118$, and 0.123 , and $\mu=15,30$, and $60 \mathrm{GeV}$, respectively. For the strong coupling two-loop running has been employed. The results are insensitive to the choice of the cutoff scale $\Lambda \sim M_{t}$.

\begin{tabular}{ccccccc}
\hline \hline$\mu[\mathrm{GeV}] \alpha_{s}\left(M_{Z}\right)$ & $\delta M_{\text {peak }}^{\mathrm{LO}}$ & $\delta M_{\text {peak }}^{\mathrm{NLO}}$ & $\delta M_{\text {peak }}^{\mathrm{NNLO}, \beta_{0}}$ & $\delta M_{\text {peak }}^{\mathrm{NNLO} \text {,est }}$ & $\delta M_{\text {peak }}$ \\
\hline 15 & 0.113 & 1.60 & 0.28 & 0.20 & 0.32 & 2.40 \\
30 & & 1.02 & 0.70 & 0.27 & 0.24 & 2.23 \\
60 & & 0.35 & 1.18 & 0.36 & 0.18 & 2.07 \\
15 & 0.118 & 1.89 & 0.31 & 0.24 & 0.37 & 2.80 \\
30 & & 1.26 & 0.75 & 0.31 & 0.27 & 2.58 \\
60 & & 0.69 & 1.09 & 0.40 & 0.20 & 2.39 \\
15 & 0.123 & 2.17 & 0.33 & 0.29 & 0.43 & 3.23 \\
30 & & 1.49 & 0.81 & 0.36 & 0.31 & 2.96 \\
60 & & 0.92 & 1.12 & 0.45 & 0.23 & 2.72 \\
\hline \hline
\end{tabular}

tion of the variation from NLO to NNLO. The variation of the cross section with respect to $\Lambda$ is small compared with the variation with respect to the renormalization scale $\mu$ for center-of-mass energies closer and below the peak. Figure 3(c) displays the dependence of $Q_{t}^{2} R^{\mathrm{v}}$ on the choice of $\alpha_{s}\left(M_{Z}\right)$ for $\alpha_{s}\left(M_{z}\right)=0.113,0.118$, and 0.123 and $\Lambda=175$ $\mathrm{GeV}, \mu=30 \mathrm{GeV}$. As for the variations in the renormalization scale we see that the position of the peak depends considerably on the choice of $\alpha_{s}$. We observe a strong positive correlation between the choice of $M_{t}$ and $\alpha_{s}\left(M_{Z}\right)$. Because the peak in the total cross section is the most pronounced feature of the total cross section, its behavior directly reflects the quality of a top-quark mass extraction from experimental data. Thus, if one would like to fit the pole mass to data for the cross section line shape from a threshold scan, one finds a strong positive correlation between the pole mass and the strong coupling [4] and a strong dependence of $M_{t}$ on the choice of the renormalization scale leading to quite large theoretical uncertainties in the pole mass measurements.

In Figs. 4(a)-4(c) the total axial-vector-current-induced cross section $R^{a}$ is displayed for the same input parameters as in Fig. 3. Figure 4(a) shows the dependence on the renormalization scale for $\mu=15$ (solid line), 30 (dashed line), and $60 \mathrm{GeV}$ (dotted line). Figure 4(b) exhibits the dependence of the cutoff for $\Lambda=90$ (solid line), 175 (dashed line), and 350 $\mathrm{GeV}$ (dotted line). Figure 4(c) shows $R^{a}$ for $\alpha_{s}\left(M_{z}\right)=0.113$ (solid line), 0.118 (dashed line), and 0.123 (dotted line). We 

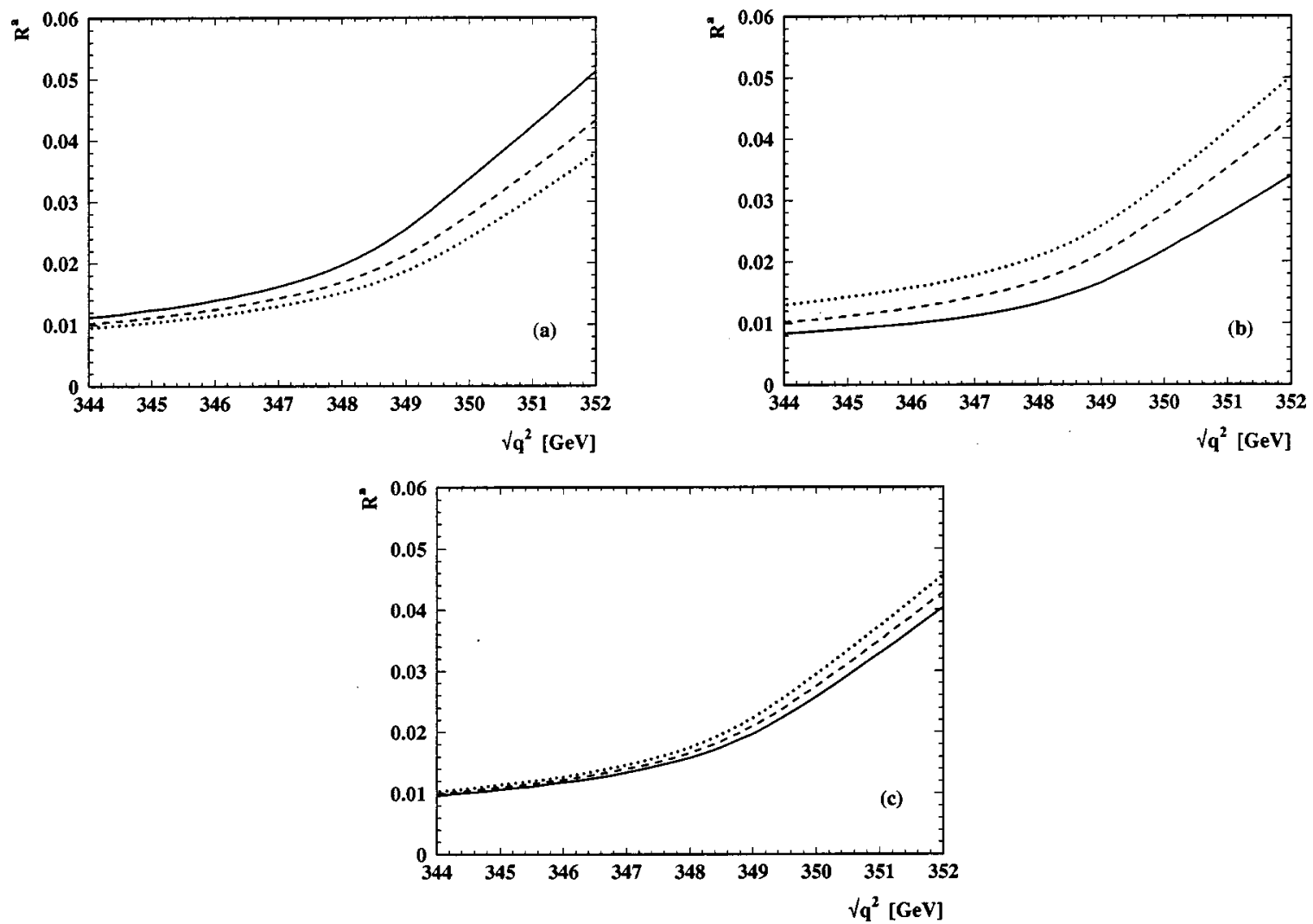

FIG. 4. The total axial-vector-current induced cross section $R^{a}$ for center-of-mass energies $344 \mathrm{GeV}<\sqrt{q^{2}}<352 \mathrm{GeV}$ in the pole mass scheme. The dependence on the renormalization scale $\mu(\mathrm{a})$, on the cutoff $\Lambda(\mathrm{b})$, and on $\alpha_{s}\left(M_{Z}\right)$ (c) is displayed. More details and the choice of parameters are given in the text.

observe that due to the $\mathrm{v}$ suppression of the axial-vector currents no peak-line enhancement as in the vector current case is visible. The variations of $R^{a}$ with respect to the renormalization scale and the cutoff are quite large, because $R^{a}$ has only been determined at leading order, and the shortdistance coefficient $C^{a}$ does not contain any corrections that could compensate for the variations. We note that the NLO corrections to $R^{a}$ can be implemented in the same way as for $R^{\mathrm{v}}$. They are, however, beyond NNLO and have therefore not been included into our analysis. From the phenomenological point of view the next-to-leading order corrections are irrelevant if one takes into account the small normalization of $R^{a}$ compared to $R^{\mathrm{v}}$.

In Figs. 5(a)-5(c) the LO (dotted lines), NLO (dashed line), and NNLO (solid lines) top-quark-top-antiquark vector-current-induced three momentum distribution $Q_{t}^{2} d R^{\mathrm{v}} / d\left|\boldsymbol{k}_{t}\right|$ is shown for $0<\left|\boldsymbol{k}_{t}\right|<60 \mathrm{GeV}$ and both for center-of-mass energies exactly on top of the visible peak, $\sqrt{q^{2}}=M_{\text {peak }}$, and for $\sqrt{q^{2}}=M_{\text {peak }}+5 \mathrm{GeV}$. The input parameters have been chosen as in Figs. 3. Figure 5(a) shows the distributions for $\mu=15$ and $60 \mathrm{GeV}$. At LO and NNLO $\mu=15 \mathrm{GeV}$ corresponds to the upper curves below the peak and $\mu=60 \mathrm{GeV}$ to the lower curves. At NLO $\mu=60 \mathrm{GeV}$ corresponds to the higher peak and $\mu=15 \mathrm{GeV}$ to the lower. Figure 5(b) displays the dependence of the distributions on the cutoff for $\Lambda=90$ (lower curves) and $350 \mathrm{GeV}$ (upper curves), and Fig. 5(c) exhibits the dependence of the distributions on the strong coupling for $\alpha_{s}\left(M_{Z}\right)=0.113$ and
0.123. Below the peak, the larger value of $\alpha_{s}\left(M_{Z}\right)$ always corresponds to the upper curve. As for the total cross section, we observe a strong dependence of the normalization of the distributions on the renormalization scale and the cutoff. For $\sqrt{q^{2}}=M_{\text {peak }}+5 \mathrm{GeV}$, the dependence of the peak position on the renormalization scale is particularly strong. ${ }^{11}$

Finally, in Figs. 6(a)-6(c) the top-quark-top-antiquark axial-vector-current-induced three momentum distribution $d R^{a} / d\left|\boldsymbol{k}_{t}\right|$ is shown for $0<\left|\boldsymbol{k}_{t}\right|<60 \mathrm{GeV}$ and for center-ofmass energies exactly on top of the visible peak, $\sqrt{q^{2}}$ $=M_{\text {peak }}$, and for $\sqrt{q^{2}}=M_{\text {peak }}+5 \mathrm{GeV}$. The input parameters have been chosen as before. Figure 6(a) shows the distributions for $\mu=15$ (solid curves), 30 (dashed curves), and 60 $\mathrm{GeV}$ (dotted curves). Figure 6(b) displays the dependence of the distributions on the cutoff for $\Lambda=90$ (solid curves), 175 (dashed curves), and $350 \mathrm{GeV}$ (dotted curves), and Fig. 6(c) exhibits the dependence of the distributions on the strong coupling for $\alpha_{s}\left(M_{Z}\right)=0.113$ (solid curves), 0.118 (dashed curves), and 0.123 (dotted curves). As expected, the momentum distribution is strongly suppressed for smaller center-ofmass energies. The variations of the normalization of the distributions are comparable to the variations of the normalization of the total cross sections.

\footnotetext{
${ }^{11}$ The peak position is always located approximately at $\left|\boldsymbol{k}_{t}\right| \approx\left(p_{0}^{4}\right.$ $\left.+M_{t}^{2} \Gamma_{t}^{2}\right)^{1 / 4}$, which can be regarded as the effective three momentum of the top quarks.
} 

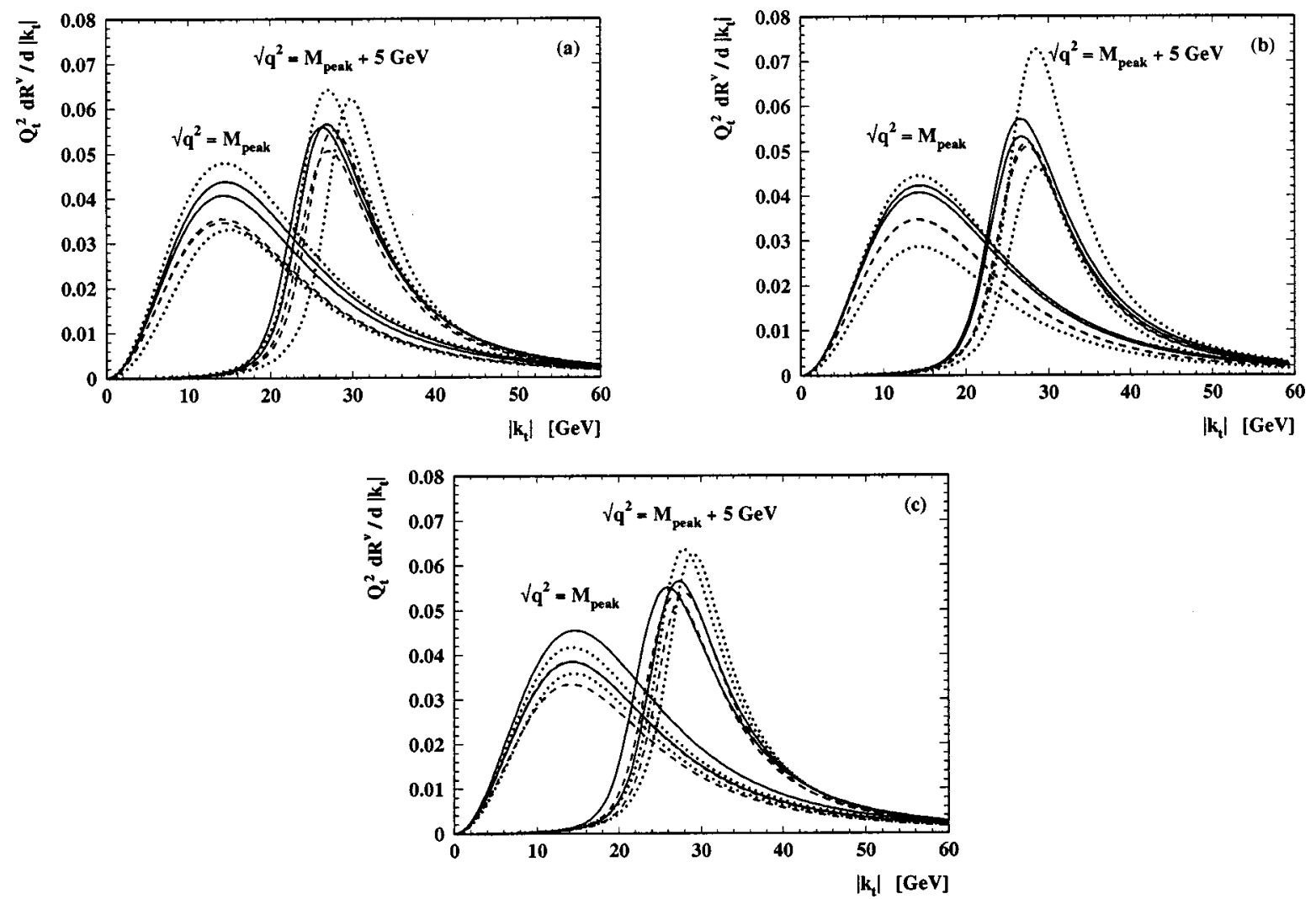

FIG. 5. The three-momentum distribution of the vector-current-induced cross section $Q_{t}^{2} R^{\vee}$ for center-of-mass energies $\sqrt{q^{2}}=M_{\text {peak }}$ and $M_{\text {peak }}+5 \mathrm{GeV}$ in the pole mass scheme. The dependence on the renormalization scale $\mu$ (a), on the cutoff $\Lambda$ (b), and on $\alpha_{s}\left(M_{Z}\right)$ (c) is displayed. More details and the choice of parameters are given in the text.

\section{THEORETICAL UNCERTAINTIES}

In the previous section we have seen that the location of the peak position and the normalization of the vectorcurrent-induced total cross section, as well as of its threemomentum distribution, receive large NNLO corrections in the pole mass scheme. In the following two subsections we discuss the origin of the large corrections in the vectorcurrent-induced total cross section. For the peak position of the total cross section we propose a solution, which allows for a considerable stabilization.

\section{A. The $1 S$ top-quark mass and the peak position}

From past experience in the theoretical description of $B$-meson decays $[49,50]$, it is well known that the pole mass, defined as the pole of the perturbative quark propagator, although infrared-finite to all orders in perturbation theory $[47,48]$, is a concept that is ambiguous to an amount of order $\Lambda_{\mathrm{QCD}}$. This might be a reflection of the fact that the perturbative quark pole does not exist in reality because of confinement. Within the framework of perturbation theory this ambiguity is caused by an $n$-factorial increase of the coefficients in the perturbative relation between the pole mass and a short-distance mass such as $\overline{\mathrm{MS}}$. The large corrections are caused by an increasing infrared sensitivity of the perturbative coefficients for large orders. It has also been shown that the large top width does not lead to a suppression of these large corrections [51]. From this point of view the unstable behavior of the peak position in the total cross section is not unexpected and it would be quite appealing conceptually to conclude that the use of a short-distance mass instead of the pole definition would cure the problem.

In fact, it has been demonstrated in Refs. [52,53] that the dominant source of infrared sensitivity in the Green function of the Schrödinger equation (19) comes from the terms in the static energy $2 M_{t}+V(\boldsymbol{r})$. Whereas the rest (pole) mass energy $2 M_{t}$ and the potential energy $V$ (which has traditionally been calculated in the pole mass scheme [26-29]) are individually ambiguous to an amount of order $\Lambda_{\mathrm{QCD}}[49,50,54]$, the sum of both is not $[52,53]$. This shows that quantities such as spectra or the total cross section calculated from Eq. (19) are much less sensitive to infrared momenta than the pole mass itself, rendering it an irrelevant mass parameter. Thus, any sensible mass definition used to parametrize the top-quark-top-antiquark cross section close to threshold should have no ambiguity of order $\Lambda_{\mathrm{QCD}}$, i.e., it should be a short-distance mass.

However, we emphasize that, in practice, large corrections at lower orders in perturbation theory in the pole scheme do not necessarily come from the ambiguity in the pole mass. This is because the cancellations of infrared sensitive contributions in static and rest mass energy is a phenomenon that is relevant in large orders where the corresponding series are dominated by the most infrared sensitive 

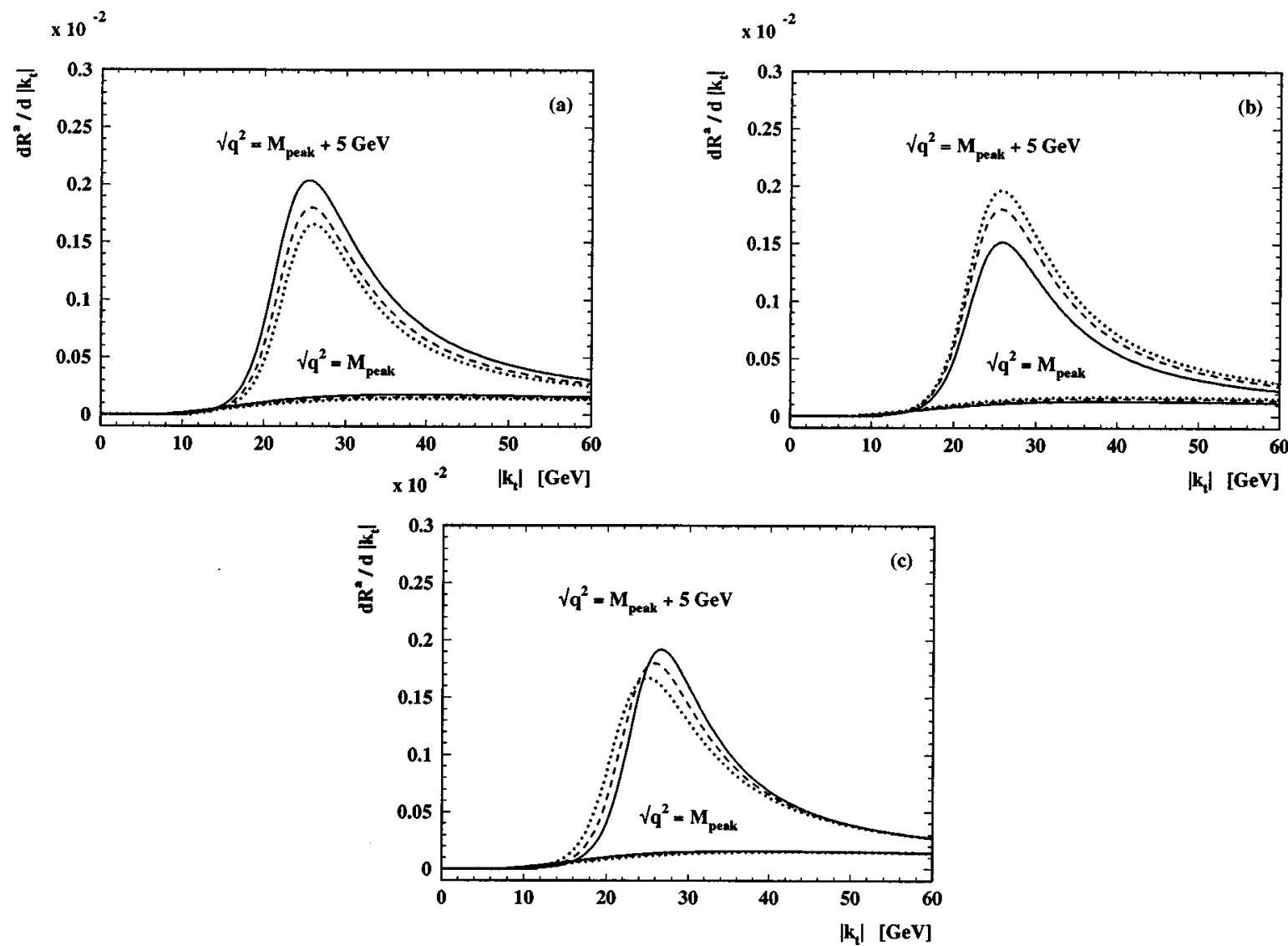

FIG. 6. The three-momentum distribution of the axial-vector-current-induced cross section $R^{a}$ for center-of-mass energies $\sqrt{q^{2}}=M_{\text {peak }}$ and $M_{\text {peak }}+5 \mathrm{GeV}$ in the pole mass scheme. The dependence on the renormalization scale $\mu(\mathrm{a})$, on the cutoff $\Lambda$ (b), and on $\alpha_{s}\left(M_{Z}\right)(\mathrm{c})$ is displayed. More details and the choice of parameters are given in the text.

contributions in the loop integrals. Thus, the large corrections in perturbation theory at low orders could very well come from scales which are not infrared. To get a clearer picture for the case of the peak position in the total cross section, let us have a look at the size of the individual corrections to the peak position. In Table I we have displayed the LO, NLO, and NNLO contributions to the peak position with respect to the pole rest mass energy

$$
\begin{aligned}
M_{\text {peak }} & =2 M_{t}-\delta M_{\text {peak }}^{\mathrm{LO}}-\delta M_{\text {peak }}^{\mathrm{NLO}}-\delta M_{\text {peak }}^{\mathrm{NNLO}, \beta_{0}}-\delta M_{\text {peak }}^{\mathrm{NNL}, \text { rest }} \\
& =2 M_{t}-\delta M_{\text {peak }}
\end{aligned}
$$

for $M_{t}=175 \mathrm{GeV}, \Gamma_{t}=1.43 \mathrm{GeV}, \alpha_{s}\left(M_{Z}\right)=0.113,0.118$, and 0.123 , and $\mu=15,30$, and $60 \mathrm{GeV}$. At NNLO we have separated from the rest the contributions with the highest power of $\beta_{0}$, which represent the contributions most sensitive to infrared momenta. ${ }^{12}$ All the large- $\beta_{0}$ terms originate

\footnotetext{
${ }^{12}$ These contributions are determined by using the replacement rule $n_{f} \rightarrow-\frac{3}{2} \beta_{0}$ for the terms with the highest power of $n_{f}$, where $n_{f}$ is the number of light quark species. This method is called "naive non-Abelianization" and accounts for the most infrared sensitive contributions in perturbative series relating the pole mass to a short-distance mass [55]. The result of this replacement is referred to as the "large- $\beta_{0}$ " limit.
}

from the Coulomb potential $V_{c}$, Eq. (22). From the numbers presented in Table I we see that depending on the choice of the renormalization scale the large- $\beta_{0}$ contributions at NNLO contribute between about 30 and $60 \%$ to the total NNLO corrections to the peak position. Thus at NNLO the large shift in the peak position consists to approximately equal parts of corrections very sensitive to infrared momenta and corrections coming from subleading infrared terms and relativistic corrections. From this we can conclude that using some unspecified short-distance mass definition instead of the pole mass does not necessarily lead to smaller NNLO corrections to the peak position because the most infrared sensitive terms are not yet dominating at NNLO. For the same reason, we cannot conclude that some unspecified short-distance mass definition necessarily leads to a significantly reduced renormalization scale dependence of the NNLO peak position or to a smaller correlation between the peak position and the strong coupling. Thus, the question of which mass definition one should use is not only a conceptual issue, but also a practical one.

We formulate two requirements for a proper top mass definition for the total cross section close to threshold: (A) it must be a short-distance mass, and (B) it must lead to a considerable stabilization of the peak location with respect to the order of approximation used and also to variations of parameters such as the strong coupling or the renormaliza- 
tion scale. Requirement (A) reflects the necessity that, if a top mass determination at the linear collider with uncertainties of $200 \mathrm{MeV}$ or even better is intended, the corresponding mass parameter must be free of intrinsic ambiguities of order $\Lambda_{\mathrm{QCD}}$. In addition, only a short-distance mass can be reliably related to the $\overline{\mathrm{MS}}$ top-quark mass, which is the preferred mass parameter used in calculations at high energies and for top-quark corrections to electroweak precision observables. Requirement (B) ensures that the mass parameter can be extracted from experimental data with small systematic uncertainties.

The mass that seems to be most appropriate to us to fulfil this task, because it is closely related to the peak position in the vector-current-induced cross section, is what we call the $1 S$ mass, $M_{1 S}$. The $1 S$ mass is defined as half the perturbative mass of the fictitious toponium $1{ }^{3} S_{1}$ ground state, where the top quark is assumed to be stable. Expressed in terms of the pole mass, the $1 S$ mass reads $\left[a_{s}=\alpha_{s}(\mu)\right]$ $[56,57]$

$$
\begin{aligned}
M_{1 S}= & M_{t}-\epsilon \frac{M_{t} C_{F}^{2} a_{s}^{2}}{8}-\epsilon^{2} \frac{M_{t} C_{F}^{2} a_{s}^{2}}{8}\left(\frac{a_{s}}{\pi}\right)\left[\beta_{0}(L+1)+\frac{a_{1}}{2}\right] \\
& -\epsilon^{3} \frac{M_{t} C_{F}^{2} a_{s}^{2}}{8}\left(\frac{a_{s}}{\pi}\right)^{2}\left[\beta_{0}^{2}\left(\frac{3}{4} L^{2}+L+\frac{\zeta_{3}}{2}+\frac{\pi^{2}}{24}+\frac{1}{4}\right)\right. \\
& +\beta_{0} \frac{a_{1}}{2}\left(\frac{3}{2} L+1\right)+\frac{\beta_{1}}{4}(L+1)+\frac{a_{1}^{2}}{16}+\frac{a_{2}}{8} \\
& \left.+\left(C_{A}-\frac{C_{F}}{48}\right) C_{F} \pi^{2}\right]
\end{aligned}
$$

where

$$
L \equiv \ln \left(\frac{\mu}{C_{F} a_{s} M_{t}}\right)
$$

and the contributions at LO, NLO, and NNLO are labeled by the powers $\epsilon, \epsilon^{2}$, and $\epsilon^{3}$, respectively, of the auxiliary parameter $\epsilon=1$. In general, the electroweak corrections not calculated in this work can lead to further corrections in Eq. (78). We note that $2 M_{1 S}$ is not equal to the actual peak position visible in the vector-current-induced total cross section because the top-quark width leads to an additional shift of the peak by about $+200 \mathrm{MeV}$. ${ }^{13}$ In principle it would also be possible to define a mass that would be equal to half the actual visible peak position. Except for additional corrections coming from the top-quark width, this would also require the inclusion of an additional shift coming from the axial-vector-

\footnotetext{
${ }^{13}$ The difference between $2 M_{1 S}$ and the peak location of the vector-current-induced total cross section is proportional to $\Gamma_{t}$ times a function of $\Gamma_{t} /\left(M_{t} \alpha_{s}^{2}\right)$. For $\Gamma_{t} \ll M_{t} \alpha_{s}^{2}$ the difference $M_{\text {peak }}$ $-2 M_{1 S}$ is proportional to $\Gamma_{t}^{4} /\left(M_{t} C_{F}^{2} \alpha_{s}^{2}\right)^{3}$. The size of the difference between $2 M_{1 S}$ and the peak location of about $+200 \mathrm{MeV}$ can be seen in Fig. 7.
}

induced cross section. Such a definition would, however, not necessarily be more useful, since the experimentally measurable line shape of the total cross section at a future $e^{+} e^{-}$or $\mu^{+} \mu^{-}$collider will be distorted by initial state radiation and beamstrahlung. In the case of a muon collider these effects lead to an additional shift and in the case of the $e^{+} e^{-}$linear collider even to the disappearance of the peak [4]. Thus one has to consider the $1 S$ mass, like the $\overline{\mathrm{MS}}$ mass, as a fictitious mass parameter, which to NNLO is defined through the perturbative series given in Eq. (78). Nevertheless, twice the $1 S$ mass is quite close to the peak location and, by construction, leads to a considerable reduction of the variation of the peak position with respect to the order of approximation, the strong coupling and the renormalization scale.

To show that $M_{1 S}$ is indeed a short-distance mass we recall that the static energy $2 M_{t}+V_{c}(\boldsymbol{r})$ represents the dominant infrared sensitive contribution in the Schrödinger equation (19). The difference between $M_{1 S}$ and the pole mass is ${ }^{14}$

$$
M_{1 S}-M_{t}=\frac{1}{2} \int \frac{d^{3} \boldsymbol{p}}{(2 \pi)^{3}} \frac{d^{3} \boldsymbol{q}}{(2 \pi)^{3}} \widetilde{\Phi}_{1 S}^{*}(\boldsymbol{p}) \mathcal{H}(\boldsymbol{p}, \boldsymbol{q}) \widetilde{\Phi}_{1 S}(\boldsymbol{q}),
$$

where $\mathcal{H}$ is the Hamiltonian of Eq. (19) and $\widetilde{\Phi}_{1 S}$ the normalized wave function of the $1 S$ state in momentum space representation. The dominant infrared sensitive contribution in relation (80) reads

$$
\left(M_{1 S}-M_{t}\right)^{\mathrm{IR}} \sim \frac{1}{2} \int \frac{d^{3} \boldsymbol{p}}{(2 \pi)^{3}} \frac{d^{3} \boldsymbol{q}}{(2 \pi)^{3}} \tilde{\Phi}_{1 S}^{*}(\boldsymbol{p}) \tilde{V}_{c}(\boldsymbol{p}-\boldsymbol{q}) \tilde{\Phi}_{1 S}(\boldsymbol{q}) .
$$

Because the infrared region in Eq. (81) is given by $|\boldsymbol{p}-\boldsymbol{q}|$ $<\mu_{f}$, where $\mu_{f}$ is much smaller than the inverse Bohr radius $\sim M_{t} \alpha_{s}$, the characteristic scale governing the dynamics described by the wave function, we can simplify relation $(81)$,

$$
\begin{aligned}
\left(M_{1 S}-M_{t}\right)^{\mathrm{IR}} \sim & \frac{1}{2} \int^{|\boldsymbol{p}-\boldsymbol{q}|<\mu_{f}} \frac{d^{3} \boldsymbol{p}}{(2 \pi)^{3}} \frac{d^{3} \boldsymbol{q}}{(2 \pi)^{3}} \\
& \times\left|\tilde{\Phi}_{1 S}(\boldsymbol{p})\right|^{2} \tilde{V}_{c}(\boldsymbol{p}-\boldsymbol{q}) \\
& \sim \frac{1}{2} \int^{|\boldsymbol{q}|<\mu_{f}} \frac{d^{3} \boldsymbol{q}}{(2 \pi)^{3}} \tilde{V}_{c}(\boldsymbol{q}) .
\end{aligned}
$$

\footnotetext{
${ }^{14}$ Strictly speaking, the simple form of Eq. (80) is true only up to NNLO because of retardation effects, which set in at $\mathrm{N}^{3} \mathrm{LO}$. Thus, in general, there would also be a nontrivial integration over time components. The form of our proof also depends on the assumption that the static potential is an infrared finite quantity. That this is most probably not the case was already pointed out some time ago in Ref. [58], because the perturbative static potential might become sensitive to scales below the inverse Bohr radius at $\mathcal{O}\left(\alpha_{s}^{4}\right)$. Some contributions at $\mathcal{O}\left(\alpha_{s}^{4}\right)$ have recently been calculated in Ref. [59]. Up to $\mathcal{O}\left(\alpha_{s}^{3}\right)$, i.e., NNLO in the non-relativistic expansion, the perturbative potential has been proven to be finite by complete calculations $[28,29]$. Because $M_{1 S}$ is defined as a physical quantity this would not affect the final conclusion that it is a short-distance mass, but it would change the form of the proof considerably.
} 

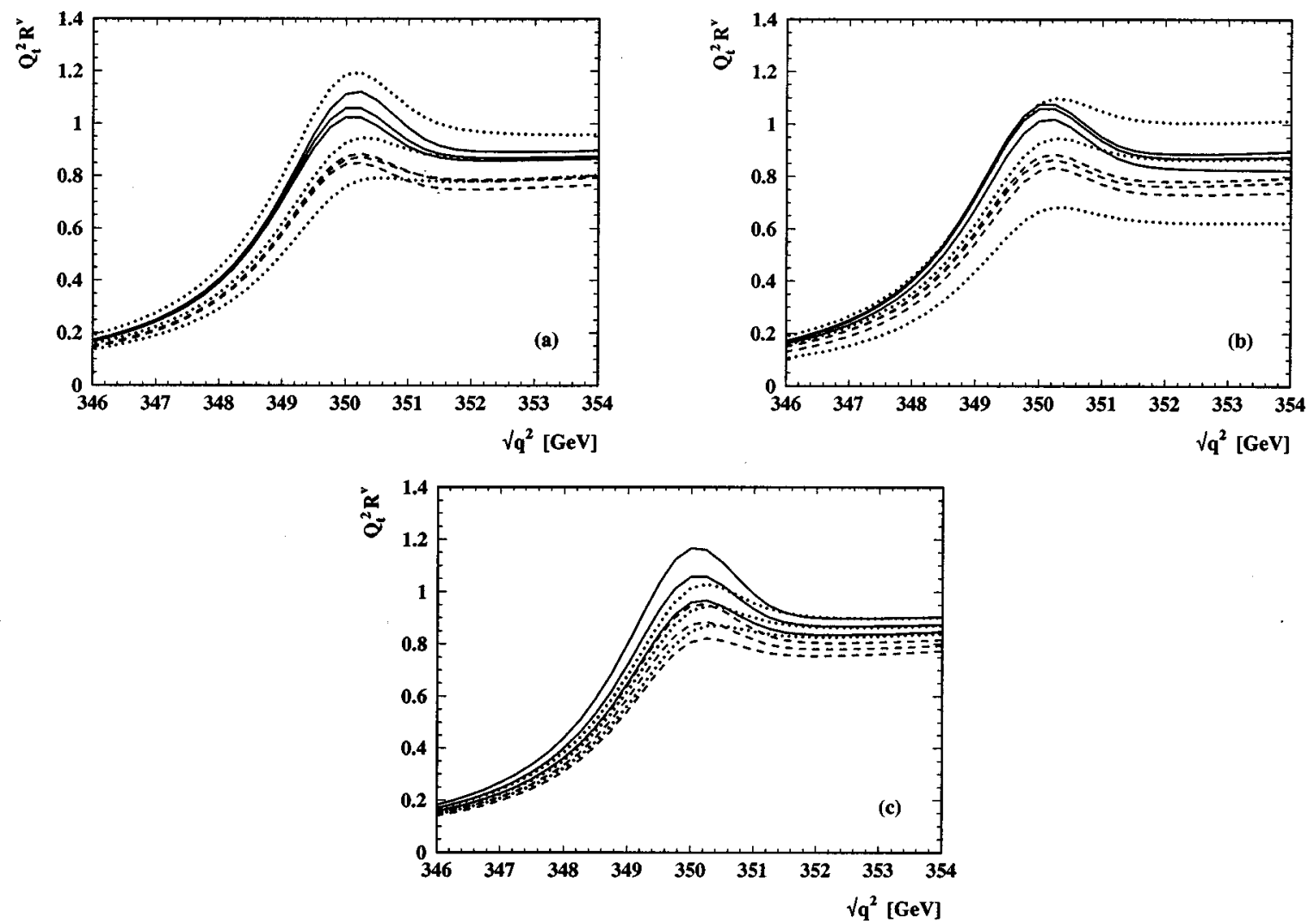

FIG. 7. The total vector current induced cross section $Q_{t}^{2} R^{\vee}$ for center-of-mass energies $346 \mathrm{GeV}<\sqrt{q^{2}}<354 \mathrm{GeV}$ in the $1 S$ mass scheme. The dependence on the renormalization scale $\mu(\mathrm{a})$, on the cutoff $\Lambda(\mathrm{b})$, and on $\alpha_{s}\left(M_{Z}\right)$ (c) is displayed. More details and the choice of parameters are given in the text.

It has been shown in Refs. $[53,50]$ that the RHS of Eq. (82) is equivalent to the dominant infrared contributions of the difference between the $\overline{\mathrm{MS}}$ and pole mass. Therefore the relation between $M_{1 S}$ and the $\overline{\mathrm{MS}}$ mass $\bar{m}_{t}$ only contains subleading infrared contributions, which are suppressed by at least one power of $1 / M_{t}$. In other words the ambiguity in the relation between $M_{1 S}$ and $\bar{m}_{t}$ is parametrically of order $\Lambda_{\mathrm{QCD}}^{2} / M_{t}$. This proves that $M_{1 S}$ is a short-distance mass. We also see from Eq. (82) that, if the pole mass is expressed in terms of the $1 S$ mass and if the resulting mass difference $2\left(M_{t}-M_{1 S}\right)$ is absorbed into the potential, the rest mass and the potential energy term contained in the total static energy are individually free of ambiguities of order $\Lambda_{\mathrm{QCD}}$. The RHS of Eq. (82) just subtracts the low momentum (i.e., dominant infrared sensitive) contribution from the Coulomb potential $V_{c}(\boldsymbol{x})$.

We note that in order to implement the $1 S$ mass definition into our numerical codes, which solve the integral equations (35) and (36), we have to invert relation (78). It has been shown in Refs. $[60,61]$ that a consistent way to achieve this task is to carry out the inversion with respect to the auxiliary parameter $\epsilon$. For the reason that this modified perturbative expansion has been applied for the first time to express inclusive $B$ decays in terms of the $\Upsilon(1 S)$ mass, it has been called the "Upsilon expansion"' $[60,61]$. If the $1 S$ mass is expressed in terms of the $\overline{\mathrm{MS}}$ mass, which is related to the pole mass by a series of the form $\bar{m}_{t}-M_{t}=M_{t} \Sigma_{n=1}^{\infty} a_{n} \alpha_{s}^{n}$, one has to consider a term $\propto \alpha_{s}^{n}$ in this relation of order $\epsilon^{n}$ in the Upsilon expansion. In other words, if one relates the $1 S$ mass to a mass which is different from the pole mass, one must combine terms of different order in $\alpha_{s}$. As an example, this means that in order to relate the $\mathrm{N}^{k} \mathrm{LO} 1 \mathrm{~S}$ mass to the $\overline{\mathrm{MS}}$ mass one needs to know its relation to the pole mass to $\mathcal{O}\left(\alpha_{s}^{k+1}\right)$. This is necessary because this is the only way in which the high order large perturbative corrections coming from infrared-sensitive terms are cancelled.

In Figs. 7 the total vector-current-induced cross section $Q_{t}^{2} R^{\vee}$ is displayed in the $1 S$ scheme for $346 \mathrm{GeV}<\sqrt{q^{2}}$ $<354 \mathrm{GeV}$ at LO (dotted lines), NLO (dashed lines), and NNLO (solid lines). In all figures shown in this section the top-quark width is chosen as $\Gamma_{t}=1.43 \mathrm{GeV}$ and the topquark $1 S$ mass as $M_{1 S}=175 \mathrm{GeV}$. Figure 7(a) displays the dependence on the renormalization scale for $\mu=15,30$, and $60 \mathrm{GeV}$ for $\alpha_{s}\left(M_{Z}\right)=0.118$ and $\Lambda=175 \mathrm{GeV}$. At LO and NNLO the choices $\mu=15,30$, and $60 \mathrm{GeV}$ correspond to the upper, middle, and lower curves. At NLO the choices $\mu=15$, 30 , and $60 \mathrm{GeV}$ correspond to the lower, middle, and upper curves for center-of-mass energies below the peak position. In Fig. 7(b) the dependence of $Q_{t}^{2} R^{v}$ on the choice of the cutoff $\Lambda$ is shown for $\alpha_{s}\left(M_{Z}\right)=0.118, \mu=30 \mathrm{GeV}$, and $\Lambda=90$ (lower curves), 175 (middle curves), and $350 \mathrm{GeV}$ (upper curves). Figure 7(c) displays the dependence of $Q_{t}^{2} R^{v}$ on the choice of $\alpha_{s}\left(M_{Z}\right)$ for $\alpha_{s}\left(M_{z}\right)=0.113$ (lower curves), 0.118 (middle curves), and 0.123 (upper curves) and $\Lambda=175$ $\mathrm{GeV}, \mu=30 \mathrm{GeV}$. Comparing the result with the curves dis- 

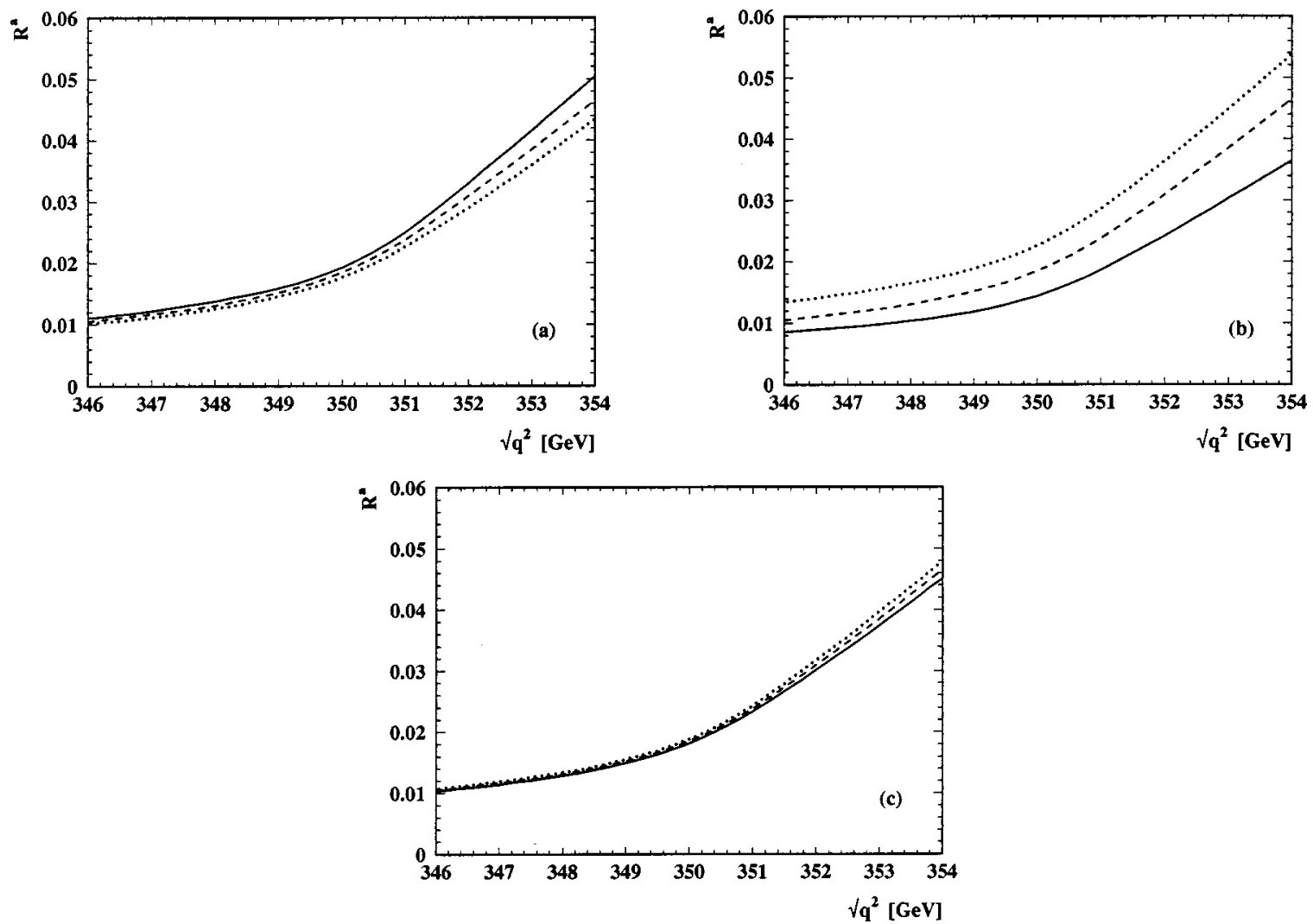

FIG. 8. The total axial-vector-current-induced cross section $R^{a}$ for center-of-mass energies $346 \mathrm{GeV}<\sqrt{q^{2}}<354 \mathrm{GeV}$ in the $1 S$ mass scheme. The dependence on the renormalization scale $\mu(\mathrm{a})$, on the cutoff $\Lambda(\mathrm{b})$, and on $\alpha_{s}\left(M_{Z}\right)$ (c) is displayed. More details and the choice of parameters are given in the text.

played in Figs. 3, the improvement of the stability of the peak position is evident. The strong dependence on the renormalization scale and the strong correlation with $\alpha_{s}\left(M_{Z}\right)$ have vanished. However, we also observe that the large corrections in the normalization of the curves are essentially not affected at all.

In Figs. 8(a)-8(c) the total axial-vector-current-induced cross section $R^{a}$ is displayed in the $1 S$ mass scheme for the same input parameters as in Fig. 7. Figure 8(a) shows the dependence on the renormalization scale for $\mu=15$ (solid line), 30 (dashed line), and $60 \mathrm{GeV}$ (dotted line), respectively. Figure 8(b) exhibits the dependence of the cutoff for $\Lambda=90$ (solid line), 175 (dashed line), and $350 \mathrm{GeV}$ (dotted line). Figure 4(c) shows $R^{a}$ for $\alpha_{s}\left(M_{z}\right)=0.113$ (solid line), 0.118 (dashed line), and 0.123 (dotted line). Compared to the plots in the pole mass scheme, we find a slightly smaller variation in the normalization with respect to the renormalization scale and the choice of $\alpha_{s}\left(M_{Z}\right)$. Clearly the effects of using the $1 S$ scheme instead of the pole one are much smaller in the axial-vector case because no peak is visible there.

In Figs. 9(a)-9(c) the LO (dotted lines), NLO (dashed lines), and NNLO (solid lines) top-quark-top-antiquark vector-current-induced three-momentum distribution $Q_{t}^{2} d R^{\mathrm{v}} / d\left|\boldsymbol{k}_{t}\right|$ is shown for $0<\left|\boldsymbol{k}_{t}\right|<60 \mathrm{GeV}$ in the $1 S$ mass scheme for center-of-mass energies exactly on top of the visible peak, $\sqrt{q^{2}}=M_{\text {peak }}$ and for $\sqrt{q^{2}}=M_{\text {peak }}+5 \mathrm{GeV}$. The input parameters have been chosen as in Figs. 7. Figure 9(a) shows the distributions for $\mu=15$ and $60 \mathrm{GeV}$. At LO and NNLO $\mu=15 \mathrm{GeV}$ corresponds to the upper curves and $\mu=60 \mathrm{GeV}$ to the lower curves for center-of-mass energies below the peak. At NLO $\mu=60 \mathrm{GeV}$ corresponds to the higher peak and $\mu=15 \mathrm{GeV}$ to the lower. Figure 9(b) displays the dependence of the distributions on the cutoff for $\Lambda=90$ (lower curves) and $350 \mathrm{GeV}$ (upper curves), and Fig. 9(c) exhibits the dependence of the distributions on the strong coupling for $\alpha_{s}\left(M_{Z}\right)=0.113$ and 0.123 . Below the peak the larger value of $\alpha_{s}\left(M_{Z}\right)$ always corresponds to the upper curve.

In Figs. 10(a)-10(c) the top-quark-top-antiquark axialvector-current-induced three-momentum distribution $d R^{a} / d\left|\boldsymbol{k}_{t}\right|$ is shown in the $1 S$ scheme for $0<\left|\boldsymbol{k}_{t}\right|<60 \mathrm{GeV}$ and both for center-of-mass energies exactly on top of the visible peak, $\sqrt{q^{2}}=M_{\text {peak }}$, and for $\sqrt{q^{2}}=M_{\text {peak }}+5 \mathrm{GeV}$. The input parameters have been chosen as before. Figure 10(a) shows the distribution for $\mu=15$ (solid curves), 30 (dashed curves), and $60 \mathrm{GeV}$ (dotted curves). Figure 10(b) displays the dependence of the distribution on the cutoff for $\Lambda=90$ (solid curves), 175 (dashed curves), and $350 \mathrm{GeV}$ (dotted curves), and Fig. 10(c) exhibits the dependence of the distribution on the strong coupling for $\alpha_{s}\left(M_{Z}\right)=0.113$ (solid curves), 0.118 (dashed curves), and 0.123 (dotted curves). The curves shown in Figs. 10 are somewhat higher than in Figs. 6, because the choice of $175 \mathrm{GeV}$ for the top-quark 

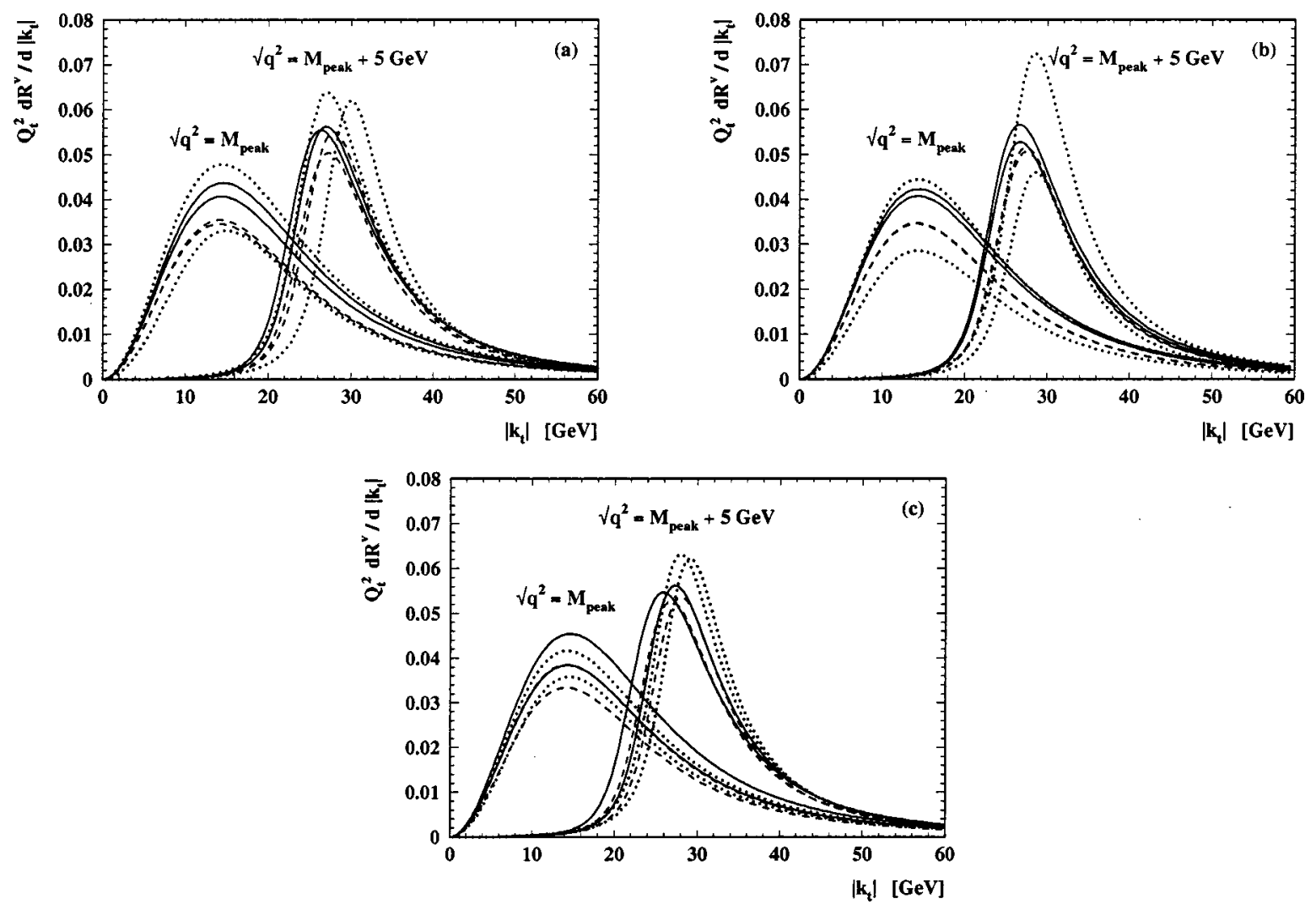

FIG. 9. The three-momentum distribution of the vector-current-induced cross section $Q_{t}^{2} R^{\vee}$ for center-of-mass energies $\sqrt{q^{2}}=M_{\text {peak }}$ and $M_{\text {peak }}+5 \mathrm{GeV}$ in the $1 S$ mass scheme. The dependence on the renormalization scale $\mu$ (a), on the cutoff $\Lambda$ (b), and on $\alpha_{s}\left(M_{Z}\right)$ (c) is displayed. More details and the choice of parameters are given in the text.

mass corresponds to a higher value for $M_{\text {peak }}$ in the $1 S$ scheme. From Figs. 9 and 10 it is evident that the $1 S$ scheme does not essentially affect at all the three-momentum distributions. Compared to the results in the pole mass scheme the variations of the peak position remain unchanged. This can be understood from the fact that a mass redefinition corresponds to a shift in the center-of-mass energy, but leaves the definition of the off-shell top quark three-momentum unchanged.

In Table II we have displayed the LO, NLO, and NNLO corrections to the peak position with respect to $2 M_{1 S}$ :

$$
\begin{aligned}
M_{\text {peak }} & =2 M_{1 S}+\delta M_{\text {peak }, 1 S}^{\mathrm{LO}}+\delta M_{\text {peak }, 1 S}^{\mathrm{NLO}}+\delta M_{\text {peak }, 1 S}^{\mathrm{NNL}} \\
& =2 M_{1 S}+\Delta M_{\text {peak }, 1 S},
\end{aligned}
$$

in the $1 S$ mass scheme for $M_{1 S}=175 \mathrm{GeV}, \Gamma_{t}=1.43 \mathrm{GeV}$, $\alpha_{s}\left(M_{Z}\right)=0.113,0.118$, and 0.123 , and $\mu=15,30$, and 60 $\mathrm{GeV}$ for various choices of the renormalization scale $\mu$ and the strong coupling $\alpha_{s}\left(M_{Z}\right)$. Taking the size of the NLO and NNLO corrections as a measure for the present theoretical uncertainty in the peak position, and assuming that the latter can be used to estimate the theoretical uncertainty in the determination of $M_{1 S}$, we find that this uncertainty is approximately $200 \mathrm{MeV}$. If the effects of beamstrahlung and initial state radiation at a future $e^{+} e^{-}$or muon pair collider do not spoil a precise determination of the $1 S$ mass with an uncertainty of $200 \mathrm{MeV}$ one has to ask the question how $M_{1 S}$ is related to the top-quark mass parameters usually used for calculations of physical observables that are not related to the threshold regime. In principle, one could use $M_{1 S}$ as a new top-quark mass parameter in its own right. This would, of course, require that all formulas be expressed in terms of $M_{1 S}$, using the Upsilon expansion discussed after Eq. (82). A more economical way is to relate the $1 S$ mass to the $\overline{\mathrm{MS}}$ top-quark mass, which is a common mass parameter for perturbative calculations involving heavy quarks and which, in a number of cases, even leads to improved convergence properties of the perturbative series. ${ }^{15}$ Because the $1 S$ mass is a short-distance mass, its perturbative relation to the $\overline{\mathrm{MS}}$ mass is much better behaved at large orders than the corresponding relation of the pole mass. The relation between $M_{1 S}$ and $\bar{m}_{t}\left(\bar{m}_{t}\right)$ can be derived from Eq. (78) and the relation between pole mass and $\bar{m}_{t}\left(\bar{m}_{t}\right)$ using the Upsilon expansion discussed above. We emphasize that the three-loop relation between the pole and the $\overline{\mathrm{MS}}$ mass is needed to relate $M_{1 S}$ and $\bar{m}_{t}\left(\bar{m}_{t}\right)$ at NNLO accuracy. Assuming that those threeloop corrections can be approximated by the known corrections in the large- $\beta_{0}$ limit [55], we find the following numerical value for $\bar{m}_{t}\left(\bar{m}_{t}\right)$ for $M_{1 S}=175 \pm 0.2 \mathrm{GeV}$ and $\alpha_{s}\left(M_{Z}\right)=0.118 \pm x 0.001$,

\footnotetext{
${ }^{15}$ Prominent cases are the top quark QCD corrections to the $\rho$ parameter [62] and the massive quark pair production cross section at large energies [63].
} 

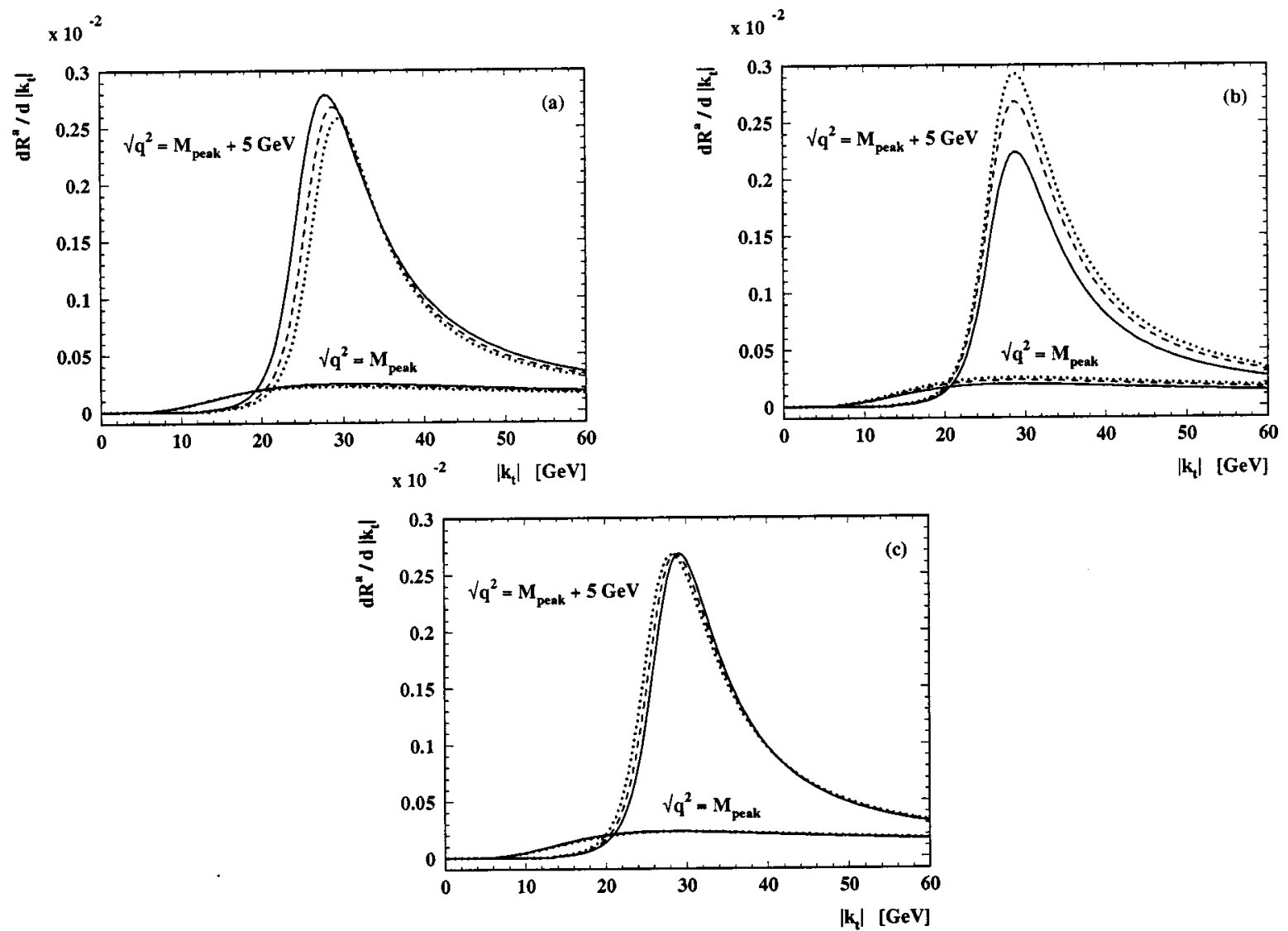

FIG. 10. The three-momentum distribution of the axial-vector-current-induced cross section $R^{a}$ for center-of-mass energies $\sqrt{q^{2}}$ $=M_{\text {peak }}$ and $M_{\text {peak }}+5 \mathrm{GeV}$ in the $1 S$ mass scheme. The dependence on the renormalization scale $\mu$ (a), on the cutoff $\Lambda$ (b), and on $\alpha_{s}\left(M_{Z}\right)$ (c) is displayed. More details and the choice of parameters are given in the text.

$$
\begin{aligned}
\bar{m}_{t}\left(\bar{m}_{t}\right)= & {\left[175-7.58 \epsilon(\mathrm{LO})-0.96 \epsilon^{2}(\mathrm{NLO})\right.} \\
& -0.23 \epsilon^{3}(\mathrm{NNLO}) \pm 0.2\left(\delta M_{1 S}\right) \\
& \left. \pm x 0.07\left(\delta \alpha_{s}\right)\right] \mathrm{GeV} .
\end{aligned}
$$

For the numbers given in Eq. (84) we have assumed an un-

TABLE II. LO, NLO, and NNLO contributions to the peak position of the total vector-current-induced cross section $R^{\mathrm{v}}$ in $\mathrm{GeV}$ in the $1 S$ mass scheme for $M_{1 S}=175 \mathrm{GeV}, \Gamma_{t}=1.43 \mathrm{GeV}$, $\alpha_{s}\left(M_{Z}\right)=0.113,0.118$, and 0.123 , and $\mu=15,30$, and $60 \mathrm{GeV}$, respectively. For the strong coupling two-loop running has been employed. The results are insensitive to the choice of the cutoff scale $\Lambda \sim 175 \mathrm{GeV}$.

\begin{tabular}{cccccc}
\hline \hline$\mu[\mathrm{GeV}]$ & $\alpha_{s}\left(M_{Z}\right)$ & $\delta M_{\text {peak,1S }}^{\mathrm{LO}}$ & $\delta M_{\text {peak,1S }}^{\mathrm{NLO}}$ & $\delta M_{\text {peak,1S }}^{\mathrm{NNLO}}$ & $\delta M_{\text {peak,1S }}$ \\
\hline 15 & 0.113 & 0.21 & 0.03 & -0.03 & 0.20 \\
30 & & 0.38 & -0.11 & -0.09 & 0.17 \\
60 & & 0.78 & -0.50 & -0.11 & 0.17 \\
15 & 0.118 & 0.16 & 0.02 & -0.00 & 0.17 \\
30 & & 0.30 & -0.09 & -0.08 & 0.12 \\
60 & & 0.54 & -0.32 & -0.10 & 0.11 \\
15 & 0.123 & 0.12 & -0.00 & 0.04 & 0.16 \\
30 & & 0.23 & -0.07 & -0.08 & 0.08 \\
60 & & 0.42 & -0.26 & -0.10 & 0.07 \\
\hline \hline
\end{tabular}

certainty in the value of the strong coupling at $M_{Z}$ of $x 0.001$ in order to demonstrate the importance of $\alpha_{s}$ for the determination of $\bar{m}_{t}\left(\bar{m}_{t}\right)$. This uncertainty is independent of the order to which the relation between the pole and the $\overline{\mathrm{MS}}$ mass is known because it comes from the LO term. We note that this fact shows that the strong correlation of the peak position to the strong coupling, which was visible in the pole mass scheme, is not necessarily eliminated by adopting the $1 S$ scheme. This correlation might come back whenever the $1 S$ mass is related to another short-distance mass or is used as a parameter in other quantities. However, the use of the $1 S$ mass has the advantage to free the process of the mass extraction from the total cross section close to threshold also from strong dependences on other parameters such as the renormalization scale or the order of approximation used. Therefore systematic uncertainties are expected to be smaller if the $1 S$ scheme is used for the threshold calculations. Equation (84) shows that the knowledge of the three-loop corrections in the relation of pole and $\overline{\mathrm{MS}}$ mass and a small uncertainty in $\alpha_{s}\left(M_{Z}\right)$ are crucial for a determination of $\bar{m}_{t}\left(\bar{m}_{t}\right)$ with uncertainties comparable to $\delta M_{1 S}$.

In recent literature there have been two other proposals for alternative short-distance mass definitions, which can also be used for a measurement of the top-quark mass from the total cross section. In Refs. $[64,65]$ the "low scale running mass" was proposed to subtract the infrared behavior 
from the heavy quark self-energy. The "low scale running mass" was devised in order to improve the convergence of the perturbative series describing the contributions leading in $1 / M_{b}$ in inclusive $B$-meson decays. Because of the universality of the dominant infrared sensitive contribution, the low scale running mass can also serve as a top-quark mass definition, which leads to an improved stability of the peak position in the total cross section. The low scale running mass depends on the cutoff $\mu_{\mathrm{LS}}$, which limits the momenta that are subtracted from the self-energy. At order $\alpha_{s}$ (i.e., at LO) its relation to the pole mass reads $[64,65]$

$$
m_{t}^{\mathrm{LS}}\left(\mu_{\mathrm{LS}}\right)-M_{t}=-\frac{16}{9} \frac{\alpha_{s}}{\pi} \mu_{\mathrm{LS}}\left[1+\mathcal{O}\left(\alpha_{s}\right)+\mathcal{O}\left(\frac{\mu_{\mathrm{LS}}}{M_{t}}\right)\right] .
$$

By adjusting the scale $\mu_{\mathrm{LS}}$ in such a way that the RHS of Eq. (85) is comparable in size to the RHS of Eq. (78) the position of the peak in the total cross section can be stabilized. In Ref. [53] the "potential-subtracted" mass was proposed. It subtracts the dominant infrared-sensitive contribution in the Schrödinger equation (19), which is contained in the static potential $V_{c}$. The subtraction is in fact equal to the RHS of Eq. (82). Like the low scale running mass, the potentialsubtracted mass depends on a cutoff $\mu_{\mathrm{PS}}$. At order $\alpha_{s}$ (LO) the relation to the pole mass reads [53]

$$
m_{t}^{\mathrm{PS}}\left(\mu_{\mathrm{PS}}\right)-M_{t}=-\frac{4}{3} \frac{\alpha_{s}}{\pi} \mu_{\mathrm{PS}}\left[1+\mathcal{O}\left(\alpha_{s}\right)\right] .
$$

As for the low scale running mass, the scale $\mu_{\mathrm{PS}}$ can be adjusted in such a way that the RHS of Eq. (86) is comparable in size to the RHS of Eq. (78). To achieve this, $\mu_{\mathrm{PS}}$ has to be chosen of the order of the inverse Bohr radius $\sim M_{t} \alpha_{s}$, which is much larger than the scale $\mu_{f} \ll M_{t} \alpha_{s}$ introduced in Eq. (82). For $\mu_{\mathrm{PS}}=\frac{4}{3} \mu_{\mathrm{LS}}$ the low scale running and the potential-subtracted mass lead to approximately equivalent results. However, the stabilization of the peak position can be expected to be slightly worse than for the $1 S$ mass if $\mu_{\mathrm{PS}}$ or $\frac{4}{3} \mu_{\mathrm{LS}}$ are not fine-tuned. In addition, the results that could finally be obtained for the $\overline{\mathrm{MS}}$ top mass can depend on the value that is chosen for the cutoff scale $\mu_{\mathrm{LS}}$ and $\mu_{\mathrm{PS}}$.

\section{B. Normalization of the total cross section}

In the previous subsection we have demonstrated that a proper redefinition of the top-quark mass leads to a considerable improvement in the stability of the peak position in the vector-current-induced total cross section $R^{v}$. However, there have been only marginal changes in the size of the NNLO corrections to the overall normalization of the line shape. Compared to the NLO normalization of the total vector-current-induced cross section, the NNLO corrections are between 15 and $25 \%$, which is rather large if one recalls that the NNLO corrections are parametrically of order $v^{2}$ $\sim \alpha_{s}^{2}$. In this section we try to find some answers to the question, whether the large NNLO corrections to the normalization of the total vector-current-induced cross section have to be interpreted as a sign that the nonrelativistic expansion for the top-quark-top-antiquark cross section close to threshold breaks down. Clearly, this question can only be answered reliably after the complete $\mathrm{N}^{3} \mathrm{LO}$ corrections have been determined, which are, unfortunately, beyond the capabilities of present technology. We therefore analyze the NNLO corrections to the normalization of the total cross section with respect to their sensitivity to infrared momenta and carry out a comparison to the one- and two-loop cross section for energies far above the top-quark-top-antiquark threshold, where conventional perturbation theory in $\alpha_{s}$ is believed to be reliable. We provide arguments that the large NNLO corrections to the normalization are genuine $\mathcal{O}\left(\mathrm{v}^{2}, \alpha_{s}^{2}\right)$ relativistic corrections, which cannot be removed by changing the definition of $\alpha_{s}$ or the top-quark mass, and that their size does not necessarily indicate a breakdown of the nonrelativistic expansion used in this work.

As far as a redefinition of the top quark mass is concerned, it is quite obvious that it cannot significantly affect the normalization of the total cross section because the dominant effect in a mass shift is an energy shift of the entire line shape. Nevertheless, it is quite interesting that the normalization is at all insensitive to the dominant infrared-sensitive terms in the Schrödinger equation (19),${ }^{16}$ which, in the pole mass scheme, would cause the corrections to the peak position to grow factorially at large orders of perturbation theory. To show this let us recall that the total vector-currentinduced cross section is proportional to the absorptive part of the Green function, with both arguments evaluated at the origin in configuration space representation:

$$
R^{\vee} \sim \operatorname{Im} \oiint_{n} \frac{\left|\Phi_{n}(0)\right|^{2}}{E_{n}-E-i \Gamma_{t}}
$$

where the sum extends over discrete and continuum states with $S$ wave quantum numbers. Thus, for fixed energy the normalization only depends on the wave function. Repeating the steps following Eq. (80) we find that the correction to the wave function coming from the dominant infrared-sensitive terms in the Schrödinger equation reads

$$
\begin{aligned}
{\left[\delta \Phi_{n}(0)\right]^{\mathrm{IR}}=} & {\left[\int \frac{d^{3} \mathbf{p}}{(2 \pi)^{3}} \int \frac{d^{3} \mathbf{q}}{(2 \pi)^{3}}\right.} \\
& \left.\times \sum_{m \neq n} \frac{\Phi_{m}(0) \tilde{\Phi}_{m}^{*}(\mathbf{p})}{E_{m}-E-i \Gamma_{t}} \delta \mathcal{H}(\mathbf{p}, \mathbf{q}) \tilde{\Phi}_{n}(\mathbf{q})\right]^{\mathrm{IR}} \\
& \int \frac{d^{3} \mathbf{p}}{(2 \pi)^{3}} \sum^{|\mathbf{q}|<\mu_{f}} \frac{d^{3} \mathbf{q}}{(2 \pi)^{3}} \oiint_{m \neq n} \frac{\Phi_{m}(0) \tilde{\Phi}_{m}^{*}(\mathbf{p})}{E_{m}-E-i \Gamma_{t}} \\
& \times \widetilde{\Phi}_{n}(\mathbf{p}) \delta \tilde{V}_{c}(\mathbf{q})=0,
\end{aligned}
$$

\footnotetext{
${ }^{16}$ In our case the two issues are in fact connected to each other. But it is important to conceptually separate the issue of a simple energy shift from the more fundamental question of infrared sensitivity.
} 


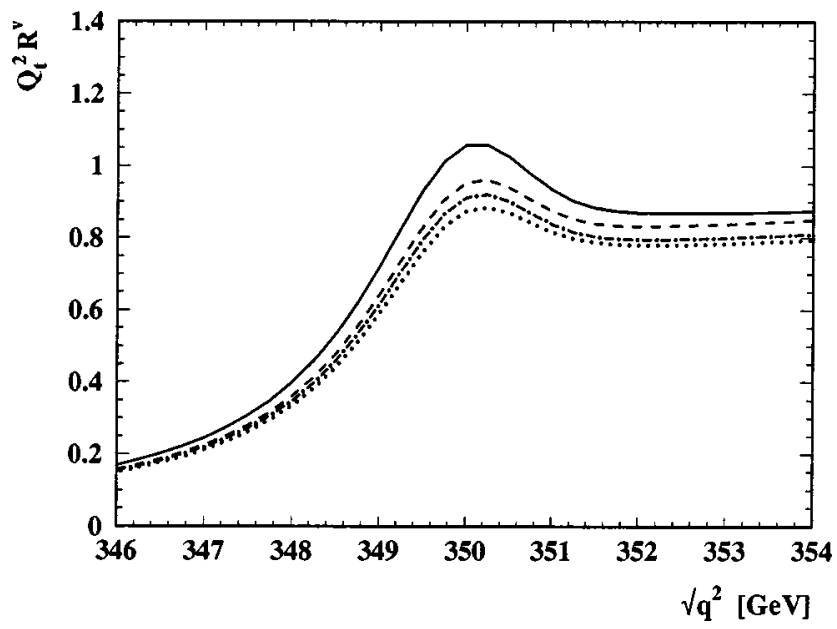

FIG. 11. The total vector-current-induced cross section $Q_{t}^{2} R^{v}$ close to threshold for $M_{1 S}=\Lambda=175 \mathrm{GeV}, \mu=30 \mathrm{GeV}$, $\alpha_{s}\left(M_{Z}\right)=0.118$, and $\Gamma_{t}=1.43 \mathrm{GeV}$ at NLO (dotted curve) and NNLO (solid curve). The dash-dotted curve is NLO including also the NNLO corrections to the Coulomb potential $V_{c}$, and the dashed line contains, in addition, all Abelian NNLO corrections. The differences between the curves indicates the size of individual NNLO relativistic corrections.

i.e., it vanishes because of the orthogonality of the wave functions. Therefore the large corrections in the normalization of the total cross section are not related to an infrared sensitivity of the corrections, in particular at large orders. To demonstrate that this is also the case for the NNLO corrections calculated in this work, we have displayed in Fig. 11 the total vector-current-induced cross section $Q_{t}^{2} R^{\vee}$ close to threshold for $M_{1 S}=\Lambda=175 \mathrm{GeV}, \quad \mu=30 \mathrm{GeV}$, $\alpha_{s}\left(M_{Z}\right)=0.118$, and $\Gamma_{t}=1.43 \mathrm{GeV}$ successively including various NNLO corrections. The dotted line represents the NLO cross section and the solid line the NNLO one. The dash-dotted line is the NLO cross section including also the NNLO corrections to the Coulomb potential $V_{c}$; the dashed line contains, in addition, all Abelian NNLO corrections, i.e., those that do not involve the $\mathrm{SU}(3)$ group theoretical factor $C_{A}$. The separation of the NNLO corrections into those coming from the Coulomb potential and from Abelian and non-Abelian relativistic corrections is gauge invariant. The difference between the dashed and the solid curve represents the corrections of the non-Abelian NNLO effects originating from the potential $V_{\mathrm{NA}}$ and those $\mathcal{O}\left(\alpha_{s}^{2}\right)$ contributions to the short-distance coefficient $C^{\vee}$ that are proportional to $C_{A}$. From the rather small difference between the dotted and the dash-dotted curves (2-4\%) we see that the large NNLO corrections to the normalization are not related to the corrections in the Coulomb potential. Because a redefinition of the strong coupling would mainly affect the size of the higherorder corrections in the Coulomb potential, we can conclude that using a different scheme for the strong coupling (such as the $V$ scheme $[66,67]$ ) will not significantly affect the size of the NNLO corrections. The curves plotted in Fig. 11 demonstrate that the $\mathcal{O}(20 \%)$ NNLO correction to the normalization is a sum of corrections, each of which positive and individually either smaller than or approximately equal to $\mathcal{O}(10 \%)$.
Although this observation, of course, cannot be taken as a proof that the still unknown $\mathrm{N}^{3} \mathrm{LO}$ corrections are smaller than the NNLO ones, it indicates that the size of the latter does not necessarily have to be taken as an argument for the non-relativistic expansion to break down for the normalization of the total cross section.

An interesting insight into the question of how to interpret the large normalization corrections can also be obtained by comparing the total cross section line shape, which we have calculated in the threshold regime, with earlier calculations of the total cross section for higher energies, where a resummation of Coulomb singular terms is not yet necessary and perturbation theory in $\alpha_{s}$ is believed to be reliable [68]. We would like to note that it is the large mass of the top quark that allows us to draw conclusions from a comparison of the threshold cross section with the one calculated for higher energies. To illustrate this we recall that our calculation of the threshold cross section is valid if the hierarchy $\alpha_{s}, \mathrm{~V}$ $\ll 1$ is satisfied, where the scale of the strong coupling is of the order of the inverse Bohr radius, the kinetic energy, or the top-quark width. This means that the threshold cross section represents a simultaneous expansion in $\alpha_{s}$ and $\mathrm{v}$, where powers of $\left(\alpha_{s} / \mathrm{V}\right)$ are resummed to all orders in $\alpha_{s}$. The high energy cross section, on the other hand, is valid if $\alpha_{s}$ $\ll \mathrm{v}, 1$, where the scale in the strong coupling is of order the top-quark-top-antiquark relative momentum or the centerof-mass energy. Thus a comparison of the threshold results with the high energy perturbative ones is only sensible if there exists a kinematic regime where both hierarchies are satisfied at the same time, i.e., if $\alpha_{s} \ll \mathrm{V} \ll 1$. In this regime the effects of the resummation of powers of $\left(\alpha_{s} / \mathrm{v}\right)$ not contained in the high energy cross section should be small as well as the effects of velocity corrections beyond NNLO, which are not contained in the threshold cross section. Obviously this relation is difficult or impossible to satisfy for bottom or charm quarks, but it is possible for the top-quark case. For $\alpha_{s}\left(M_{t} \alpha_{s}\right) \sim 0.13$ we can argue that a meaningful comparison between threshold and high energy cross section should be possible for $\mathrm{V} \approx 0.3-0.4$, which corresponds to $\sqrt{s} \approx 365 \mathrm{GeV}$. In Fig. 12 we have plotted the threshold and the high energy cross sections at NLO/NNLO and $\mathcal{O}\left(\alpha_{s}\right) / \mathcal{O}\left(\alpha_{s}^{2}\right)$, respectively, for the renormalization scales $\mu=25 \mathrm{GeV}$ (dotted lines), $2\left(p_{0}^{4}+M_{t}^{2} \Gamma_{t}^{2}\right)^{1 / 4}$ (solid lines), 175 $\mathrm{GeV}$ (dashed lines), and $\sqrt{q^{2}}$ (dash-dotted lines) for $M_{t}=\Lambda=175 \mathrm{GeV}, \alpha_{s}\left(M_{Z}\right)=0.118$, and $\Gamma_{t}=1.43$. The lower bunch of threshold curves (characterized by the peak at around $\sqrt{q^{2}}=348 \mathrm{GeV}$ ) is NLO and the upper bunch NNLO. Likewise, the lower bunch of high energy curves is $\mathcal{O}\left(\alpha_{s}\right)$ and the upper bunch $\mathcal{O}\left(\alpha_{s}^{2}\right)$. We note that we have not plotted the threshold curves for $\mu=\sqrt{q^{2}}$ and the high energy curves not for $\mu=25 \mathrm{GeV}$, which seems to be a rather unnatural choice for each. The formulas for the $\mathcal{O}\left(\alpha_{s}^{2}\right)$ high energy cross section have been taken from Ref. [68]. For convenience we have plotted the curves in Fig. 12 in the pole mass scheme. Because the choice of the mass definition does not alter the behavior of the cross section normalization for energies above the peak position, this choice does not affect the conclusions drawn below. For the threshold (high en- 


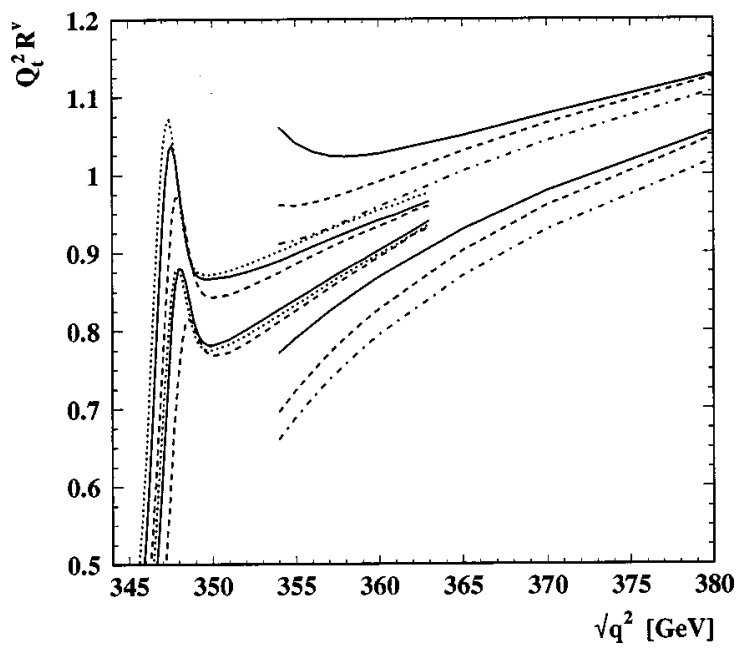

FIG. 12. The vector-current-induced total cross section in the nonrelativistic expansion at NLO (lower bunch of threshold curves), NNLO (upper bunch), and in conventional perturbation theory at $\mathcal{O}\left(\alpha_{s}\right)$ (lower bunch of high energy curves) and $\mathcal{O}\left(\alpha_{s}^{2}\right)$ (upper bunch). The pole mass scheme has been used. The curves have been plotted for $\alpha_{s}\left(M_{z}\right)=0.118, \Gamma_{t}=1.43 \mathrm{GeV}, M_{t}=\Lambda=175$ $\mathrm{GeV}$, and $\mu=25 \mathrm{GeV}$ (dotted lines), $2\left(p_{0}^{4}+M_{t}^{2} \Gamma_{t}^{2}\right)^{1 / 4}$ (solid lines), $175 \mathrm{GeV}$ (dashed lines), and $\sqrt{q^{2}}$ (dash-dotted lines). The formulas for the $\mathcal{O}\left(\alpha_{s}^{2}\right)$ high energy cross section have been taken from Ref. [68].

ergy) cross section, we observe that the $\mathrm{NNLO}\left[\mathcal{O}\left(\alpha_{s}^{2}\right)\right]$ corrections decrease for energies further away from the threshold region. However, the $\mathcal{O}\left(\alpha_{s}^{2}\right)$ corrections to the high energy cross sections are much larger than the NNLO corrections to the threshold cross section at the same center-ofmass energy. For $\sqrt{s}=360-370 \mathrm{GeV}$ the $\mathcal{O}\left(\alpha_{s}^{2}\right)$ corrections to the high energy cross section are, for equal choices of renormalization scales, between 10 and $20 \%$ compared to only around $5 \%$ for the NNLO corrections to the threshold cross section. We also see a much weaker renormalization scale dependence of the threshold cross sections. The curves show that the resummation of Coulomb singular terms contained in the threshold calculation leads to a considerable stabilization of the cross section determined in conventional perturbation theory in $\alpha_{s}$ for energies below $\sqrt{s}=365 \mathrm{GeV}$. If we believe that conventional perturbation theory is reliable down to energies around $\sqrt{s}=360 \mathrm{GeV}$, the results displayed in Fig. 12 indicate that the nonrelativistic expansion does certainly not break down. However, the curves of Fig. 12 also make it evident that the small renormalization scale dependence of the NLO threshold cross section does certainly not reflect the true size of the remaining theoretical uncertainties at NLO. We believe that $10 \%$ should be a fair estimate of the remaining theoretical uncertainties contained in the normalization of the NNLO total cross section close to threshold. As far as the top-quark mass determination at a future electron-positron linear or muon pair collider is concerned, this rather large normalization uncertainty might in fact lead to uncertainties in the determination of the $1 S$ mass that are larger than indicated in the previous subsection. This is due to the effects of beamstrahlung and initial state radia- tion that lead to a smearing of the effective center-of-mass energy of about 1-2 GeV [4]. Beamstrahlung and initial state radiation render the visible peak in the total cross section either smaller (at the muon pair collider) or completely invisible (at the linear collider), which makes it possible that the uncertainty in the normalization feeds into larger uncertainties in the determination of $M_{1 S}$. It is the task of realistic simulation studies to determine how large this effect is for the various collider and detector designs and to devise optimized strategies to minimize it. If the effects of beamstrahlung and initial state radiation on the top quark mass determination are small, the uncertainty in the normalization will mainly affect the measurement of the strong coupling [see Figs. 7(c) and 13(b)].

In Figs. 13 the total cross section $\sigma_{\text {tot }}^{\gamma, Z}\left(e^{+} e^{-} \rightarrow \gamma^{*}, Z^{*}\right.$ $\rightarrow \bar{t})$, Eq. (8), is plotted at NNLO in the $1 S$ scheme for $M_{1 S}=\Lambda=175 \mathrm{GeV}, \Gamma_{t}=1.43 \mathrm{GeV}$, and $\alpha=1 / 125.7$. Figure 13(a) shows the renormalization scale dependence for $\mu=15$ (solid line), 30 (dashed line), and $60 \mathrm{GeV}$ (dotted line), and $\alpha_{s}\left(M_{Z}\right)=0.118$. Figure 13(b) displays the dependence on the strong coupling for $\alpha_{s}\left(M_{Z}\right)=0.113$ (solid line), 0.118 (dashed line), and 0.123 (dotted line), and for $\mu=30 \mathrm{GeV}$.

\section{SUMMARY AND CONCLUSIONS}

Within the framework of the nonrelativistic effective field theories NRQCD and PNRQCD, we have calculated the vector-current-induced total cross section of top-quark-topantiquark pair production in electron-positron annihilation close to threshold at NNLO in the nonrelativistic expansion. The corresponding NNLO QCD relativistic corrections have also been determined for the vector-current-induced topquark three-momentum distribution. In addition, the axialvector-current-induced total cross section and the threemomentum distribution have been calculated to fully account for the Z-boson contributions in electron-positron annihilation. For the total cross section and the three-momentum distribution, the axial-vector-current-induced contributions are suppressed by $v^{2}$ with respect to the vector current contributions; they have therefore been determined in leading order in the nonrelativistic expansion. The size of the axialvector-current-induced contributions is smaller than the remaining theoretical uncertainties in the vector-currentinduced cross section (for unpolarized electrons and positrons). In contrast with previous literature on the same subject, we have implemented the top-quark width by including electroweak corrections into the (P)NRQCD matching conditions of the Lagrangian and the currents. This allows for a straightforward generalization of the Fadin-Khoze prescription " $E \rightarrow E+i \Gamma_{t}$ " to implement the top-quark width at NNLO in the nonrelativistic expansion, where $\Gamma_{t} / M_{t}$ is counted as order $\mathrm{v}^{2}$. We have shown that at NNLO this cannot be achieved by a simple shift of the center-of-mass energy to complex values. Our calculations have been carried out using numerical techniques to solve the corresponding integral equations within a cutoff regularization scheme and using analytic methods for the matching procedure. We have addressed the question of large NNLO corrections to the peak position and the normalization of the total vector- 

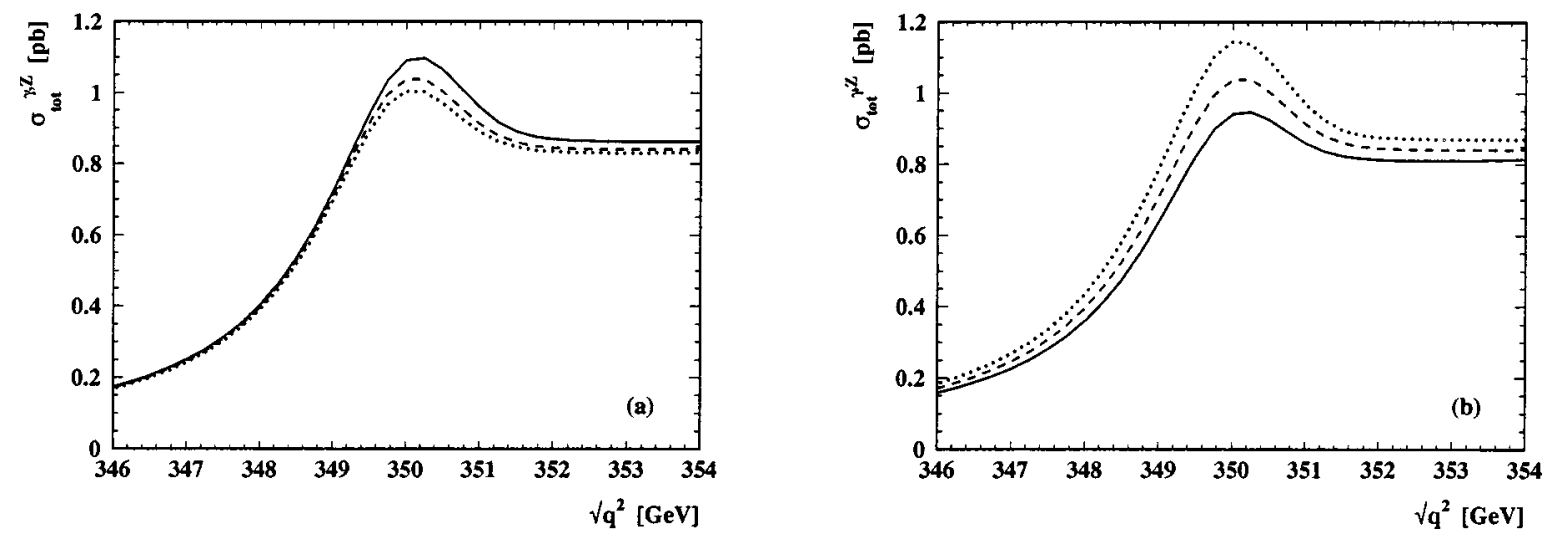

FIG. 13. The total cross section $\sigma_{\text {tot }}^{\gamma, Z}$, Eq. (8), is plotted in the $1 S$ scheme at NNLO for $M_{1 S}=\Lambda=175 \mathrm{GeV}, \Gamma_{t}=1.43 \mathrm{GeV}$, and $\alpha=1 / 125.7$. (a) shows the renormalization scale dependence for $\mu=15$ (solid line), 30 (dashed line), and $60 \mathrm{GeV}$ (dotted line), and $\alpha_{s}\left(M_{Z}\right)=0.118$; (b) shows the dependence on the strong coupling for $\alpha_{s}\left(M_{Z}\right)=0.113$ (solid line), 0.118 (dashed line), and 0.123 (dotted line), and $\mu=30 \mathrm{GeV}$.

current-induced cross section. The position of the peak, which is observable in the total vector-current-induced cross section, can be stabilized if the cross section is expressed in terms of the $1 S$ mass, instead of the pole mass. The $1 S$ mass $M_{1 S}$ is defined as half the mass of a fictitious ${ }^{3} S_{1}$ toponium ground state for a stable top quark. The $1 S$ mass is a shortdistance mass and, by construction, reduces to a large extent the dependence of the peak position on theoretical parameters such as the renormalization scale of the strong coupling. We have also shown that the large NNLO corrections to the normalization of the total cross section, of order $20 \%$, are genuine NNLO corrections, which cannot be removed by a redefinition of the top-quark mass or the strong coupling. The large size of the corrections to the normalization originates from the fact that NNLO relativistic corrections from several sources have the same sign. We believe that the remaining theoretical uncertainties in the normalization are of order $10 \%$. If the effects of beamstrahlung and initial state radiation at the $e^{+} e^{-}$linear collider do not lead to a significant cross feed of the uncertainties in the normalization into $M_{1 S}$, we expect that an uncertainty in the determination of $M_{1 S}$ of less than $200 \mathrm{MeV}$ will be possible at the linear collider with an integrated luminosity of $50-100 \mathrm{fb}^{-1}$. In order to determine the $\overline{\mathrm{MS}}$ top-quark mass from the $1 S$ mass with the same precision, the knowledge of the full three-loop relation between the pole and the $\overline{\mathrm{MS}}$ mass, and a small uncertainty in $\alpha_{s}\left(M_{Z}\right)$ are crucial.

After completion of this work, we received Refs. [69-71]. In Ref. [69] the total vector-current-induced cross section has been calculated analytically, using the $\overline{\mathrm{MS}}$ regularization scheme based on the Schrödinger equation (19). The NLO and NNLO corrections have been treated perturbatively, supplemented by a resummation of the energy denominators for the $n=1$ and $n=2$ states in the spectral representation of the Green function. The renormalization scale dependence of the cross section line shape is considerably larger in Ref. [69] than in our work. This might be a consequence of the perturbative treatment of the NLO and NNLO corrections. In addition, a next-to-leading logarithmic resummation of logarithms of the ratio $M_{t} / \mu$ in the short-distance coefficient
$C{ }_{\overline{\mathrm{MS}}}^{\mathrm{v}}$ has been carried out, taking the $\overline{\mathrm{MS}}$ cutoff scale $\mu$ of order $M_{t} \mathrm{v}$. The effect of this resummation is around $5 \%$ for the normalization of the total cross section. In our cutoff scheme, where the regularization scale is of order $M_{t}$, the corresponding logarithm is contained in the current correlators. In Ref. [70] the vector-current-induced cross total section and the three-momentum distribution have been calculated at NNLO, based on the simplified Schrödinger equation (70), which we have discussed critically at the end of Sec. V. For the three-momentum distribution the authors of Ref. [70] have included further corrections to account for the difference with the results of the correct Schrödinger equation (55). In Refs. [69,70] the "potential-subtracted" mass has been tested in different ways as an alternative mass parameter for the total cross section. As far as the uncertainties in the top mass determination at a future linear collider are concerned, Ref. [69] arrives at conclusions similar to ours. In Ref. [71] the techniques used in Refs. [17,11] have been employed to calculate the total cross section, the angular distribution and the top-quark polarization for top-quark pair production close to threshold in $e^{+} e^{-}$and $\gamma \gamma$ collisions. The corrections originating from the higher-order contributions in the Coulomb potential have been calculated analytically. In Refs. [69-71] the top-quark width has been implemented by the replacement rule ' $E \rightarrow E+i \Gamma_{t}$,', where $E$ is the centerof-mass energy with respect to two times the top-quark mass.

\section{ACKNOWLEDGMENTS}

We thank M. Beneke for discussions and M. Beneke, Z. Ligeti, and A. V. Manohar for reading the manuscript. The
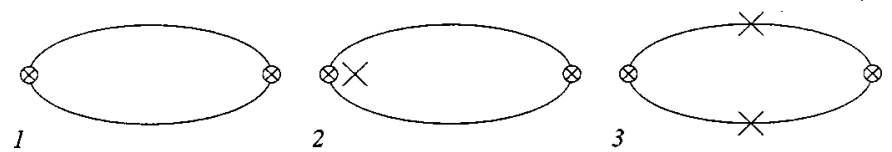

FIG. 14. Graphical representation of the NRQCD vector-current correlators diagrams needed to determine the nonrelativistic vectorcurrent-induced cross section at the Born level and NNLO in the nonrelativistic expansion. 

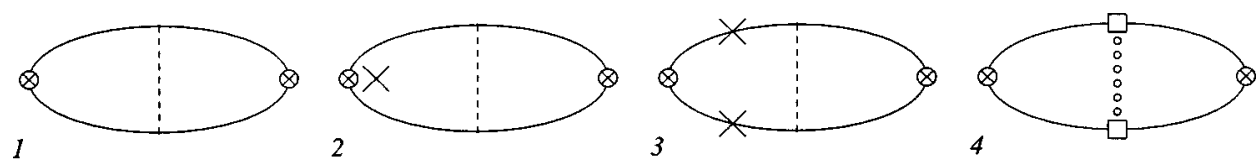

FIG. 15. Graphical representation of the NRQCD vector-current correlators diagrams needed to determine the nonrelativistic vector current induced cross section at $\mathcal{O}\left(\alpha_{s}\right)$ and NNLO in the nonrelativistic expansion.

work of A.H.H. is in part supported by the EU Fourth Framework Program "Training and Mobility of Researchers,' Network 'Quantum Chromodynamics and Deep Structure of Elementary Particles,' Contract No. FMRX-CT980194 (DG12-MIHT).

\section{APPENDIX: CALCULATION OF THE SHORT-DISTANCE COEFFICIENT $C^{v}$}

In this appendix we present details of the calculation of the short-distance coefficient $C^{\mathrm{v}}$ to order $\alpha_{s}^{2}$, assuming that the top quarks are stable $\left(\Gamma_{t}=0\right)$. We recall that $C^{\mathrm{v}}$ is the square of the short-distance coefficient $c_{1}^{\mathrm{v}}$ of the ${ }^{3} S_{1}$ NRQCD current $\tilde{\psi}^{\dagger} \boldsymbol{\sigma} \tilde{\chi}$ [see Eq. (2)]; $c_{1}^{\mathrm{v}}$ contains those contributions in the vector-current-induced top-quark-topantiquark production diagrams, which come from loop momenta $p=\left(p^{0}, \vec{p}\right)$ with $|\vec{p}|>\Lambda$ for $\sqrt{q^{2}}=2 M_{t}$. As explained in Sec. IV, we have to determine $C^{\mathrm{V}}$ by employing the specific routing convention shown in Fig. 1. In principle, it would be possible to determine $C^{\vee}$ by calculating the diagrams for the vector-current-induced cross section in full QCD restricting the loop momenta such that the spatial components would be larger than $\Lambda$. However, in a cutoff scheme it is more economical to first calculate the vector-currentinduced cross section in NRQCD up to order $\alpha_{s}^{2}$ and NNLO in the velocity expansion and then to adjust the coefficients of $C^{\mathrm{V}}$ such that the cross section in NRQCD is equal to the cross section in full QCD, likewise calculated to order $\alpha_{s}^{2}$ and NNLO in the velocity expansion.

The expression of the total vector-current-induced cross section in full QCD at order $\alpha_{s}^{2}$ and NNLO in the velocity expansion reads $\left[a \equiv C_{F} \alpha_{s}(\mu)\right]$

$$
\begin{aligned}
R_{2 \text { loop QCD }}^{\mathrm{V}, \mathrm{NNLO}}= & N_{c}\left(\left[\frac{3}{2} \frac{p_{0}}{M_{t}}-\frac{5}{4} \frac{p_{0}^{3}}{M_{t}^{3}}\right]+\frac{a}{\pi}\left[\frac{3 \pi^{2}}{4}-6 \frac{p_{0}}{M_{t}}+\frac{\pi^{2}}{2} \frac{p_{0}^{2}}{M_{t}^{2}}\right]\right. \\
& +a^{2}\left\{\frac{\pi^{2} M_{t}}{8 p_{0}}-\frac{3}{2}\left[2+\frac{1}{8 C_{F}}\left(\beta_{0} \ln \frac{4 p_{0}^{2}}{\mu_{\text {hard }}^{2}}-a_{1}\right)\right]\right. \\
& +\left[\frac{13 \pi^{2}}{48}+\frac{3}{2 C_{F}^{2}} \kappa+\frac{3 \beta_{0}}{2 C_{F} \pi^{2}} \ln \frac{M_{t}^{2}}{\mu_{\text {hard }}^{2}}\right. \\
& \left.\left.\left.-\left(1+\frac{3}{2} \frac{C_{A}}{C_{F}}\right) \ln \left(\frac{p_{0}}{M_{t}}\right)\right] \frac{p_{0}}{M_{t}}\right\}\right),
\end{aligned}
$$

where

$$
\begin{aligned}
\kappa= & C_{F}^{2}\left[\frac{1}{\pi^{2}}\left(\frac{39}{4}-\zeta_{3}\right)+\frac{4}{3} \ln 2-\frac{35}{18}\right] \\
& -C_{A} C_{F}\left[\frac{1}{\pi^{2}}\left(\frac{151}{36}+\frac{13}{2} \zeta_{3}\right)+\frac{8}{3} \ln 2-\frac{179}{72}\right] \\
& +C_{F} T\left[\frac{4}{9}\left(\frac{11}{\pi^{2}}-1\right)\right]+C_{F} T n_{l}\left[\frac{11}{9 \pi^{2}}\right] .
\end{aligned}
$$

The Born and $\mathcal{O}\left(\alpha_{s}\right)$ [72] contributions are standard. At order $\alpha_{s}^{2}$ the contributions in Eq. (A1) that are proportional to $C_{F}^{2}, C_{A} C_{F}, C_{F} T n_{l}$, and $C_{F} T$ have been calculated in Refs. $[73,35,63,74]$, and $[63,75]$, respectively. (See also Refs. $[68,76]$.

To determine the corresponding total vector-currentinduced cross section in NRQCD, we have to calculate the absorptive part of the correlator diagrams depicted in Figs. 14, 15, and 16. The various symbols are defined in Fig. 17. We emphasize that we neglect multiple insertions of NNLO contributions.

The results for the absorptive parts of the individual dia$\operatorname{grams} \operatorname{read}\left[a \equiv C_{F} \alpha_{s}, D(\boldsymbol{k}) \equiv M_{t} /\left(\boldsymbol{k}^{2}-p_{0}^{2}-i \boldsymbol{\epsilon}\right)\right]$

$$
\begin{gathered}
I_{1}^{(0)}=\operatorname{Im}\left[\int \frac{d^{3} \boldsymbol{k}}{(2 \pi)^{3}} D(\boldsymbol{k})\right]=\frac{M_{t}^{2}}{4 \pi} \frac{p_{0}}{M_{t}}, \\
I_{2}^{(0)}=\operatorname{Im}\left[2 \int \frac{d^{3} \boldsymbol{k}}{(2 \pi)^{3}}\left(-\frac{\boldsymbol{k}^{2}}{6 M_{t}^{2}}\right) D(\boldsymbol{k})\right]=-\frac{M_{t}^{2}}{4 \pi} \frac{p_{0}^{3}}{3 M_{t}^{3}}, \\
I_{3}^{(0)}=\operatorname{Im}\left[\int \frac{d^{3} \boldsymbol{k}}{(2 \pi)^{3}}\left(\frac{\boldsymbol{k}^{2}+p_{0}^{2}}{4 M_{t}^{2}}\right) D(\boldsymbol{k})\right]=\frac{M_{t}^{2}}{4 \pi} \frac{p_{0}^{3}}{2 M_{t}^{3}}, \\
I_{1}^{(1)}=\operatorname{Im}\left[\int \frac{d^{3} \boldsymbol{k}_{1}}{(2 \pi)^{3}} \int \frac{d^{3} \boldsymbol{k}_{2}}{(2 \pi)^{3}} D\left(\boldsymbol{k}_{1}\right) \frac{4 \pi a}{\left(\boldsymbol{k}_{1}-\boldsymbol{k}_{2}\right)^{2}} D\left(\boldsymbol{k}_{2}\right)\right]=\frac{a M_{t}^{2}}{4 \pi^{2}}\left[\frac{\pi^{2}}{2}-\frac{4 p_{0}}{\Lambda}+\mathcal{O}\left(\frac{p_{0}^{3}}{M_{t}^{3}}\right)\right],
\end{gathered}
$$



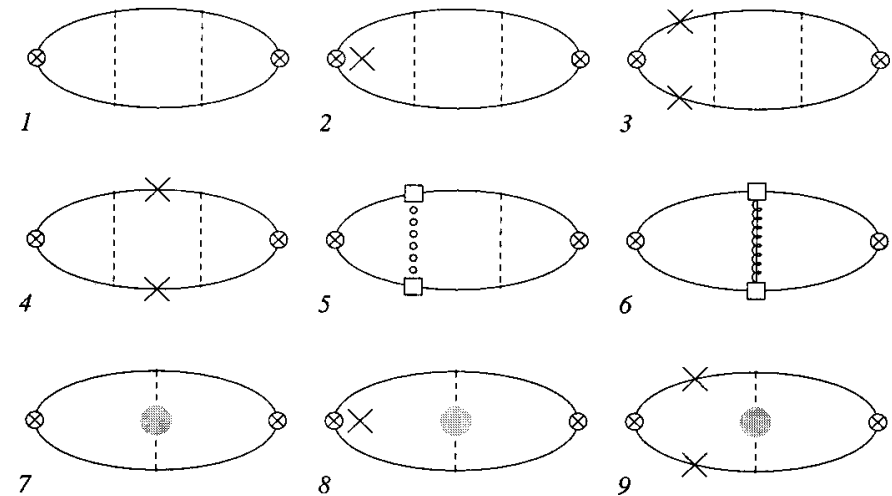

FIG. 16. Graphical representation of the NRQCD vector-current correlators diagrams needed to determine the nonrelativistic vectorcurrent-induced cross section at $\mathcal{O}\left(\alpha_{s}^{2}\right)$ and NNLO in the nonrelativistic expansion.

$$
\begin{aligned}
& I_{2}^{(1)}=\operatorname{Im}\left[2 \int \frac{d^{3} \boldsymbol{k}_{1}}{(2 \pi)^{3}} \int \frac{d^{3} \boldsymbol{k}^{2}}{(2 \pi)^{3}}\left(-\frac{\boldsymbol{k}_{1}^{2}}{6 M_{t}^{2}}\right) D\left(\boldsymbol{k}_{1}\right) \frac{4 \pi a}{\left(\boldsymbol{k}_{1}-\boldsymbol{k}_{2}\right)^{2}} D\left(\boldsymbol{k}_{2}\right)\right]=-\frac{a M_{t}^{2}}{4 \pi^{2}}\left[\frac{2 \Lambda p_{0}}{3 M_{t}^{2}}+\frac{p_{0}^{2} \pi^{2}}{6 M_{t}^{2}}+\mathcal{O}\left(\frac{p_{0}^{3}}{M_{t}^{3}}\right)\right] \\
& I_{3}^{(1)}=\operatorname{Im}\left[2 \int \frac{d^{3} \boldsymbol{k}_{1}}{(2 \pi)^{3}} \int \frac{d^{3} \boldsymbol{k}_{2}}{(2 \pi)^{3}}\left(\frac{\boldsymbol{k}_{1}^{2}+p_{0}^{2}}{4 M_{t}^{2}}\right) D\left(\boldsymbol{k}_{1}\right) \frac{4 \pi a}{\left(\boldsymbol{k}_{1}-\boldsymbol{k}_{2}\right)^{2}} D\left(\boldsymbol{k}_{2}\right)\right]=\frac{a M_{t}^{2}}{4 \pi^{2}}\left[\frac{\Lambda p_{0}}{M_{t}^{2}}+\frac{p_{0}^{2} \pi^{2}}{2 M_{t}^{2}}+\mathcal{O}\left(\frac{p_{0}^{3}}{M_{t}^{3}}\right)\right] \\
& I_{4}^{(1)}=\operatorname{Im}\left[\int \frac{d^{3} \boldsymbol{k}_{1}}{(2 \pi)^{3}} \int \frac{d^{3} \boldsymbol{k}_{2}}{(2 \pi)^{3}} D\left(\boldsymbol{k}_{1}\right)\left(2 \frac{\pi a}{M_{t}^{2}} \frac{\boldsymbol{k}_{1}{ }^{2}+\boldsymbol{k}_{2}{ }^{2}}{\left(\boldsymbol{k}_{1}-\boldsymbol{k}_{2}\right)^{2}}-\frac{11}{3} \frac{\pi a}{M_{t}^{2}}\right) D\left(\boldsymbol{k}_{2}\right)\right]=\frac{a M_{t}^{2}}{4 \pi^{2}}\left[-\frac{5 \Lambda p_{0}}{3 M_{t}^{2}}+\frac{p_{0}^{2} \pi^{2}}{2 M_{t}^{2}}+\mathcal{O}\left(\frac{p_{0}^{3}}{M_{t}^{3}}\right)\right], \\
& I_{1}^{(2)}=\operatorname{Im}\left[\int \frac{d^{3} \boldsymbol{k}_{1}}{(2 \pi)^{3}} \int \frac{d^{3} \boldsymbol{k}_{2}}{(2 \pi)^{3}} \int \frac{d^{3} \boldsymbol{k}_{3}}{(2 \pi)^{3}} D\left(\boldsymbol{k}_{1}\right) \frac{4 \pi_{a}}{\left(\boldsymbol{k}_{1}-\boldsymbol{k}_{a}\right)^{2}} D\left(\boldsymbol{k}_{2}\right) \frac{4 \pi a}{\left(\boldsymbol{k}_{2}-\boldsymbol{k}_{3}\right)^{2}} D\left(\boldsymbol{k}_{3}\right)\right] \\
& =\frac{a^{2} M_{t}^{2}}{4 \pi^{3}}\left[\frac{M_{t} \pi^{4}}{12 p_{0}}-\frac{2 M_{t} \pi^{2}}{\Lambda}+\frac{M_{t} p_{0}\left(12-\pi^{2}\right)}{2 \Lambda^{2}}+\mathcal{O}\left(\frac{p_{0}^{2}}{M_{t}^{2}}\right)\right] \text {, } \\
& I_{2}^{(2)}=\operatorname{Im}\left[2 \int \frac{d^{3} \boldsymbol{k}_{1}}{(2 \pi)^{3}} \int \frac{d^{3} \boldsymbol{k}_{2}}{(2 \pi)^{3}} \int \frac{d^{3} \boldsymbol{k}_{3}}{(2 \pi)^{3}}\left(-\frac{\boldsymbol{k}_{1}^{2}}{6 M_{t}^{2}}\right) D\left(\boldsymbol{k}_{1}\right) \frac{4 \pi a}{\left(\boldsymbol{k}_{1}-\boldsymbol{k}_{2}\right)^{2}} D\left(\boldsymbol{k}_{2}\right) \frac{4 \pi a}{\left(\boldsymbol{k}_{2}-\boldsymbol{k}_{3}\right)^{2}} D\left(\boldsymbol{k}_{3}\right)\right] \\
& =-\frac{a^{2} M_{t}^{2}}{4 \pi^{3}}\left[\frac{\Lambda \pi^{2}}{3 M_{t}}-\frac{p_{0}\left(84+3 \pi^{2}-\pi^{4}\right)}{36 M_{t}}+\mathcal{O}\left(\frac{p_{0}^{2}}{M_{t}^{2}}\right)\right] \text {, }
\end{aligned}
$$
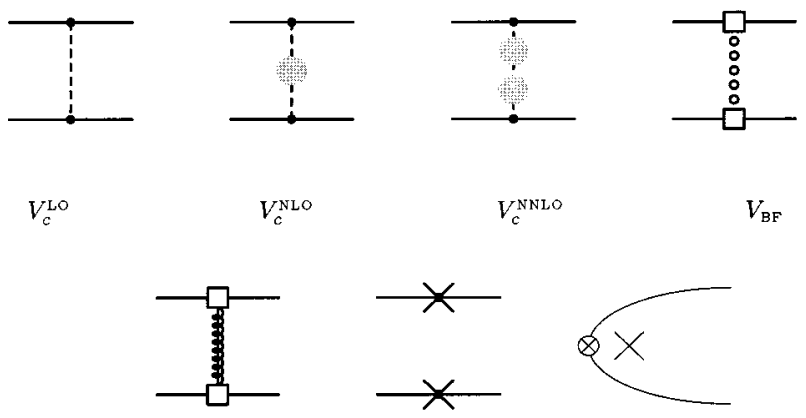

FIG. 17. Symbols describing the interactions potentials $V_{c}^{\mathrm{LO}}, V_{c}^{\mathrm{NLO}}, V_{c}^{\mathrm{NNLO}}, V_{\mathrm{BF}}$, and $V_{\mathrm{NA}}$ and the kinetic energy correction $\delta H_{\mathrm{kin}}$ $=\left(p_{0}^{4}-\vec{k}^{4}\right) / 4 M_{t}^{3} . V_{c}^{\mathrm{LO}}, V_{c}^{\mathrm{NLO}}$, and $V_{c}^{\mathrm{NLO}}$ refer to the Born, one-loop, and two-loop contributions to the Coulomb potential presented in Eq. (22). 


$$
\begin{aligned}
& I_{3}^{(2)}=\operatorname{Im}\left[2 \int \frac{d^{3} \boldsymbol{k}_{1}}{(2 \pi)^{3}} \int \frac{d^{3} \boldsymbol{k}_{2}}{(2 \pi)^{3}} \int \frac{d^{3} \boldsymbol{k}_{3}}{(2 \pi)^{3}}\left(\frac{\boldsymbol{k}_{1}^{2}+p_{0}^{2}}{4 M_{t}^{2}}\right) D\left(\boldsymbol{k}_{1}\right) \frac{4 \pi a}{\left(\boldsymbol{k}_{1}-\boldsymbol{k}_{2}\right)^{2}} D\left(\boldsymbol{k}_{2}\right) \frac{4 \pi a}{\left(\boldsymbol{k}_{2}-\boldsymbol{k}_{3}\right)^{2}} D\left(\boldsymbol{k}_{3}\right)\right] \\
& =\frac{a^{2} M_{t}^{2}}{4 \pi^{3}}\left[\frac{\Lambda \pi^{2}}{2 M_{t}}-\frac{p_{0}\left(84+3 \pi^{2}-2 \pi^{4}\right)}{24 M_{t}}+\mathcal{O}\left(\frac{p_{0}^{2}}{M_{t}^{2}}\right)\right], \\
& I_{4}^{(2)}=\operatorname{Im}\left[\int \frac{d^{3} \boldsymbol{k}_{1}}{(2 \pi)^{3}} \int \frac{d^{3} \boldsymbol{k}_{2}}{(2 \pi)^{3}} \int \frac{d^{3} \boldsymbol{k}_{3}}{(2 \pi)_{3}} D\left(\boldsymbol{k}_{1}\right) \frac{4 \pi a}{\left(\boldsymbol{k}_{1}-\boldsymbol{k}_{2}\right)^{2}}\left(\frac{\boldsymbol{k}_{2}^{2}+p_{0}^{2}}{4 M_{t}^{2}}\right) D\left(\boldsymbol{k}_{2}\right) \frac{4 \pi a}{\left(\boldsymbol{k}_{2}-\boldsymbol{k}_{3}\right)^{2}} D\left(\boldsymbol{k}_{3}\right)\right] \\
& =\frac{a^{2} M_{t}^{2}}{4 \pi^{3}}\left\{\frac{p_{0}}{24 M_{t}}\left[12 \pi^{2}+\pi^{4}-42 \zeta_{3}-12 \pi^{2} \ln \left(\frac{2 p_{0}}{\Lambda}\right)\right]+\mathcal{O}\left(\frac{p_{0}^{2}}{M_{t}^{2}}\right)\right\}, \\
& I_{5}^{(2)}=\operatorname{Im}\left[2 \int \frac{d^{3} \boldsymbol{k}_{1}}{(2 \pi)^{3}} \int \frac{d^{3} \boldsymbol{k}_{2}}{(2 \pi)^{3}} \int \frac{d^{3} \boldsymbol{k}_{3}}{(2 \pi)^{3}} D\left(\boldsymbol{k}_{1}\right)\left(2 \frac{\pi a}{M_{t}^{2}} \frac{\boldsymbol{k}_{1}^{2}+\boldsymbol{k}_{2}^{2}}{\left(\boldsymbol{k}_{1}-\boldsymbol{k}_{2}\right)^{2}}-\frac{11}{3} \frac{\pi a}{M_{t}^{2}}\right) D\left(\boldsymbol{k}_{2}\right) \frac{4 \pi a}{\left(\boldsymbol{k}_{2}-\boldsymbol{k}_{3}\right)^{2}} D\left(\boldsymbol{k}_{3}\right)\right] \\
& =\frac{a^{2} M_{t}^{2}}{4 \pi^{3}}\left\{-\frac{5 \Lambda \pi^{2}}{6 M_{t}}+\frac{p_{0}}{12 M_{t}}\left[92+21 \pi^{2}+2 \pi^{4}-7 \zeta_{3}-2 \pi^{2} \ln \left(\frac{2 p_{0}}{\Lambda}\right)\right]+\mathcal{O}\left(\frac{p_{0}^{2}}{M_{t}^{2}}\right)\right\}, \\
& I_{6}^{(2)}=\operatorname{Im}\left[\int \frac{d^{3} \boldsymbol{k}_{1}}{(2 \pi)^{3}} \int \frac{d^{3} \boldsymbol{k}_{2}}{(2 \pi)^{3}} D\left(\boldsymbol{k}_{1}\right)\left(\frac{C_{A}}{C_{F}} \frac{\pi^{2} a^{2}}{M_{t}\left|\boldsymbol{k}_{1}-\boldsymbol{k}_{2}\right|}\right) D\left(\boldsymbol{k}_{2}\right)\right]=-\frac{a^{2} M_{t}^{2}}{4 \pi^{3}}\left\{\frac{C_{A} p_{0} \pi^{2}}{C_{F} M_{t}}\left[-1+\ln \left(\frac{2 p_{0}}{\Lambda}\right)\right]+\mathcal{O}\left(\frac{p_{0}^{2}}{M_{t}^{2}}\right)\right\}, \\
& I_{7}^{(2)}=\operatorname{Im}\left\{\int \frac{d^{3} \boldsymbol{k}_{1}}{(2 \pi)^{3}} \int \frac{d^{3} \boldsymbol{k}^{2}}{(2 \pi)^{3}} D\left(\boldsymbol{k}_{1}\right) \frac{4 \pi a}{\left(\boldsymbol{k}_{2}-\boldsymbol{k}_{3}\right)^{2}} \frac{a}{4 C_{F} \pi}\left[-\beta_{0} \ln \left(\frac{\left(\boldsymbol{k}_{1}-\boldsymbol{k}_{2}\right)^{2}}{\mu^{2}}\right)+a_{1}\right] D\left(\boldsymbol{k}_{2}\right)\right\} \\
& =\frac{a^{2} M_{t}^{2}}{4 C_{F} \pi^{3}}\left(\beta_{0}\left\{-\frac{\pi^{2}}{4} \ln \left(\frac{2 p_{0}}{\mu}\right)+\frac{2 p_{0}}{\Lambda}\left[1+\ln \left(\frac{\Lambda}{\mu}\right)\right]\right\}+a_{1}\left(\frac{\pi^{2}}{8}-\frac{p_{0}}{\Lambda}\right)+\mathcal{O}\left(\frac{p_{0}^{2}}{M_{t}^{2}}\right)\right), \\
& I_{8}^{(2)}=\operatorname{Im}\left\{2 \int \frac{d^{3} \boldsymbol{k}_{1}}{(2 \pi)^{3}} \int \frac{d^{3} \boldsymbol{k}_{2}}{(2 \pi)^{3}}\left(-\frac{\boldsymbol{k}_{1}^{2}}{6 M_{t}^{2}}\right) D\left(\boldsymbol{k}_{1}\right) \frac{4 \pi a}{\left(\boldsymbol{k}_{2}-\boldsymbol{k}_{3}\right)^{2}} \frac{a}{4 C_{F} \pi}\left[-\beta_{0} \ln \left(\frac{\left(\boldsymbol{k}_{1}-\boldsymbol{k}_{2}\right)^{2}}{\mu^{2}}\right)+a_{1}\right] D\left(\boldsymbol{k}_{2}\right)\right\} \\
& =\frac{a^{2} M_{t}^{2}}{4 C_{F} \pi^{3}}\left(\beta_{0}\left\{\frac{\Lambda p_{0}}{3 M_{t}^{2}}\left[-1+\ln \left(\frac{\Lambda}{\mu}\right)\right]\right\}-a_{1} \frac{\Lambda p_{0}}{6 M_{t}^{2}}+\mathcal{O}\left(\frac{p_{0}^{2}}{M_{t}^{2}}\right)\right), \\
& I_{9}^{(2)}=\operatorname{Im}\left\{2 \int \frac{d^{3} \boldsymbol{k}_{1}}{(2 \pi)^{3}} \int \frac{d^{3} \boldsymbol{k}_{2}}{(2 \pi)^{3}}\left(\frac{\boldsymbol{k}_{1}^{2}+p_{0}^{2}}{4 M_{t}^{2}}\right) D\left(\boldsymbol{k}_{1}\right) \frac{4 \pi a}{\left(\boldsymbol{k}_{2}-\boldsymbol{k}_{3}\right)^{2}} \frac{a}{4 C_{F} \pi}\left[-\beta_{0} \ln \left(\frac{\left(\boldsymbol{k}_{1}-\boldsymbol{k}_{2}\right)^{2}}{\mu^{2}}\right)+a_{1}\right] D\left(\boldsymbol{k}_{2}\right)\right\} \\
& =\frac{a^{2} M_{t}^{2}}{4 C_{F} \pi^{3}}\left(\beta_{0}\left\{\frac{\Lambda p_{0}}{2 M_{t}^{2}}\left[1-\ln \left(\frac{\Lambda}{\mu}\right)\right]\right\}+a_{1} \frac{\Lambda p_{0}}{4 M_{t}^{2}}+\mathcal{O}\left(\frac{p_{0}^{2}}{M_{t}^{2}}\right)\right),
\end{aligned}
$$

where the upper index of the functions $I_{j}^{(i)}$ corresponds to the power of the strong coupling of the diagrams and the lower index to the numeration given in Figs. 14, 15, and 16. Combinatorial factors are taken into account. We note that the results above have been expanded in $p_{0} / M_{t}, p_{0} / \Lambda \ll 1$; no condition has been assumed for the ratio $\Lambda / M_{t}$.

Summing all terms leads to the total vector-currentinduced cross section in NRQCD:

$$
R_{\mathrm{NNLO}}^{\mathrm{v} \text { thr }}=\frac{6 \pi N_{c}}{M_{t}^{2}\left(1+\frac{p_{0}^{2}}{M_{t}^{2}}\right)} C^{\mathrm{v}}\left[\sum_{i=1}^{3} I_{i}^{(0)}+\sum_{i=1}^{4} I_{i}^{(1)}+\sum_{i=1}^{9} I_{i}^{(2)}\right]
$$

The short-distance coefficient $C^{\mathrm{v}}$ has to be chosen such that the RHS of Eqs. (A1) and (A19) are equal for all terms up to 
TABLE III. The NLO and NNLO contributions to the short-distance coefficient $C^{\mathrm{v}}$ in our cutoff scheme and in the $\overline{\mathrm{MS}}$ scheme for various choices of the cutoff $\Lambda$ and the renormalization scale $\mu$. We have chosen $\alpha_{s}\left(M_{z}\right)=0.118$, and two-loop running for the strong coupling has been employed.

\begin{tabular}{|c|c|c|c|c|c|c|c|c|}
\hline \multirow[t]{2}{*}{$\mu[\mathrm{GeV}]$} & \multicolumn{3}{|c|}{$c_{\mathrm{NLO}}^{\mathrm{v}}(\Lambda[\mathrm{GeV}])$} & \multirow[t]{2}{*}{$c \frac{\mathrm{v}}{\mathrm{MS}, \mathrm{NLO}}$} & \multicolumn{3}{|c|}{$c_{\mathrm{NNLO}}^{\mathrm{v}}(\Lambda[\mathrm{GeV}])$} & \multirow[t]{2}{*}{$c_{\overline{\mathrm{MS}}, \mathrm{NNLO}}^{\mathrm{v}}$} \\
\hline & 90 & 175 & 350 & & 90 & 175 & 350 & \\
\hline 15 & 0.261 & 0 & -0.138 & -0.276 & -0.018 & -0.101 & -0.040 & 0.128 \\
\hline 30 & 0.228 & 0 & -0.120 & -0.241 & 0.018 & -0.069 & -0.029 & 0.025 \\
\hline 60 & 0.202 & 0 & -0.107 & -0.214 & 0.039 & -0.048 & -0.022 & 0.037 \\
\hline 175 & 0.172 & 0 & -0.091 & -0.182 & 0.056 & -0.028 & -0.015 & -0.091 \\
\hline
\end{tabular}

order $\alpha_{s}^{2}$ and NNLO in the non-relativistic expansion. The result reads

$$
C^{\mathrm{v}}(\Lambda, \mu)=1+c_{\mathrm{NLO}}^{\mathrm{v}}(\Lambda, \mu)+c_{\mathrm{NNLO}}^{\mathrm{v}}(\Lambda, \mu),
$$

where

$$
\begin{aligned}
c_{\mathrm{NLO}}^{\mathrm{v}}(\Lambda, \mu)= & \frac{4 \alpha}{\pi}\left[-1+\frac{M_{t}}{\Lambda}\right], \\
c_{\mathrm{NNLO}}^{\mathrm{v}}(\Lambda, \mu)= & \frac{4 \alpha \Lambda}{3 \pi M_{t}}+\frac{a^{2}}{\pi^{2}}\left\{\frac { \beta _ { 0 } } { C _ { F } } \left[-\frac{\Lambda^{2}+12 M_{t}^{2}}{6 \Lambda M_{t}}\right.\right. \\
& \left.+\frac{\Lambda^{2}-12 M_{t}^{2}}{6 \Lambda M_{t}} \ln \left(\frac{\Lambda}{\mu}\right)+2 \ln \left(\frac{M_{t}}{\mu}\right)\right] \\
& -\frac{a_{1}}{C_{F}} \frac{\Lambda^{2}-12 M_{t}^{2}}{12 \Lambda M_{t}}+\pi^{2}\left(\frac{2}{3}+\frac{C_{A}}{C_{F}}\right) \ln \left(2 \frac{M_{t}}{\Lambda}\right) \\
& +\frac{\pi^{2} \kappa}{C_{F}^{2}}+\frac{16 \Lambda^{2}}{9 M_{t}^{2}}-\frac{16 \Lambda}{3 M_{t}}-\frac{16 M_{t}}{\Lambda} \\
& +\frac{M_{t}^{2}\left(20+\pi^{2}\right)}{2 \Lambda^{2}}-\frac{53 \pi^{2}}{24}-\frac{C_{A} \pi^{2}}{C_{F}} \\
& \left.+\frac{25}{6}+\frac{7}{3} \zeta_{3}\right\} .
\end{aligned}
$$

current in the nonrelativistic current correlators. Thus it is important to consider this term as NNLO. It is a conspicuous fact that the NLO short-distance coefficient $c_{\mathrm{NLO}}^{\mathrm{v}}$ vanishes for the choice $\Lambda=M_{t}$. We emphasize, however, that this cancellation is purely accidental. Nevertheless, comparing the short-distance constant $C^{\mathrm{v}}$ calculated in our cutoff regularization scheme with the corresponding coefficient obtained in the $\overline{\mathrm{MS}}$ scheme $[35,36]$

$$
C \overline{\mathrm{MS}}(\mu)=1+c \frac{\mathrm{v}}{\mathrm{MS}, \mathrm{NLO}}(\Lambda, \mu)+c \frac{\mathrm{v}}{\mathrm{MS}, \mathrm{NNLO}}(\Lambda, \mu),
$$

where $\left[\alpha_{s} \equiv \alpha_{s}(\mu)\right]$

$$
\begin{aligned}
c \stackrel{\mathrm{vS}, \mathrm{NLO}}{ }(\mu)= & -4 C_{F} \frac{\alpha_{s}}{\pi}, \\
c \frac{\mathrm{v}}{\mathrm{MS}, \mathrm{NNLO}}(\mu)= & \frac{\alpha_{s}^{2}}{\pi^{2}}\left\{C _ { F } ^ { 2 } \left[\frac{39}{4}-\frac{79 \pi^{2}}{18}+2 \pi^{2} \ln 2\right.\right. \\
& \left.+\frac{\pi^{2}}{3} \ln \left(\frac{M_{t}^{2}}{\mu^{2}}\right)-\zeta_{3}\right] \\
& +C_{A} C_{F}\left[-\frac{151}{36}+\frac{89 \pi^{2}}{72}-\frac{5 \pi^{2}}{3} \ln 2\right. \\
& \left.+\frac{\pi^{2}}{2} \ln \left(\frac{M_{t}^{2}}{\mu^{2}}\right)-\frac{13}{2} \zeta_{3}\right] \\
& \left.+C_{F} T\left(\frac{44}{9}-\frac{4 \pi^{2}}{9}\right)+C_{F} T n_{f} \frac{11}{9}\right\},
\end{aligned}
$$
in Eq. (A22) contains the term $\left(4 \alpha_{s} / 3 \pi\right)\left(\Lambda / M_{t}\right)$, which is of order $\alpha_{s}$ only. This term is a manifestation of the powercounting breaking effects discussed in Sec. IV. The term exists because it subtracts the power-counting breaking terms originating from the linear UV-divergent behavior of the Breit-Fermi potential $V_{\mathrm{BF}}$, the kinetic energy correction $\left(p_{0}^{4}-\boldsymbol{k}^{4}\right) /\left(4 M_{t}^{3}\right)$, and the dimension-5 NRQCD vector- 
strong coupling. We are not aware of any principle reason why the short-distance corrections should be, in general, better convergent in our cutoff scheme than when using the $\overline{\mathrm{MS}}$ regularization. We finally note that the potentially large logarithmic term $\alpha_{s}^{2} C_{A} C_{F} \ln \left(M_{t} / \mu\right)$ in $C_{\overline{\mathrm{MS}}}^{\mathrm{v}}$, which corresponds to an anomalous dimension of the dimension-3 NRQCD vector-current $\widetilde{\psi}^{\dagger} \boldsymbol{\sigma} \tilde{\chi}$, does not exist in $C^{\vee}$, since in our cutoff scheme the corresponding logarithmic divergence in the
NRQCD diagrams is cut off at the scale $\Lambda \sim M_{t}$ rather than $\mu \sim M_{t} \mathrm{v}$, as in the $\overline{\mathrm{MS}}$ scheme. However, we emphasize that the absence of this logarithmic term in $C^{\mathrm{v}}$ is traded for the existence of logarithms of the ratio $2 p_{0} / \Lambda$ in the nonrelativistic correlator, which are not present in the $\overline{\mathrm{MS}}$ scheme. The logarithms of $\Lambda / \mu$ and $M_{t} / \mu$ in $c_{\mathrm{NNLO}}^{v}$, Eq. (A22), originate from the running of the strong coupling and are not related to an anomalous dimension.
[1] R. Patridge, Report No. FERMILAB-CONF-98-375, hep-ex/9811035; talk given at 29th International Conference on High-Energy Physics (ICHEP 98), Vancouver, Canada, 1998.

[2] V. S. Fadin and V. A. Khoze, Pis'ma Zh. Eksp. Teor. Fiz. 46, 417 (1987) [JETP Lett. 46, 525 (1987)].

[3] E. C. Poggio, H. R. Quinn, and S. Weinberg, Phys. Rev. D 13, 1958 (1976).

[4] P. Comas, R. Miquel, M. Martinez, and S. Orteu, talk given at Physics with $e^{+} e^{-}$Linear Colliders, 1995, $e^{+} e^{-}$Linear Collisions at $500 \mathrm{GeV}, 1995$, Report No. DESY 92-123D, p. 57.

[5] V. S. Fadin and V. A. Khoze, Yad. Fiz. 48, 487 (1988) [Sov. J. Nucl. Phys. 48, 309 (1988)].

[6] M. J. Strassler and M. E. Peskin, Phys. Rev. D 43, 1500 (1991).

[7] W. Kwong, Phys. Rev. D 43, 1488 (1991).

[8] M. Jeżabek, J. H. Kühn, and T. Teubner, Z. Phys. C 56, 653 (1992).

[9] Y. Sumino, K. Fujii, K. Hagiwara, H. Murayama, and C.-K. Ng, Phys. Rev. D 47, 56 (1993).

[10] K. Fujii, T. Matsui, and Y. Sumino, Phys. Rev. D 50, 4341 (1994).

[11] A. H. Hoang and T. Teubner, Phys. Rev. D 58, 114023 (1998).

[12] K. Melnikov and A. Yelkhovsky, Nucl. Phys. B528, 59 (1998).

[13] O. Yakovlev, Phys. Lett. B 457, 170 (1999).

[14] W. E. Caswell and G. E. Lepage, Phys. Lett. 167B, 437 (1986).

[15] G. T. Bodwin, E. Braaten, and G. P. Lepage, Phys. Rev. D 51, 1125 (1995); 55, 5853 (1997).

[16] A. H. Hoang, Phys. Rev. D 57, 1615 (1998).

[17] A. H. Hoang, Phys. Rev. D 56, 5851 (1997).

[18] V. S. Fadin, V. A. Khoze, and A. D. Martin, Phys. Rev. D 49, 2247 (1994).

[19] V. S. Fadin, V. A. Khoze, and A. D. Martin, Phys. Lett. B 320, 141 (1994).

[20] K. Melnikov and O. Yakovlev, Phys. Lett. B 324, 217 (1994).

[21] R. Harlander, M. Jezabek, J. H. Kühn, and M. Peter, Z. Phys. C 73, 477 (1997).

[22] M. Peter and Y. Sumino, Phys. Rev. D 57, 6912 (1998).

[23] J. H. Kühn and T. Teubner, Eur. Phys. J. C 9, 221 (1999).

[24] A. Pineda and J. Soto, Nucl. Phys. B (Proc. Suppl.) 64, 428 (1998).

[25] M. Beneke and V. A. Smirnov, Nucl. Phys. B522, 321 (1998).

[26] W. Fischler, Nucl. Phys. B129, 157 (1977).

[27] A. Billoire, Phys. Lett. 92B, 343 (1980).

[28] Y. Schröder, Phys. Lett. B 447, 321 (1999).
[29] M. Peter, Phys. Rev. Lett. 78, 602 (1997); Nucl. Phys. B501, 471 (1997).

[30] S. N. Gupta and S. F. Radford, Phys. Rev. D 24, 2309 (1981); 25, 3430(E) (1982).

[31] S. N. Gupta, S. F. Radford, and W. W. Repko, Phys. Rev. D 26, 3305 (1982).

[32] W. Kummer and W. Mödritsch, Z. Phys. C 66, 225 (1995).

[33] H. A. Bethe and E. E. Salpeter, Quantum Mechanics of Oneand Two-Electron Atoms (Academic, New York, 1957).

[34] A. H. Hoang, Phys. Rev. D 59, 014039 (1999).

[35] A. Czarnecki and K. Melnikov, Phys. Rev. Lett. 80, 2531 (1998).

[36] M. Beneke, A. Signer, and V. A. Smirnov, Phys. Rev. Lett. 80, 2535 (1998).

[37] M. Beneke and A. H. Hoang (in preparation).

[38] M. Jeżabek and J. H. Kühn, Nucl. Phys. B314, 1 (1989).

[39] A. Czarnecki and K. Melnikov, Nucl. Phys. B544, 520 (1999).

[40] A. Denner and T. Sack, Nucl. Phys. B358, 46 (1991).

[41] R. J. Guth and J. H. Kühn, Nucl. Phys. B368, 38 (1992).

[42] E. H. Wichmann and C. H. Woo, J. Math. Phys. 2, 178 (1961).

[43] L. Hostler, J. Math. Phys. 5, 591 (1964).

[44] J. Schwinger, J. Math. Phys. 5, 1606 (1964).

[45] T. Teubner, diploma thesis, University of Karlsruhe, 1992.

[46] R. Harlander, diploma thesis, University of Karlsruhe, 1995.

[47] A. S. Kronfeld, Phys. Rev. D 58, 051501 (1998).

[48] R. Tarrach, Nucl. Phys. B183, 384 (1981).

[49] M. Beneke and V. M. Braun, Nucl. Phys. B426, 301 (1994).

[50] I. I. Bigi et al., Phys. Rev. D 50, 2234 (1994).

[51] M. C. Smith and S. S. Willenbrock, Phys. Rev. Lett. 79, 3825 (1997).

[52] A. H. Hoang, M. C. Smith, T. Stelzer, and S. S. Willenbrock, Phys. Rev. D 59, 114014 (1999).

[53] M. Beneke, Phys. Lett. B 434, 115 (1998).

[54] U. Aglietti and Z. Ligeti, Phys. Lett. B 364, 75 (1995).

[55] M. Beneke and V. M. Braun, Phys. Lett. B 348, 513 (1995).

[56] K. Melnikov and A. Yelkhovsky, Phys. Rev. D 59, 114009 (1999).

[57] A. Pineda and F. J. Yndurain, Phys. Rev. D 58, 094022 (1998).

[58] T. Appelquist, M. Dine, and I. J. Muzinich, Phys. Rev. D 17, 2074 (1978).

[59] N. Brambilla, A. Pineda, J. Soto, and A. Vairo, hep-ph/9903355.

[60] A. H. Hoang, Z. Ligeti, and A. V. Manohar, Phys. Rev. Lett. 82, 277 (1999).

[61] A. H. Hoang, Z. Ligeti, and A. V. Manohar, Phys. Rev. D 59, 074017 (1999). 
[62] K. G. Chetyrkin, J. H. Kühn, and M. Steinhauser, Phys. Lett. B 351, 331 (1995).

[63] A. H. Hoang, J. H. Kühn, and T. Teubner, Nucl. Phys. B452, 173 (1995).

[64] M. B. Voloshin, Phys. Rev. D 46, 3062 (1992).

[65] I. Bigi, M. Shifman, N. Uraltsev, and A. Vainshtein, Phys. Rev. D 56, 4017 (1997).

[66] S. J. Brodsky and Hung Jung Lu, Phys. Rev. D 51, 3652 (1995).

[67] S. J. Brodsky, G. P. Lepage, and P. B. Mackenzie, Phys. Rev. D 28, 228 (1983).

[68] K. G. Chetyrkin, A. H. Hoang, J. H. Kühn, M. Steinhauser, and T. Teubner, Eur. Phys. J. C 2, 137 (1998).
[69] M. Beneke, A. Signer, and V. A. Smirnov, Phys. Lett. B 454, 137 (1999)

[70] T. Nagano, A. Ota, and Y. Sumino, Phys. Rev. D (to be published), hep-ph/9903498.

[71] A. A. Penin and A. A. Pivovarov, hep-ph/9904278.

[72] G. Källen and A. Sabry, K. Dan. Vidensk. Selsk. Mat. Fys. Medd. 29, 1 (1955).

[73] A. H. Hoang, Phys. Rev. D 56, 7276 (1997).

[74] M. B. Voloshin, Int. J. Mod. Phys. A 10, 2865 (1995).

[75] S. G. Karshenboim, Yad. Fiz. 56, 155 (1993) [Phys. At. Nucl. 56, 1710 (1993)].

[76] K. G. Chetyrkin, J. H. Kühn, and M. Steinhauser, Nucl. Phys. B482, 213 (1996). 